\title{
On the Cohomology of Locally Symmetric Spaces and of their Compactifications
}

\author{
Leslie Saper
}

\begin{abstract}
This expository article gives an introduction to the (generalized) conjecture of Rapoport and Goresky-MacPherson which identifies the intersection cohomology of a real equal-rank Satake compactification of a locally symmetric space with that of the reductive Borel-Serre compactification. We motivate the conjecture with examples and then give an introduction to the various topics that are involved: intersection cohomology, the derived category, and compactifications of a locally symmetric space, particularly those above. We then give an overview of the theory of $\mathcal{L}$-modules and micro-support which was developed to solve the conjecture but has other important applications as well. We end with sketches of the proofs of three main theorems on $\mathcal{L}$-modules that lead to the resolution of the conjecture. The text is enriched with many examples, illustrations, and references to the literature.
\end{abstract}

\section{CONTENTS}

1. An example 220

2. $L^{2}$-cohomology 221

3. Zucker's conjecture 223

4. Intersection cohomology 224

5. Compactifications of locally symmetric spaces 228

6. The Borel-Serre compactification 231

7. The reductive Borel-Serre compactification 236

8. Satake compactifications 238

9. The Rapoport/Goresky-MacPherson conjecture 243

10. Weighted cohomology 243

11. Main theorem 245

12. L-modules 245

13. Micro-support of an $\mathcal{L}$-module 254

14. A vanishing theorem for the cohomology of an $\mathcal{L}$-module 257

An expanded version of two talks at the Harvard-M.I.T. conference "Current Developments in Mathematics, 2002", November 15-17.

This material is based upon work supported in part by the National Science Foundation under grant DMS-9870162. The manuscript was typeset using AMS-IATEX; the XY-pic package was used for figures and diagrams. 
15. Application: a vanishing theorem for ordinary cohomology 258

16. A vanishing theorem for $L^{2}$-cohomology 260

17. Proof of the vanishing theorem for the cohomology of an $\mathcal{L}$-module 260

18. Micro-support of intersection cohomology 269

19. Functoriality of micro-support 280

20. Proof of the Rapoport/Goresky-MacPherson conjecture 285

21. A generalization of Goresky-Harder-MacPherson's theorem 286

References

\section{An example}

We begin with the simplest example of a locally symmetric space. This is the moduli of complex elliptic curves $\mathbb{C} / \Lambda$, where $\Lambda$ is a lattice in $\mathbb{C}$. The moduli space can be expressed as

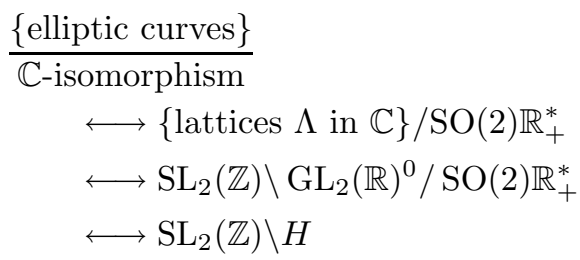

where $H=\{z \in \mathbb{C} \mid \operatorname{Im} z>0\}$ is the upper half-plane and $\mathbb{R}_{+}^{*}$ denotes the group of positive real scalar matrices. Here we let $\left(\begin{array}{ll}a & b \\ c & d\end{array}\right) \in \mathrm{GL}_{2}(\mathbb{R})^{0}$ parametrize the lattice $\Lambda=\mathbb{Z}(a+i b)+\mathbb{Z}(c+i d)$; the quotient by $\mathrm{SL}_{2}(\mathbb{Z})$ removes the dependence on the choice of an oriented basis. For future purposes, we will actually view $H$ as $\mathrm{GL}_{2}(\mathbb{R}) / \mathrm{O}(2) \mathbb{R}_{+}^{*}$.

A fundamental domain for the action of $\mathrm{SL}_{2}(\mathbb{Z})$ is pictured in Figure 1; the action of $\mathrm{SL}_{2}(\mathbb{Z})$ further identifies $z \leftrightarrow-\bar{z}$ for points in the boundary of the domain. Note the "cusp" at infinity which is not properly part of $X=\mathrm{SL}_{2}(\mathbb{Z}) \backslash H$ but may be added to yield a compactification $X^{*}$. One may also consider $X=\Gamma \backslash H$ where $\Gamma$ is a finite-index subgroup of $\mathrm{SL}_{2}(\mathbb{Z})$. Then $X^{*}$ is formed by adding possibly several cusp points. Any representation $(\sigma, E)$ of $\mathrm{GL}_{2}(\mathbb{R})$ defines a locally constant sheaf $\mathbb{E}$ on $X$, namely $\Gamma \backslash(H \times E)=H \times E / \sim$, where $(x, v) \sim(\gamma x, \sigma(\gamma) v)$ for all $\gamma \in \Gamma$. The classical Eichler-Shimura theorem [71] states

THEOREM 1.1. Let $E_{k}$ be the representation of $\mathrm{GL}_{2}(\mathbb{R})$ with highest weight $k$. Then $H_{P}^{1}\left(X^{*} ; \mathbb{E}_{k}\right) \cong \mathcal{S}_{k+2}(\Gamma) \oplus \overline{\mathcal{S}_{k+2}(\Gamma)}$.

The group appearing on the left-hand side is the parabolic cohomology:

$$
H_{P}^{1}\left(X^{*} ; \mathbb{E}_{k}\right) \equiv \operatorname{Ker}\left(H^{1}\left(X ; \mathbb{E}_{k}\right) \rightarrow \bigoplus_{x \in \operatorname{cusps}} H^{1}\left(U_{x} ; \mathbb{E}_{k}\right)\right)
$$

where $U_{x}$ is a punctured neighborhood of the cusp point $x$. It may also be defined by a modification of the usual complex computing the group cohomology $H\left(\Gamma ; E_{k}\right)$. One should think of parabolic cohomology as a modification of ordinary cohomology in which the local cohomology near a cusp in degree 1 has been killed or truncated. 


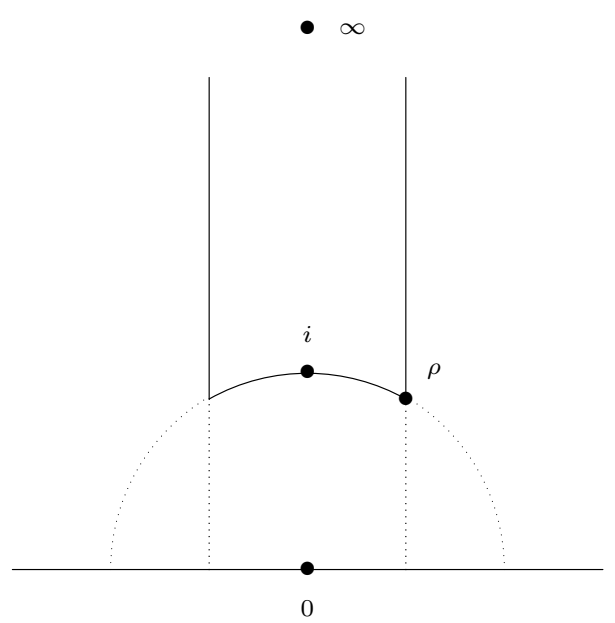

Figure 1. The fundamental domain for the action of $\mathrm{SL}_{2}(\mathbb{Z})$ on the upper half-plane

The space $S_{k+2}(\Gamma)$ on the right-hand side is the space of modular cusp forms of weight $k+2$ for $\Gamma$ :

$$
f \in \mathcal{S}_{k+2}(\Gamma) \Longleftrightarrow\left\{\begin{array}{l}
f: H \rightarrow \mathbb{C} \text { holomorphic, } \\
f\left(\frac{a z+b}{c z+d}\right)=(c z+d)^{k+2} f(z) \text { for }\left(\begin{array}{ll}
a & b \\
c & d
\end{array}\right) \in \Gamma, \\
f \text { vanishes at the cusps. }
\end{array}\right.
$$

Modular forms (and their generalization, automorphic forms) play a central role in number theory. For example, note that the space $X^{*}$ is actually an algebraic variety defined over a number field. An important application of Theorem 1.1 is that, for $\Gamma$ a congruence subgroup, it allows one to relate the Hasse-Weil zeta function of $X^{*}$ (which encodes the number of points of $X^{*}$ defined over all finite fields) to the $L$-functions associated to modular forms. We will not discuss this further here; for details see Shimura's book [71].

\section{2. $L^{2}$-cohomology}

We now interpolate a group within the Eichler-Shimura isomorphism:

$$
H_{P}^{1}\left(X^{*} ; E_{k}\right) \cong H_{(2)}^{1}\left(X ; \mathbb{E}_{k}\right) \cong \mathcal{S}_{k+2}(\Gamma) \oplus \overline{\mathcal{S}_{k+2}(\Gamma)}
$$

The new group $H_{(2)}\left(X ; \mathbb{E}_{k}\right)$ is the $L^{2}$-cohomology.

The $L^{2}$-cohomology is a variant of de Rham cohomology formed by imposing an $L^{2}$-growth condition on the differential forms. We will briefly review the theory in a general context; good references are the papers of Cheeger [26], Cheeger, Goresky, and MacPherson [27], and Zucker [81]. Suppose that $M$ is an oriented Riemannian manifold (a $V$-manifold [66] or orbifold is sufficient) and that $\mathbb{E}$ is a metrized locally constant sheaf. In other words, $\mathbb{E}$ is the sheaf of sections of a flat vector bundle on $M$ equipped with a fiber-wise Hermitian metric (which is not necessarily flat). Let $d$ denote the exterior derivative on $A(M ; \mathbb{E})$, the smooth $\mathbb{E}$-valued differential 
forms on $M$. For every $x \in M$, the norm on the tangent space $T_{x} M$ and the norm on the fiber $\mathbb{E}_{x}$ induce a norm on $\bigwedge T_{x}^{*} M \otimes \mathbb{E}_{x}$. Thus for $\phi \in A(M ; \mathbb{E})$, we have a function $x \mapsto|\phi(x)|$. On the other hand, the Riemannian metric also induces a volume form $d V$. We define the $L^{2}$-norm of $\phi$ by

$$
\|\phi\|^{2} \equiv \int_{M}|\phi|^{2} d V
$$

and set

$$
A_{(2)}(M ; \mathbb{E}) \equiv\{\phi \in A(M ; \mathbb{E}) \mid\|\phi\|<\infty \text { and }\|d \phi\|<\infty\} .
$$

The $L^{2}$-cohomology $H_{(2)}(M ; \mathbb{E})$ is defined to be the cohomology of the complex $\left(A_{(2)}(M ; \mathbb{E}), d\right)$; it has a topology (which may not be Hausdorff) given by the seminorm

$$
\|[\phi]\| \equiv \inf _{\eta \in A_{(2)}(M ; \mathbb{E})}\|\phi+d \eta\| \quad \text { for }[\phi] \in H_{(2)}(M ; \mathbb{E}) .
$$

The $L^{2}$-cohomology is a quasi-isometry invariant of the metrics on $M$ and $\mathbb{E}$ : two metrics $h$ and $h^{\prime}$ are said to be quasi-isometric if $c^{-1} h \leq h^{\prime} \leq c h$ uniformly for some $c>0$.

Sometimes it is useful to consider forms which are not necessarily smooth, but only measurable. The measurable forms $\phi$ which are $L^{2}$ and which have exterior derivatives in the distribution sense represented by $L^{2}$ forms yield a complex which we denote $(\operatorname{Dom} \bar{d}, \bar{d})$; this is indeed the closure in the sense of unbounded operators on Hilbert space of the $d$ operator acting on the domain $A_{(2)}(M, \mathbb{E})$. A smoothing argument $[\mathbf{2 6}],[\mathbf{2 8}]$ shows that this extended complex computes the same cohomology,

$$
H_{(2)}(M ; \mathbb{E})=H(\operatorname{Dom} \bar{d})=\operatorname{Ker} \bar{d} / \operatorname{Im} \bar{d} .
$$

It follows that

$$
\begin{aligned}
H_{(2)}(M ; \mathbb{E}) & =(\operatorname{Ker} \bar{d} / \overline{\operatorname{Im} \bar{d}}) \oplus(\overline{\operatorname{Im} \bar{d}} / \operatorname{Im} \bar{d}) \\
& \cong\left(\operatorname{Ker} \bar{d} \cap \operatorname{Ker} \bar{d}^{*}\right) \oplus(\overline{\operatorname{Im} \bar{d}} / \operatorname{Im} \bar{d}) .
\end{aligned}
$$

This is Hodge theory: the first term is a certain space of $L^{2}$ harmonic forms (in fact all $L^{2}$ harmonic forms if the Riemannian metric is complete) and the second term is either 0 or infinite dimensional. Note that $H_{(2)}(M ; \mathbb{E})$ is Hausdorff if and only if the second term is 0 .

In the example of $\S 1$, we give $H$ the hyperbolic metric $\left(d x^{2}+d y^{2}\right) / y^{2}$ under which $H$ becomes a symmetric space. Since the metric is invariant under the action of $\mathrm{SL}_{2}(\mathbb{Z})$, it descends to a metric on $X=\Gamma \backslash H$, a locally symmetric space. Furthermore, any finite dimensional representation $(\sigma, E)$ of $\mathrm{GL}_{2}(\mathbb{R})$ admits an admissible inner product, that is, one for which $\sigma(g)^{*}=\sigma(\theta g)^{-1}$, where $\theta$ denotes the Cartan involution $g \mapsto{ }^{t} g^{-1}$. Such an inner product induces a Hermitian metric on $\mathbb{E}=\Gamma \backslash(H \times E)$, namely

$$
\|(g \mathrm{SO}(2), v)\|=\left\|\sigma\left(g^{-1}\right) v\right\| \quad \text { for } g \in \mathrm{SL}_{2}(\mathbb{R}) .
$$

A variant of this definition applies to $g \in \mathrm{GL}_{2}(\mathbb{R})$. These metrics on $X$ and $\mathbb{E}$ are unique up to quasi-isometry (in fact up to scalar multiples if $E$ is irreducible) and thus the $L^{2}$-cohomology $H_{(2)}\left(X ; \mathbb{E}_{k}\right)$ is well-defined. 
The first isomorphism of (1) is heuristically true since the metric near a cusp, say the cusp at $\infty$, may be expressed as

$$
d r^{2}+e^{-2 r} d \theta^{2}, \quad \text { where } r=\log y \in[b, \infty) \text { and } \theta=x \text { modulo } \mathbb{Z},
$$

and hence $d \theta$ is not $L^{2}$. The second isomorphism of (1) follows from Hodge theory and the Hodge decomposition: the coefficients of harmonic forms representing type $(1,0)$ cohomology classes can easily be seen to be modular forms of the appropriate weight; the $L^{2}$ condition implies the form is cuspidal.

Other moduli problems yield locally symmetric spaces and in view of the application mentioned in $\S 1$ and others, one would like a generalization of (1). In this paper, we will focus on the first isomorphism of (1).

\section{Zucker's conjecture}

In general a locally symmetric space has the form

$$
X=\Gamma \backslash G / K A_{G}=\Gamma \backslash D
$$

where

$$
\begin{aligned}
& G \equiv \text { the real points of a connected reductive algebraic } \\
& \text { group defined over } \mathbb{Q}, \\
& K \equiv \text { a maximal compact subgroup of } G \\
& A_{G} \equiv \text { the identity component }\left(\mathbb{R}_{+}^{*}\right)^{s} \text { of a maximal } \mathbb{Q} \text { - } \\
& \quad \text { split torus in the center of } G, \\
& D \equiv G / K A_{G}, \text { the corresponding symmetric space, } \\
& \Gamma \equiv \text { an arithmetic subgroup of } G .
\end{aligned}
$$

We always give $D$ a $G$-invariant Riemannian metric; this induces a Riemannian metric on $X$. Although the locally symmetric space $X$ may have finite quotient singularities, for simplicity we will treat it and related spaces as if they were smooth; the correct treatment involves the language of $V$-manifolds or orbifolds. As in the case of $\mathrm{GL}_{2}(\mathbb{R})$, any representation $E$ of $G$ induces a metrized locally constant sheaf $\mathbb{E}$ on $X$.

Notation. In this paper we will indicate Lie groups, often defined as the real points of an algebraic group, by uppercase roman letters; their Lie algebras will be denoted by the corresponding lowercase fraktur letter. In order to lighten the exposition and the notation, we will take some liberties in terminology in this paper. Specifically if $H$ is the Lie group of the real points (or the identity component of the real points) of an algebraic group defined over $\mathbb{Q}$, we may refer to $H$ when properly we should refer to the underlying algebraic group. For example we might speak of a rational character of $H$ defined over $\mathbb{Q}$. Or we will speak of a parabolic $\mathbb{Q}$-subgroup $P$ of $H$, when we properly should be referring to the real points of a parabolic $\mathbb{Q}$-subgroup of the algebraic group underlying $H$.

An important special case is a Hermitian locally symmetric space, where $D$ has a $G$-invariant complex structure. The generalization of the first isomorphism in (1) is

Theorem 3.1 (Zucker's Conjecture). Let $X$ be a Hermitian locally symmetric space. There is a natural isomorphism $I_{p} H\left(X^{*} ; \mathbb{E}\right) \cong H_{(2)}(X ; \mathbb{E})$, where $I_{p} H$ denotes middle-perversity intersection cohomology. 
This result was first conjectured by Zucker in [81] where some simple cases were proven. Other special cases of this result were proven by Borel [10], Borel and Casselman [14], and Zucker [83]. The theorem in general was proved by Looijenga [49] and independently by the author and Stern [65].

There are two new ingredients in this theorem we need to explain: the middleperversity intersection cohomology $I_{p} H\left(X^{*} ; \mathbb{E}\right)$, which plays the role of parabolic cohomology, and the Baily-Borel-Satake compactification $X^{*}$, which generalizes adjoining cusp points to $\Gamma \backslash H$. Along the way we will also introduce the BorelSerre compactification $\bar{X}$ and the reductive Borel-Serre compactification $\widehat{X}$ which will be important for the conjecture of Rapoport and Goresky-MacPherson.

\section{Intersection cohomology}

Intersection cohomology, developed by Goresky and MacPherson $[\mathbf{3 2}],[\mathbf{3 3}]$, is a cohomology theory for singular spaces $Z$ which shares many of the properties of ordinary cohomology theory applied to smooth spaces (e.g. Poincaré duality). It depends on a locally constant sheaf $\mathbb{E}$ on the smooth locus and a perversity:

$$
p:\{2, \ldots, d\} \rightarrow \mathbb{Z}, \quad p(2)=0, \quad p(k) \leq p(k+1) \leq p(k)+1 .
$$

We will mainly be interested in $p$ being one of the middle perversities:

$$
n(k)=\left\lfloor\frac{(k-1)}{2}\right\rfloor \quad \text { or } \quad m(k)=\left\lfloor\frac{(k-2)}{2}\right\rfloor ;
$$

this is what is required in Zucker's conjecture and later in the conjecture of Rapoport and Goresky-MacPherson. Besides the original papers, a useful reference is Borel's book [15].

4.1. Simplicial intersection cohomology. The type of singular space for which $I_{p} H(Z ; \mathbb{E})$ is usually defined is a pseudomanifold (see [37], [43], and [55] for more general contexts). A d-dimensional stratified pseudomanifold $Z$ is a topological space with a filtration by closed subsets

$$
Z=Z_{d} \supset Z_{d-1}=Z_{d-2} \supset Z_{d-3} \supset \cdots \supset Z_{0} \supset Z_{-1}=\emptyset
$$

such that

(i) For all $k \geq 0$, the subspace $S^{k}=Z_{d-k} \backslash Z_{d-k-1}$ is a topological $(d-k)$ manifold, called the codimension- $k$ stratum.

(ii) The stratum $S^{0}$ is dense.

(iii) Any point $x \in S^{k}$ has an open neighborhood $U$ in $Z$ which is homeomorphic (as a stratified pseudomanifold) to $B_{d-k} \times c\left(L_{k-1}\right)$, where $B_{d-k}$ is a ball neighborhood of $x$ in $S^{k}$ and $c\left(L_{k-1}\right)$ is a cone on a $(k-1)$ dimensional stratified pseudomanifold called the link.

The coefficient system $\mathbb{E}$ is a locally constant sheaf defined on $S^{0}$, the nonsingular stratum of $Z$. Examples of pseudomanifolds include complex analytic varieties as well as the reductive Borel-Serre compactification and the Satake compactifications of locally symmetric spaces to be discussed later.

The first definition of intersection cohomology [32], however, used a piecewiselinear structure on $Z$. Briefly, assume that $Z$ is a piecewise-linear stratified pseudomanifold (that is, all spaces and maps in the above definition are taken in the piecewise-linear category). A locally finite geometric chain $\xi$ is a locally finite simplicial chain for some triangulation of $Z$, however we identify two chains if they 
agree after passing to a common refinement of the triangulations; let $|\xi|$ denote the support of the chain. Let $\mathcal{O}$ denote the orientation sheaf of $S^{0}$ and let $I_{p} C^{j}(Z ; \mathbb{E})$ denote the locally finite geometric $(d-j)$-chains $\xi$ with values in $\mathbb{E} \otimes \mathcal{O}$ that satisfy the allowability conditions

$$
\begin{aligned}
\operatorname{dim}\left(|\xi| \cap Z_{d-k}\right) & \leq d-j-k+p(k), \\
\operatorname{dim}\left(|\partial \xi| \cap Z_{d-k}\right) & \leq d-j-1-k+p(k),
\end{aligned}
$$

for all $k \geq 2$. Even though $\mathbb{E}$ is not defined on all of $Z$, the equations above for $k=2$ ensure that the interiors of the $(d-j)$-simplices and the $(d-j-1)$-simplices in $\xi$ lie in $S^{0}$, so it makes sense to say that $\xi$ has "values in $\mathbb{E} \otimes \mathcal{O}$ ". The role of the perversity $p(k)$ in these equations is to bound the amount of intersection allowed beyond that which would occur in the case of transversal intersection.

Under the usual boundary operator, $I_{p} C(Z ; \mathbb{E})$ becomes a complex whose cohomology is the intersection cohomology $I_{p} H(Z ; \mathbb{E})$. The key thing to keep in mind about intersection cohomology is its local characterization. If $U=B_{d-k} \times c\left(L_{k-1}\right)$ is a local neighborhood of $x \in S^{k}$ as above, then

$$
I_{p} H^{j}\left(B_{d-k} \times c\left(L_{k-1}\right) ; \mathbb{E}\right) \cong \begin{cases}I_{p} H^{j}\left(L_{k-1} ; \mathbb{E}\right) & \text { for } j \leq p(k), \\ 0 & \text { for } j>p(k) .\end{cases}
$$

Indeed this calculation and the facts that the Mayer-Vietoris sequence holds for intersection cohomology and that intersection cohomology agrees with ordinary cohomology on a manifold, suffice to inductively determine $I_{p} H(Z ; \mathbb{E})$. Since $m(k)=$ $n(k)$ for $k$ even, it follows that both $p=m$ and $p=n$ yield the same theory for $Z$ with only even codimension strata (for example, a complex analytic variety); certain spaces with possibly odd codimension strata will become important later. In our example of a quotient of the upper half-plane, a cusp $x$ has codimension 2 and so the local intersection cohomology at $x$ vanishes in degrees $j>p(2)=0$ for $p=m$ or $n$; it is easy to see then that $I_{p} H^{1}\left(X^{*} ; \mathbb{E}_{k}\right) \cong H_{P}^{1}\left(X^{*} ; \mathbb{E}_{k}\right)$.

The constraints on a ordinary perversity and the condition in the definition of a stratified pseudomanifold that $S^{1}=\emptyset$ ensure that $I_{p} H(Z ; \mathbb{E})$ is independent of the stratification; it is not apparent however with this combinatorial definition that intersection cohomology is independent of the piecewise-linear structure.

4.2. The derived category. It has proven useful to take a sheaf-theoretic approach to intersection cohomology [33]; in particular, this allows an easy proof of topological invariance. In this approach, intersection cohomology is the hypercohomology of a certain object $\mathcal{I}_{p} \mathcal{C}(Z ; \mathbb{E})$ in the constructible derived category of sheaves. We begin by briefly describing this category; a good reference is the book of Kashiwara and Schapira [42].

The (bounded) derived category of sheaves $\mathbf{D}^{b}(Z)$ has as objects bounded complexes of sheaves

$$
\mathcal{S} \equiv \quad \cdots \stackrel{d_{n-2}}{\longrightarrow} \mathcal{S}^{n-1} \stackrel{d_{n-1}}{\longrightarrow} \mathcal{S}^{n} \stackrel{d_{n}}{\longrightarrow} \cdots
$$

on $Z$. A single sheaf will always be treated as a complex which is zero except in degree 0 . The definition of morphism is more subtle. Before giving it, recall that the cohomology sheaf $H(\mathcal{S})$ of $\mathcal{S}$ is the complex of sheaves (with zero differential) defined by $H^{n}(\mathcal{S}) \equiv \operatorname{Ker} d_{n} / \operatorname{Im} d_{n-1}$. Any morphism $\psi: \mathcal{S} \rightarrow \mathcal{S}^{\prime}$ between complexes of sheaves induces a morphism $H(\psi): H(\mathcal{S}) \rightarrow H\left(\mathcal{S}^{\prime}\right)$ between the cohomology 
sheaves; $\psi$ is called a quasi-isomorphism if $H(\psi)$ is an isomorphism. Finally a morphism $\phi \in \operatorname{Mor}_{\mathbf{D}^{b}(Z)}$ is a certain equivalence class of diagrams $\mathcal{S} \stackrel{\phi_{1}}{\longleftarrow} \mathcal{S}^{\prime \prime} \stackrel{\phi_{2}}{\longrightarrow} \mathcal{S}^{\prime}$ of sheaf complex morphisms such that $\phi_{1}$ is a quasi-isomorphism. (We will not define the equivalence relation here; to begin with, $\phi_{1}$ and $\phi_{2}$ should be taken to be homotopy classes of sheaf complex morphisms.) One defines a notion of composition of such morphisms.

It is clear that any sheaf complex morphism $\psi$ induces a morphism of the corresponding objects in the derived category of sheaves; one sets $\mathcal{S}^{\prime \prime}=\mathcal{S}$ with $\phi_{1}$ the identity and $\phi_{2}=\psi$. The definition is arranged so that a quasi-isomorphism becomes an isomorphism in the derived category and in fact we say that two complexes of sheaves are quasi-isomorphic if they are isomorphic in the derived category. Loosely put, the derived category is a localization of the ordinary category of complexes of sheaves obtained by "inverting" the quasi-isomorphisms. The cohomology sheaf $H(\mathcal{S})$ is well-defined for an object in the derived category and the constructible derived category is the full subcategory consisting of objects $\mathcal{S}$ such that $\left.H(\mathcal{S})\right|_{S^{k}}$ is a locally constant sheaf with finitely generated stalks for every stratum $S^{k}$.

4.3. Functors on the derived category. Any bounded complex of sheaves $\mathcal{S}$ has an injective resolution: a quasi-isomorphism $\mathcal{S} \rightarrow \mathcal{I}$ where $\mathcal{I}$ is a complex of injective sheaves; this is unique up to isomorphism in the derived category. Thus for any left exact functor $F$ on sheaves one may define a corresponding functor $R F$ (the right derived functor) on the derived category of sheaves:

$$
R F(\mathcal{S}) \equiv F(\mathcal{I})
$$

We will write $R F$ simply as $F$ since we will never consider the original functor $F$ on complexes of sheaves. In this way, for any continuous map $k: Z \rightarrow W$ we obtain functors $k_{*}, k_{!}$, and $k^{*}$ on the derived category. (The functor $k^{*}$ is actually exact so one does not even need to pass to an injective resolution.) If $k$ is a stratified map $[33, \S 1.2]$ these are in fact functors on the constructible derived category.

The hypercohomology of an object $\mathcal{S}$ of the derived category is the right derived functor of the global sections functor $\Gamma(Z ; \cdot)$. Thus $H(Z ; \mathcal{S}) \equiv H(\Gamma(Z ; \mathcal{I}))$.

The functor $k^{*}$ is a left adjoint to $k_{*}$ :

$$
\operatorname{Mor}_{\mathbf{D}^{b}(Z)}\left(k^{*} \mathcal{S}, \mathcal{S}^{\prime}\right)=\operatorname{Mor}_{\mathbf{D}^{b}(W)}\left(\mathcal{S}, k_{*} \mathcal{S}^{\prime}\right) .
$$

It follows that there is a natural adjunction morphism

$$
\mathcal{S} \longrightarrow k_{*} k^{*} \mathcal{S}
$$

characterized by the condition that $k^{*} \mathcal{S} \longrightarrow k^{*} k_{*} k^{*} \mathcal{S}=k^{*} \mathcal{S}$ is the identity morphism.

With slightly more work, one may also define a functor $k^{!}$on the derived category which is a right adjoint to $k_{\text {! }}$. We will only need $k^{!}$when $k$ is the inclusion of a locally closed subset; in this case $k^{!}=k^{*} \circ R \Gamma_{Z}$, the derived "sections supported by $Z$ " functor. Concretely, $k^{!}(\mathcal{S})$ is the sheaf defined by

$$
k^{!}(\mathcal{S})(U) \equiv \operatorname{Ker}(\Gamma(\widetilde{U} ; \mathcal{I}) \rightarrow \Gamma(\widetilde{U} \backslash(Z \cap \widetilde{U}) ; \mathcal{I})),
$$

where $\widetilde{U} \subseteq W$ is an open subset such that $U=Z \cap \widetilde{U}$ is closed in $\widetilde{U}$.

It is not always necessary to use injective resolutions. For example, for hypercohomology it suffices to use a resolution by a complex of soft or fine sheaves provided the space is paracompact (as we will assume). 
4.4. Sheaf-theoretic intersection cohomology. We need one more functor on the derived category. This is the truncation functor $\tau^{\leqslant n}$ defined by

$$
\tau^{\leqslant n} \mathcal{S} \equiv \quad \cdots \stackrel{d_{n-2}}{\longrightarrow} \mathcal{S}^{n-1} \stackrel{d_{n-1}}{\longrightarrow} \operatorname{ker} d_{n} \stackrel{d_{n}}{\longrightarrow} 0 \longrightarrow \cdots
$$

it is characterized by the fact there is a natural morphism $\tau^{\leqslant n} \mathcal{S} \rightarrow \mathcal{S}$ which induces an isomorphism on cohomology sheaves in degrees $\leq n$ and the zero map on cohomology sheaves in degrees $>n$.

For all $k \geq 2$, let $U_{k} \equiv Z \backslash Z_{d-k}$, and let $j_{k}: U_{k} \hookrightarrow U_{k+1}=U_{k} \cup S^{k}$ and $i_{k}: S^{k} \hookrightarrow U_{k+1}$ be the inclusions. (We will find it convenient in situations like this to use the same symbols to denote the inclusions of the same sets into larger subspaces.) The local calculation (8) is encoded into an object of the constructible derived category by Deligne's formula $[\mathbf{7}],[\mathbf{3 3}]$ :

$$
\mathcal{I}_{p} \mathcal{C}(Z ; \mathbb{E}) \equiv \tau^{\leqslant p(d)} j_{d *} \cdots \tau^{\leqslant p(3)} j_{3 *} \tau^{\leqslant p(2)} j_{2 *} \mathbb{E} .
$$

This object is characterized up to isomorphism in the derived category by the following conditions:

(i) $i_{2}^{*} \mathcal{S} \cong \mathbb{E}$.

(ii) $H^{j}\left(i_{k}^{*} \mathcal{S}\right)=0$ for $j>p(k)$.

(iii) The attaching morphism $i_{k}^{*} \mathcal{S} \rightarrow i_{k}^{*} j_{k *} j_{k}^{*} \mathcal{S}$ induces an isomorphism on cohomology sheaves in degrees $\leq p(k)$.

By definition, the intersection cohomology $I_{p} H(Z ; \mathbb{E})$ is the hypercohomology of $\mathcal{I}_{p} \mathcal{C}(Z ; \mathbb{E})$. It is possible to recast these conditions in an equivalent form that does not make explicit use of the stratification $[\mathbf{1 5}],[\mathbf{3 3}]$; this proves that intersection cohomology is a topological invariant.

In the piecewise-linear case, the allowable locally finite geometric chains define a complex of sheaves $U \mapsto I_{p} C(U ; \mathbb{E})$ which satisfies the above conditions (essentially by (8)) and hence is quasi-isomorphic to $\mathcal{I}_{p} \mathcal{C}(Z ; \mathbb{E})$. These sheaves are also soft, so their hypercohomology may be computed as the cohomology of global sections; it follows that the two definitions of intersection cohomology agree.

As in [7], we will sometimes consider a more general notion of perversity which is simply an integer-valued function $p: \mathcal{S} \rightarrow \mathbb{Z}$ on the set of strata $\mathcal{S}$. The formula (9) may be generalized to such $p$ and the corresponding intersection cohomology may be defined; in general such a cohomology group depends upon the stratification.

4.5. Sheaf-theoretic $\boldsymbol{L}^{2}$-cohomology. If the nonsingular stratum $S^{0}$ of $Z$ has a Riemannian metric, one may localize the $L^{2}$-cohomology of $S^{0}$ at points of $Z$ to define the $L^{2}$-cohomology sheaf $\mathcal{L}_{(2)}(Z ; \mathbb{E})$. This is the complex of sheaves defined by the presheaf

$$
U \mapsto A_{(2)}\left(U \cap S^{0} ; \mathbb{E}\right)
$$

In general this sheaf may not be fine; the difficulty is finding a partition of unity for a cover of $Z$ with functions $\eta$ such that $\left.\eta\right|_{S^{0}}$ has bounded differential. However when this sheaf is fine, the $L^{2}$-cohomology of $S^{0}$ may be expressed as a hypercohomology group on $Z: H\left(Z ; \mathcal{L}_{(2)}(Z ; \mathbb{E})\right)=H_{(2)}\left(S^{0} ; \mathbb{E}\right)$.

Clearly the $L^{2}$-cohomology sheaf on the compactification $X^{*}$ of $\mathrm{SL}_{2}(\mathbb{Z}) \backslash H$ considered in $\S 1$ is fine. If instead one considers the compactification $\bar{X}$ obtained by adjoining $S^{1}=\mathbb{Z} \backslash \mathbb{R}$ (an example of the Borel-Serre compactification to be considered later), the sheaf would not be fine since $|d \theta| \rightarrow \infty$ near the cusp by (5). It is 
not difficult to show that $\mathcal{L}_{(2)}(Z ; \mathbb{E})$ is fine $[\mathbf{8 1},(4.4)]$, [83] when $Z$ is the reductive Borel-Serre compactification $\widehat{X}$ or one of the Satake compactifications $X^{*}$ of a locally symmetric space to be introduced later.

The more precise form of Zucker's conjecture and Theorem 3.1 is that there is an isomorphism $\mathcal{I}_{p} \mathcal{C}\left(X^{*} ; \mathbb{E}\right) \cong \mathcal{L}_{(2)}\left(X^{*} ; \mathbb{E}\right)$ in the derived category.

\section{Compactifications of locally symmetric spaces}

We need to consider three compactifications of a locally symmetric space $X$ which fit into a diagram like this:

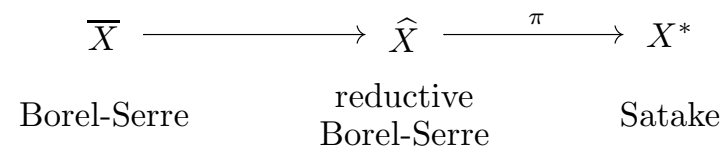

- The Borel-Serre compactification $\bar{X}[\mathbf{1 6}]$ is "topological": it corresponds to adding a boundary (or more generally corners) at infinity and does not change the homotopy type of $X$.

- The reductive Borel-Serre compactification $\widehat{X}$ (first constructed by Zucker in $[\mathbf{8 1},(4.1)])$ is "geometric": the boundary strata of $\bar{X}$ become collapsed so that the Riemannian metric on $X$ extends to a nondegenerate metric on each stratum at infinity.

- The compactification $X^{*}$ is one of the various Satake compactifications $[\mathbf{6 7}],[\mathbf{6 8}]$. Zucker $[\mathbf{8 2}]$ has shown that it may be viewed as a quotient of $\widehat{X}$. In the special case where $X$ is Hermitian locally symmetric, one of these is the Baily-Borel-Satake compactification. This is a complexanalytic compactification: the strata at infinity are further collapsed so that they also become Hermitian locally symmetric. In fact it is also an algebraic compactification: Baily and Borel [4] show in this case that $X^{*}$ is a projective algebraic variety defined over a number field.

We will give the constructions of the three compactifications carefully in $\S \S 6-8$ together with examples. In view of their importance to intersection cohomology, we will highlight for each compactification the boundary strata and their links. We will not however discuss the toroidal compactifications $\widetilde{X}[\mathbf{3}]$; these are defined in the Hermitian case and map to the Baily-Borel-Satake compactification $X^{*}$.

In the remainder of this section, we will describe the three compactifications qualitatively. First we give two simple examples.

5.1. Example: $\boldsymbol{X}=\mathrm{SL}_{2}(\mathbb{Z}) \backslash \boldsymbol{H}$. In this case there is only one non-proper stratum for all three compactifications and the situation is quite simple. A basic result of reduction theory shows that for $y \gg 0$, the identifications induced by $\mathrm{SL}_{2}(\mathbb{Z})$ are already induced by its parabolic subgroup $\left\{\left(\begin{array}{ll}1 & n \\ 0 & 1\end{array}\right) \mid n \in \mathbb{Z}\right\}$. This group preserves the lines $y=b$ and acts on them by translation by elements of $\mathbb{Z}$. Thus in $X$ for sufficiently large $y$ these lines wrap up into a family of circles; for the compactification $\bar{X}$ we adjoin yet another circle at $y=\infty$; see Figure 2. However the norm of the tangent vector to the circles in this family decays as $y \rightarrow \infty$ : $\left|\frac{\partial}{\partial x}\right|=\frac{1}{y} \rightarrow 0$. Thus the circle at infinity is collapsed to a point in $\widehat{X}$ and $X^{*}$. In summary: 


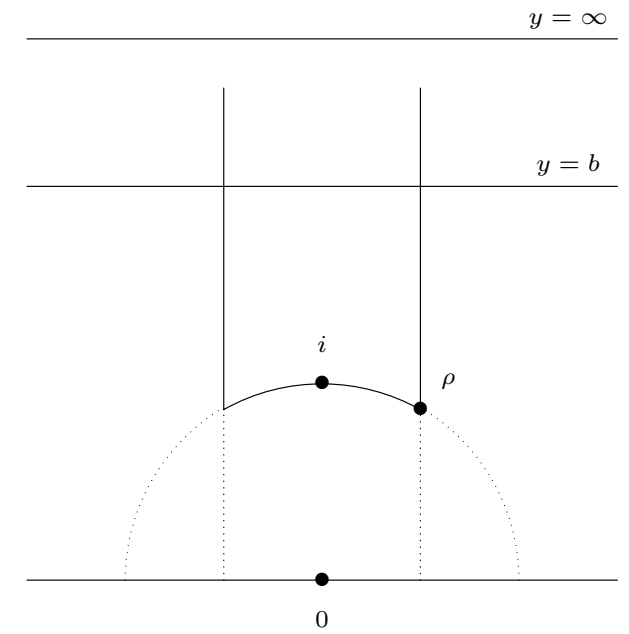

Figure 2. The upper half-plane with an additional line at $y=\infty$

\begin{tabular}{c|c|c} 
& Boundary stratum & Link \\
\hline $\bar{X}$ & $S^{1}$ & point \\
$\widehat{X}$ & point & $S^{1}$ \\
$X^{*}$ & point & $S^{1}$
\end{tabular}

5.2. Example: Hilbert modular surface. The simplest Hermitian example where all three compactifications are different is the Hilbert modular surface. (See $[\mathbf{3}, \S \mathrm{I} .5]$ for a nice short exposition and [39] for more details.) This is the moduli space of complex tori $\mathbb{C}^{2} / \Lambda$ with "real multiplication" by $\mathcal{O}_{k}$, the ring of integers of a real quadratic extension $k=\mathbb{Q}(\sqrt{d}), d>0$, of $\mathbb{Q}$. The symmetric space is simply $H \times H$; the arithmetic group $\Gamma=\mathrm{SL}_{2}\left(\mathcal{O}_{k}\right)$ acts on $H \times H$ via the embedding $\mathrm{SL}_{2}\left(\mathcal{O}_{k}\right) \hookrightarrow \mathrm{SL}_{2}(\mathbb{R}) \times \mathrm{SL}_{2}(\mathbb{R})$ given by the two real embeddings of $k$ in $\mathbb{R}$. Again, reduction theory shows that "near infinity" corresponding to $y_{1} y_{2} \gg 0$, the group $\Gamma=\mathrm{SL}_{2}\left(\mathcal{O}_{k}\right)$ acts via its parabolic subgroup

$$
\left\{\left(\begin{array}{cc}
u & 0 \\
0 & u^{-1}
\end{array}\right) \mid u \in \mathcal{O}_{k}^{\times}\right\} \ltimes\left\{\left(\begin{array}{ll}
1 & a \\
0 & 1
\end{array}\right) \mid a \in \mathcal{O}_{k}\right\}
$$

and there is only one non-proper stratum for all three compactifications.

Write $\mathcal{O}_{k}=\mathbb{Z}+\mathbb{Z} \delta$; the image of the map $\mathcal{O}_{k} \rightarrow \mathbb{R}^{2}$ given by the two real embeddings of $k$ in $\mathbb{R}$ is a lattice $\mathbb{Z}(1,1)+\mathbb{Z}(\delta, \bar{\delta})$. The second factor of (10) acts in the $x_{1} x_{2}$-plane via translation by this lattice. Let $u_{0}$ be a generator of the group of positive units $\mathcal{O}_{k+}^{\times}$. The first factor of (10) preserves the hyperbolas $y_{1} y_{2}=b$ and acts on them via $\left(y_{1}, y_{2}\right) \mapsto\left(u_{0}^{2 k}, u_{0}^{-2 k}\right)$ for all $k \in \mathbb{Z}$. Furthermore it effects an automorphism of the lattice in the $x_{1} x_{2}$-plane. Thus in $X$ for sufficiently large $y_{1} y_{2}$, we obtain a family of flat $T^{2}$-bundles (from the $x_{1} x_{2}$-plane) over $S^{1}$ 's (from the hyperbolas); for the compactification $\bar{X}$ we adjoin another copy of this bundle at $y_{1} y_{2}=\infty$; see Figure 3 .

To understand the non-proper stratum in the other two compactifications first note that the product metric on $H \times H$ may be rewritten as

$$
d r^{2}+d t^{2}+e^{-r}\left(e^{-t} d x_{1}^{2}+e^{t} d x_{2}^{2}\right) \quad \text { where } r=\log y_{1} y_{2} \text { and } t=\log y_{1} / y_{2} ;
$$



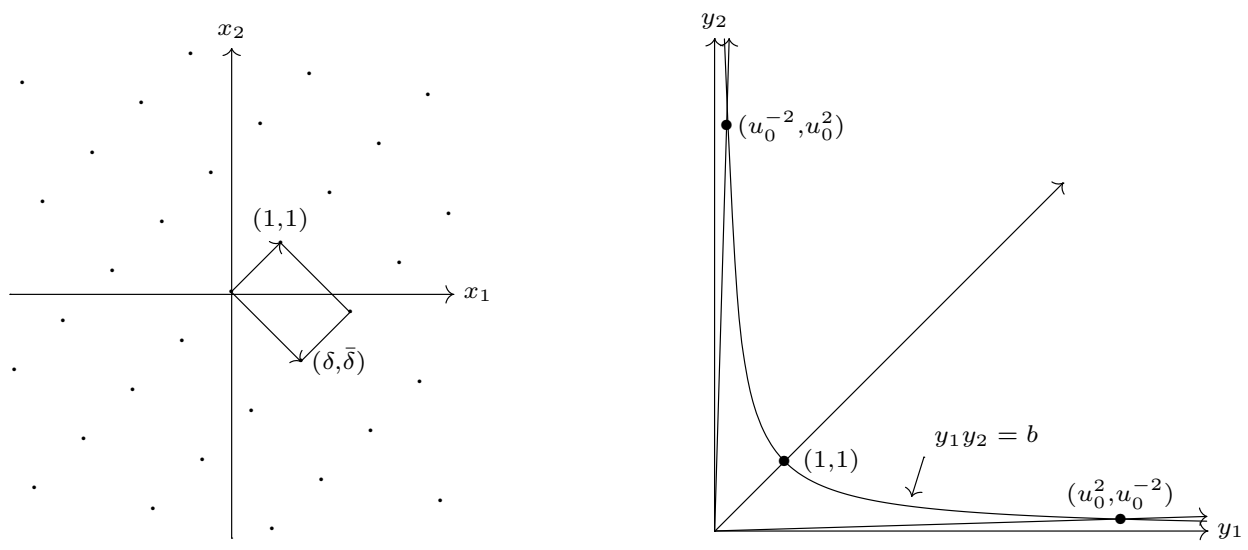

Figure 3. $\mathrm{SL}_{2}\left(\mathcal{O}_{k}\right)$ acting on $H \times H$

up to quasi-isometry this induces the metric

$$
d r^{2}+d s_{S^{1}}^{2}+e^{-r} d s_{T^{2}}^{2}
$$

on $X$ where $r \gg 0$. We see that the norm of a tangent vector to the $T^{2}$-fibers decays to 0 as $r \rightarrow \infty$ while the norm of a tangent vector to the base $S^{1}$ does not. Thus the $T^{2}$-fibers are collapsed in $\widehat{X}$ while the $S^{1}$ remains; since $S^{1}$ cannot have a complex structure, however, it is collapsed in $X^{*}$. In summary:

\begin{tabular}{c|c|c} 
& Boundary stratum & Link \\
\hline $\bar{X}$ & flat $T^{2}$-bundle over $S^{1}$ & point \\
$\widehat{X}$ & $S^{1}$ & $T^{2}$ \\
$X^{*}$ & point & flat $T^{2}$-bundle over $S^{1}$
\end{tabular}

5.3. The general case. In the general case the strata are no longer compact. For both the Borel-Serre compactification $\bar{X}$ and the reductive Borel-Serre compactification $\widehat{X}$ they are indexed by the set $\mathcal{P}$ of $\Gamma$-conjugacy classes of parabolic $\mathbb{Q}$-subgroups $P$ of $G$; for every $P \in \mathcal{P}$, we let $Y_{P}$ (respectively $X_{P}$ ) denote the corresponding stratum of $\bar{X}$ (respectively $\widehat{X}$ ). (Note that we will use the same symbol, say $P$, to denote both a parabolic $\mathbb{Q}$-subgroup and its $\Gamma$-conjugacy class.)

Associated to $G \in \mathcal{P}$ we have $X_{G}=Y_{G}=X$, the top-dimensional open stratum, while associated to a minimal parabolic $\mathbb{Q}$-subgroup $P_{0} \in \mathcal{P}$ is a minimaldimensional stratum. In fact the correspondence between the strata and $\mathcal{P}$ is order-preserving if we define the partial order on the set of strata by $S<T$ if and only if $S \subseteq \operatorname{cl}(T)$ (topological closure) and the partial order on $\mathcal{P}$ by $P<Q$ if and only if there exists $\gamma \in \Gamma$ so that $P \subset{ }^{\gamma} Q \equiv \gamma Q \gamma^{-1}$.

For $P \in \mathcal{P}$, let $r(P)$ denote the parabolic rank; this is the number of proper parabolic $\mathbb{Q}$-subgroups in a maximal chain $P=P_{r}<P_{r-1}<\cdots P_{1}<G$. Let $\left|\Delta_{P}\right|^{\circ}$ denote an open simplex of dimension $r(P)-1$. We can now crudely describe the strata and their links. Each stratum $X_{P}$ of $\widehat{X}$ will be a locally symmetric space; in the above example this was $S^{1}$. Each stratum $Y_{P}$ of $\bar{X}$ is a flat bundle over $X_{P}$ with general fiber a compact nilmanifold $\mathcal{N}_{P}^{\prime}$; in the above example the nilmanifold was $T^{2}$. The links are now themselves stratified. 
The corresponding data for a Satake compactification of $X=\Gamma \backslash D$ is in general more complicated; we will give Zucker's realization of $X^{*}$ as a quotient of $\widehat{X}[\mathbf{8 2}]$. The construction depends on the choice of a certain representation $U$ of $G$ which must satisfy a condition called "geometric rationality" $[\mathbf{2 5}]$. This data induces a decomposition $X_{P}=X_{P, \ell} \times X_{P, h}$ (modulo a finite quotient) for each stratum $X_{P}$ of $\widehat{X}$. The strata of $X^{*}$ will be the various $X_{P, h}$, and the quotient map $\widehat{X} \stackrel{\pi}{\rightarrow} X^{*}$ is induced by projection, stratum by stratum. The situation is complicated by the fact that several $P \in \mathcal{P}$ may yield the same stratum in $X^{*}$; we index the boundary stratum $X_{P, h}$ of $X^{*}$ by the maximal such $P$, denoted $P^{\dagger}$. Let $\mathcal{P}^{*}=\left\{P^{\dagger} \mid P \in \mathcal{P}\right\}$.

We can summarize the above discussion in the following table; for simplicity we indicate just the smooth locus of the links.

\begin{tabular}{c|c|c} 
& Boundary stratum associated to $P$ & Link $^{\circ}$ \\
\hline $\bar{X}$ & $Y_{P}=$ flat $\mathcal{N}_{P^{\prime}}^{\prime}$-bundle over $X_{P}$ & $\left|\Delta_{P}\right|^{\circ}$ \\
$\widehat{X}$ & $X_{P}$ & $\mathcal{N}_{P}^{\prime} \times\left|\Delta_{P}\right|^{\circ}$ \\
$X^{*}$ & $X_{P^{\dagger}, h}$ & $\left(\mathcal{N}_{P^{\dagger}}^{\prime} \times\left|\Delta_{P^{\dagger}}\right|^{\circ}\right)$-bundle over $X_{P^{\dagger}, \ell}$
\end{tabular}

\section{The Borel-Serre compactification}

As in $\S 3$, we consider a locally symmetric space $X=\Gamma \backslash D$ where $D=G / K A_{G}$. In this section we outline the actual construction of $\bar{X}$ due to Borel and Serre [16]. The construction uses the language of algebraic groups, in particular their parabolic subgroups, for which good references are the texts of Borel [12] and Humphreys [40], as well as the paper by Borel and Tits [17]. For the reduction theory of arithmetic groups, one may consult Borel's book [9]. We illustrate the discussion with the example of $G=\mathrm{GL}_{n}(\mathbb{R})$ and $\Gamma=\mathrm{GL}_{n}(\mathbb{Z})$. In this case $A_{G}$ is the subgroup of positive scalar matrices and we may pick $K=\mathrm{O}(n)$. The symmetric space $D=\mathrm{GL}_{n}(\mathbb{R}) / \mathrm{O}(n) A_{G}$ is the space of positive definite $n \times n$ symmetric matrices modulo homothety and $X=\Gamma \backslash D$ is the moduli of positive definite quadratic forms on $\mathbb{Z}^{n}$ modulo homothety and isometry.

Let $P$ be a parabolic $\mathbb{Q}$-subgroup, let $N_{P}$ be its unipotent radical and $L_{P}=$ $P / N_{P}$ its Levi quotient. We can when necessary lift $L_{P}$ to a subgroup $\widetilde{L}_{P}$ of $P$ such that $P=\widetilde{L}_{P} N_{P}$. In the case $G=\mathrm{GL}_{n}(\mathbb{R})$, any such $P$ is conjugate under $\mathrm{GL}_{n}(\mathbb{Q})$ to the group of block upper-triangular matrices associated to a partition $n=n_{0}+\cdots+n_{r}, n_{i} \geq 0$. A lift of the Levi quotient is the group of block diagonal matrices, $\mathrm{GL}_{n_{0}}(\mathbb{R}) \times \cdots \times \mathrm{GL}_{n_{r}}(\mathbb{R})$, while the unipotent radical $N_{P}$ consists of those block upper-triangular matrices with identity matrices along the diagonal; see Figure 4. Conjugation by elements of $N_{P}$ would take one lift $\widetilde{L}_{P}$ to all possible such lifts. The stabilizer of a basepoint $x_{0} \in D$ has the form $K A_{G}$ where $K$ is a maximal compact subgroup. Thus the choice of a basepoint determines a maximal compact subgroup $K$ with a corresponding Cartan involution $\theta$; there exists a unique lift $\widetilde{L}_{P}$ of $L_{P}$ which is stable under $\theta$. Such a lift however is not necessarily defined over $\mathbb{Q}$.

Let $A_{P}$ be the identity component of the maximal $\mathbb{Q}$-split torus in the center of $L_{P}$; it has a natural complement $M_{P}={ }^{0} L_{P}$ defined as

$$
{ }^{0} L_{P} \equiv \bigcap_{\alpha \in X\left(L_{P}\right)} \operatorname{ker} \alpha^{2}
$$




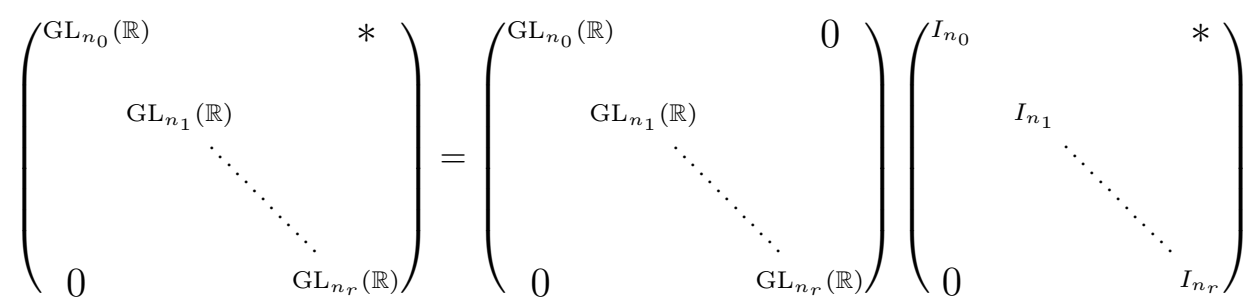

Figure 4. A parabolic subgroup $P=\widetilde{L}_{P} N_{P}$ of $\mathrm{GL}_{n}(\mathbb{R})$

where $X\left(L_{P}\right)$ denotes the (rational) characters of $L_{P}$ defined over $\mathbb{Q}$. Thus $L_{P}=$ $M_{P} A_{P}$ and this decomposition lifts to $\widetilde{L}_{P}=\widetilde{M}_{P} \widetilde{A}_{P}$. In the example, $\widetilde{A}_{P}$ consists of diagonal matrices which are positive scalar matrices in each block and $\widetilde{M}_{P}$ consists of block diagonal matrices $\mathrm{SL}_{n_{0}}^{ \pm}(\mathbb{R}) \times \cdots \times \mathrm{SL}_{n_{r}}^{ \pm}(\mathbb{R})$, where $\mathrm{SL}_{n_{i}}^{ \pm}(\mathbb{R})$ denotes matrices with determinant \pm 1 .

There is a natural inclusion $A_{G} \subseteq A_{P}$ and a complement $A_{P}^{G} \subseteq A_{P}$ given as the joint kernel of the characters in $X(G)$. In our standard example, $a=$ $\operatorname{diag}\left(\lambda_{0} \cdot I_{n_{0}}, \ldots, \lambda_{r} \cdot I_{n_{r}}\right) \in A_{P}$ lies in $A_{G}$ if and only if $\lambda_{0}=\cdots=\lambda_{r}$ and $a$ lies in $A_{P}^{G}$ if and only if $\operatorname{det} a=1$. Since $A_{P}^{G}$ is $\mathbb{Q}$-split, it has coordinates given by characters of $P$ defined over $\mathbb{Q}$. In the next paragraph we will define an important and canonical set of characters $\Delta_{P}$ so that we have an isomorphism

$$
A_{P}^{G} \stackrel{\longrightarrow}{\longrightarrow}\left(\mathbb{R}_{+}^{*}\right)^{\Delta_{P}}, \quad a \mapsto\left(a^{\alpha_{1}}, \ldots, a^{\alpha_{r}}\right) .
$$

There is an adjoint action of $z \in L_{P}$ on the Lie algebra $\mathfrak{n}_{P}$ of $N_{P}$ given by lifting $z$ to $\widetilde{L}_{P}$ and then acting by the adjoint action. Although this action depends on the lift, its $A_{P}^{G}$-weights (which are induced from characters of $P$ ) do not. For $P$ a minimal parabolic $\mathbb{Q}$-subgroup these are the positive roots of a root system, namely the $\mathbb{Q}$-root system of $G$, but this does not hold true in general. However it is still true that if we let $\Delta_{P}=\left\{\alpha_{1}, \ldots, \alpha_{r}\right\}$ be the indecomposable elements among the $A_{P}^{G}$-weights of $\mathfrak{n}_{P}$, then all $A_{P}^{G}$-weights of $\mathfrak{n}_{P}$ can be expressed uniquely as non-negative integral linear combinations of elements of $\Delta_{P}$. We call the weights in $\Delta_{P}$ the simple roots even though they may not generate a root system. There is also a "dual" basis $\widehat{\Delta}_{P}=\left\{\beta_{1}, \ldots, \beta_{r}\right\}$ which in the case of a minimal parabolic $\mathbb{Q}$-subgroup is simply the set of fundamental weights. In our example, we have

$$
a^{\alpha_{i}}=\lambda_{i} / \lambda_{i-1} \quad \text { and } \quad a^{\beta_{i}}=1 /\left(\lambda_{0} \cdots \lambda_{i-1}\right)
$$

for $a=\operatorname{diag}\left(\lambda_{0} \cdot I_{n_{0}}, \ldots, \lambda_{r} \cdot I_{n_{r}}\right) \in A_{P}^{G}$.

For later use we single out two subsets of $A_{P}^{G}$, the strictly dominant cone

$$
A_{P}^{G+} \equiv\left\{a \in A_{P}^{G} \mid a^{\alpha}>1 \text { for all } \alpha \in \Delta_{P}\right\}
$$

and the negative codominant cone,

$$
{ }^{-} A_{P}^{G} \equiv\left\{a \in A_{P}^{G} \mid a^{\beta} \leq 1 \text { for all } \beta \in \widehat{\Delta}_{P}\right\} .
$$

The Lie algebra $\mathfrak{a}_{P}^{G}$ of $A_{P}^{G}$, endowed with a Weyl group invariant inner product, is illustrated in Figure 5(a) in the case of a minimal parabolic $\mathbb{Q}$-subgroup of $\mathrm{GL}_{3}(\mathbb{R})$ together with the subsets $\mathfrak{a}_{P}^{G+} \equiv \log A_{P}^{G+}$ and ${ }^{-} \mathfrak{a}_{P}^{G} \equiv \log { }^{-} A_{P}^{G}$. The figure also includes $\Delta_{P}$ and $\widehat{\Delta}_{P}$, identified with elements of $\mathfrak{a}_{P}^{G *}$ and then transfered to $\mathfrak{a}_{P}^{G}$ via the inner product. 


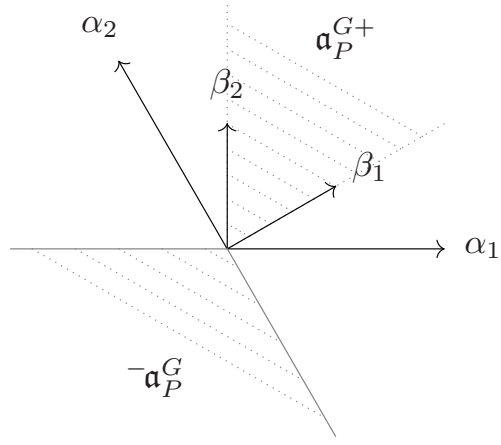

(a)

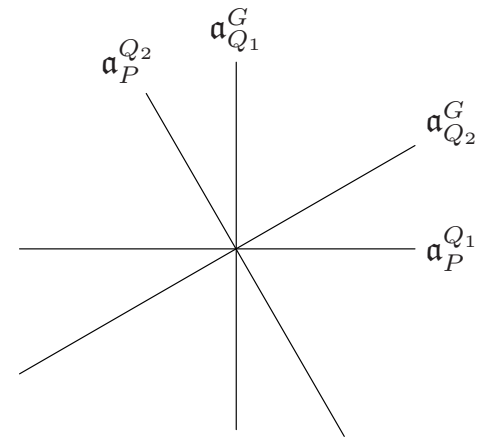

(b)

Figure 5. $\mathfrak{a}_{P}^{G}$, its subcones, and its subspaces for $P$ a minimal parabolic subgroup of $\mathrm{GL}_{3}(\mathbb{R})$. (Here $\Delta_{P}=\left\{\alpha_{1}, \alpha_{2}\right\}$ and $\Delta_{P}^{Q_{i}}=$ $\left\{\alpha_{i}\right\}$.)

The parabolic $\mathbb{Q}$-subgroups $Q$ containing $P$ are in one-to-one order preserving correspondence with subsets $\Delta_{P}^{Q}$ of $\Delta_{P}$. Namely, consider $Q=\widetilde{L}_{Q} N_{Q} \supseteq P=$ $\widetilde{L}_{P} N_{P}$; we can decompose $N_{P}=\left(N_{P} \cap \widetilde{L}_{Q}\right) N_{Q}$. Define $\Delta_{P}^{Q}$ to consist of those $\alpha \in \Delta_{P}$ whose weight space lies in $\mathfrak{n}_{P} \cap \widetilde{\mathfrak{l}}_{Q}$ as opposed to $\mathfrak{n}_{Q}$. For such a $Q$, there is a natural identification

$$
A_{Q}^{G} \equiv \bigcap_{\alpha \in \Delta_{P}^{Q}} \operatorname{ker} \alpha \subseteq A_{P}^{G}
$$

The set of simple roots $\Delta_{Q}$ for $Q$ is the set of restrictions $\left.\alpha\right|_{A_{Q}^{G}}$ for $\alpha \in \Delta_{P} \backslash \Delta_{P}^{Q}$. Furthermore there is a complementary subgroup

$$
A_{P}^{Q} \equiv \bigcap_{\alpha \in \Delta_{P} \backslash \Delta_{P}^{Q}} \operatorname{ker} \beta_{\alpha} \subseteq A_{P}^{G}
$$

to $A_{Q}^{G}$; here $\beta_{\alpha} \in \widehat{\Delta}_{P}$ is the dual element to $\alpha \in \Delta_{P}$. The group $A_{P}^{Q}$ may also be viewed as the analogue of $A_{P}^{G}$ for the parabolic $\mathbb{Q}$-subgroup $P / N_{Q}$ of $L_{Q}$. We have a direct decomposition

$$
A_{P}^{G}=A_{Q}^{G} \times A_{P}^{Q} .
$$

The corresponding decomposition of the Lie algebra in the example of $\mathrm{GL}_{3}(\mathbb{R})$ is illustrated in Figure 5(b). Note that the closure of the strictly dominant cone $A_{P}^{G+}$ is stratified with strata $\left\{A_{Q}^{G+}\right\}_{Q \supseteq P}$, while $\left\{{ }^{-} A_{P}^{Q}\right\}_{Q \supseteq P}$ are the closures of the strata of the negative codominant cone ${ }^{-} A_{P}^{G}$.

The compactification $\bar{X}$ will be the quotient by $\Gamma$ of a bordification $\bar{D}$ which will be constructed in three steps.

6.1. The first step is to form a partial bordification $\bar{A}_{P}^{G}$ of $A_{P}^{G}$, a "corner", by allowing the coordinates in (11) to attain infinity:

$$
\bar{A}_{P}^{G} \underset{\sim}{\longrightarrow}\left(\mathbb{R}_{+}^{*} \cup\{\infty\}\right)^{\Delta_{P}}, \quad a \mapsto\left(a^{\alpha_{1}}, \ldots, a^{\alpha_{r}}\right) .
$$




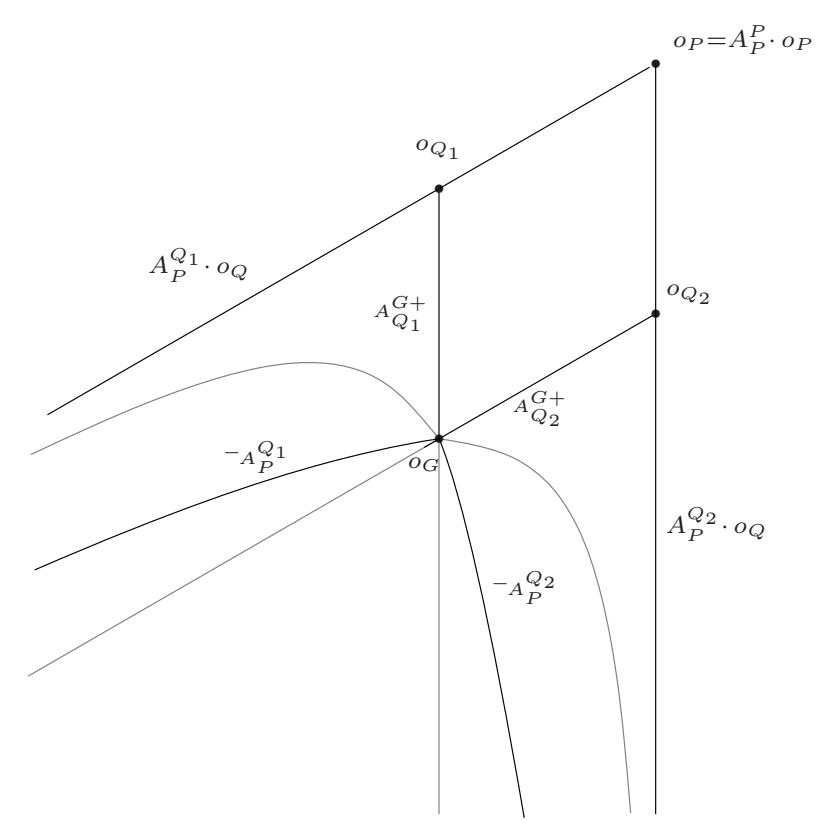

Figure $6 . \bar{A}_{P}^{G}$ for $P$ a minimal parabolic subgroup of $\mathrm{GL}_{3}(\mathbb{R})$

The corner $\bar{A}_{P}^{G}$ is given a real-analytic structure by means of the inverses of these coordinates: $\bar{A}_{P}^{G} \rightarrow\left(\mathbb{R}_{\geq 0}\right)^{\Delta_{P}}, a \mapsto\left(a^{-\alpha_{1}}, \ldots, a^{-\alpha_{r}}\right)$. For $Q \supseteq P$, the inclusion $A_{Q}^{G} \hookrightarrow A_{P}^{G}$ extends to the partial bordifications, $\bar{A}_{Q}^{G} \hookrightarrow \bar{A}_{P}^{G}$. The action of $A_{P}^{G}$ on itself by multiplication extends naturally to an action of $A_{P}^{G}$ on $\bar{A}_{P}^{G}$. In fact $\bar{A}_{P}^{G}$ is a semigroup. Set $\bar{A}_{P}^{G+}$ to be the subset where all coordinates are greater than 1 .

Let $o_{Q} \in \bar{A}_{P}^{G}$ be the point with coordinates $\{\infty\}^{\Delta_{P} \backslash \Delta_{P}^{Q}} \times\{1\}^{\Delta_{P}^{Q}}$; this point has stabilizer $A_{Q}^{G}$ so the orbit it generates is $A_{P}^{Q} \cdot o_{Q}$. The space $\bar{A}_{P}^{G}$ is stratified by these $A_{P}^{G}$-orbits:

$$
\bar{A}_{P}^{G}=\coprod_{Q \supseteq P} A_{P}^{Q} \cdot o_{Q}
$$

Topologically $\bar{A}_{P}^{G}$ is homeomorphic to a cone $c\left(\left|\Delta_{P}\right|\right)$ on the simplex $\left|\Delta_{P}\right|$. (We denote by $\left|\Delta_{P}\right|$ the $(r-1)$-dimensional closed simplex whose vertices are identified with $\alpha \in \Delta_{P}$.) The vertex of the cone is $o_{P}=\{\infty\}^{\Delta_{P}}$ and the sub-cone $c\left(\left|\Delta_{P}^{Q}\right|\right)$ corresponds to the closure of the orbit $A_{P}^{Q} \cdot o_{Q}$.

All of the above illustrated in Figure 6 for $P$ a minimal parabolic subgroup in $G=\mathrm{GL}_{3}(\mathbb{R})$. The figure is drawn using analytic coordinates based at $o_{P}$ and chosen so that at $o_{G}$ the diagram accurately reflects the geometry of a Weyl-group invariant inner product on the tangent space $\mathfrak{a}_{P}^{G}$. It is important to note that whereas the two 1-parameter subgroups $A_{Q_{1}}^{G}$ and $A_{Q_{2}}^{G}$ each intersect the boundary at infinity in 1 point, neither of the subgroups $A_{P}^{Q_{1}}$ or $A_{P}^{Q_{2}}$ intersect the boundary at all. This is due to the fact that the two simple roots are not orthogonal.

6.2. The second step in the construction of $\bar{D}$ is to extend the partial bordification of $A_{P}^{G}$ to one of $D$. To do this, note that even though $A_{P}$ is not naturally 
a subgroup of $G$, Borel and Serre define an action of $A_{P}$ on $D$ called the geodesic action $[\mathbf{1 6}, \S 3]$. To describe it, fix a basepoint in $D$ which determines a maximal compact subgroup $K \subseteq G$, a Cartan involution $\theta$, and a $\theta$-stable lift $\widetilde{L}_{P}$ of $L_{P}$. Note that $G=P K$, that is, $P$ acts transitively on $D$; this is a generalization of Gram-Schmidt orthonormalization. Every point $x \in D$ can thus be written as $x=p K A_{G}$ for some $p \in P$, and the geodesic action of $a \in A_{P}$ on $x$ is given by

$$
a \mathbf{o} x \equiv p \tilde{a} K A_{G},
$$

where $\tilde{a} \in \tilde{A}_{P}$ is the lift of $a$. The geodesic action is independent of the choice of basepoint and commutes with the usual action of $P$.

Let $e_{P} \equiv A_{P}^{G} \backslash D$ be obtained by collapsing the orbits of the geodesic action of $A_{P}^{G}$ to points. We will further discuss the structure of $e_{P}$ below. The quotient map $r_{P}: D \rightarrow e_{P}$ is a $A_{P}^{G}$-principal bundle; natural trivializing sections are given by the orbits of ${ }^{0} P \equiv \bigcap_{\alpha \in X(P)}$ ker $\alpha^{2}$. Thus if we let $\mathcal{A}_{P}^{G} \equiv{ }^{0} P \backslash D$ be the $A_{P}^{G}$-homogeneous space obtained by collapsing these sections to points, there is a natural product decomposition

$$
D=\mathcal{A}_{P}^{G} \times e_{P} .
$$

This generalizes the product decomposition of the upper half-plane into geodesics tending to $\infty$ (rays parallel to the positive $y$-axis) times horocycles at $\infty$ (lines parallel to the $x$-axis). For $P$ a minimal parabolic $\mathbb{R}$-subgroup this is the decomposition induced from the Iwasawa decomposition of $G$.

The space $\mathcal{A}_{P}^{G}$ is an affine version of $A_{P}^{G}$; given a choice of basepoint in $D$, there is a canonical isomorphism of $A_{P}^{G}$-homogeneous spaces $\mathcal{A}_{P}^{G} \cong A_{P}^{G}$ such that the class of the basepoint maps to the identity. We can then define a bordification $\overline{\mathcal{A}}_{P}^{G}$ such that this isomorphism extends to an isomorphism $\overline{\mathcal{A}}_{P}^{G} \cong \bar{A}_{P}^{G}$. The resulting $A_{P}^{G}$-space (in fact $\bar{A}_{P}^{G}$-space) is independent of the choice of basepoint. Note that although the points $o_{Q} \in \bar{A}_{P}^{G}$ do not uniquely determine points in $\overline{\mathcal{A}}_{P}^{G}$ (except for $Q=P$ ), the orbits $A_{P}^{Q} \cdot o_{Q} \subseteq \bar{A}_{P}^{G}$ do indeed define well-defined orbits in $\overline{\mathcal{A}}_{P}^{G}$. We will again use the notation $A_{P}^{Q} \cdot o_{Q}$ to denote the corresponding orbit of $\overline{\mathcal{A}}_{P}^{G}$.

Borel and Serre construct a partial bordification $D(P)$ by replacing $\mathcal{A}_{P}^{G}$ in the above decomposition with $\overline{\mathcal{A}}_{P}^{G}$ :

$$
D(P)=\overline{\mathcal{A}}_{P}^{G} \times e_{P} .
$$

We consider $e_{P}$ as a stratum of $D(P)$ by identifying it with $o_{P} \times e_{P}=\{\infty\}^{\Delta_{P}} \times e_{P}$; the link of any point in $e_{P}$ is $\left|\Delta_{P}\right|$. The projection map

$$
D(P)=\overline{\mathcal{A}}_{P}^{G} \times e_{P} \longrightarrow\{\infty\}^{\Delta_{P}} \times e_{P} \equiv e_{P}
$$

is called geodesic retraction.

6.3. The third and final step in the construction of $\bar{D}$ is to note that the construction above of the partial bordification is functorial in the sense that for $P \subseteq Q$ we can canonically identify $D(Q)$ with an open subset of $D(P)$. Indeed $e_{Q} \subseteq D(Q)$ corresponds to $\left(A_{P}^{Q} \cdot o_{Q}\right) \times e_{P} \subseteq D(P)$ and so $D(P)$ may be re-expressed as $\coprod_{Q \supseteq P} e_{Q}$. Borel and Serre then define a full bordification of $D$ by setting

$$
\bar{D}=\bigcup_{P} D(P)=\coprod_{P} e_{P}
$$

where the union is over all parabolic $\mathbb{Q}$-subgroups $P$ and the above identifications are taken into account. Let $\bar{e}_{P}$ denote the closure of $e_{P}$ in $\bar{D}$. 
The action of $G$ on $D$ extends to an action of $G(\mathbb{Q})$ on $\bar{D}$ and the Borel-Serre compactification is defined by

$$
\bar{X} \equiv \Gamma \backslash \bar{D} .
$$

Given a parabolic $\mathbb{Q}$-subgroup $P$, the various strata $e_{\gamma P}$ of $\bar{D}$ for $\gamma \in \Gamma$ become identified to one stratum $Y_{P}$ in $\bar{X}$. Furthermore reduction theory shows that the identifications induced by $\Gamma$ on $e_{P}$, and indeed even on a small neighborhood of $\bar{e}_{P}$, are already induced by $\Gamma_{P} \equiv \Gamma \cap P$. Thus there is a stratification

$$
\bar{X}=\coprod_{P \in \mathcal{P}} Y_{P}, \quad \text { where } Y_{P} \equiv \Gamma_{P} \backslash e_{P} .
$$

The geodesic retraction (13) extends to a projection, also called geodesic retraction,

$$
V \longrightarrow Y_{P}
$$

where $V$ is a sufficiently small neighborhood of $Y_{P}$ in $\bar{X}$. In fact, if $O_{P}$ is a relatively compact open subset of $Y_{P}$ and $\widetilde{O}_{P}$ is its inverse image in $e_{P}$, reduction theory shows that there exists $s_{P} \in \mathcal{A}_{P}^{G}$ such that the identifications induced by $\Gamma$ on

$$
\widetilde{W}_{P} \equiv\left(\bar{A}_{P}^{G+} \cdot s_{P}\right) \times \widetilde{O}_{P} \subseteq D(P)
$$

agree with those induced by $\Gamma_{P}$. However $\Gamma_{P}$ acts only on the second factor. Thus

$$
W_{P} \equiv \Gamma_{P} \backslash \widetilde{W}_{P}=\left(\bar{A}_{P}^{G+} \cdot s_{P}\right) \times O_{P} \subseteq \Gamma_{P} \backslash D(P)
$$

may be identified with a subset of $\bar{X}$; the geodesic retraction is projection on the second factor. The open subset $W_{P}$ is called a cylindrical set; for appropriate choices of $O_{P}$ and $s_{P}$ (for all $P \in \mathcal{P}$ ) one obtains a cylindrical cover

$$
\bar{X}=\bigcup_{P \in \mathcal{P}} W_{P} .
$$

Let $x \in Y_{P}$. If $O_{P}$ is a ball neighborhood $B$ of $x$ in $Y_{P}$ the construction above yields a neighborhood in $\bar{X}$ of $x$ which is homeomorphic (as a stratified space) to $B \times c\left(\left|\Delta_{P}\right|\right)$. (Here we have interchanged the factors in (15).) As $B$ shrinks and $s_{P}$ tends to $o_{P}$ we obtain a basis of local neighborhoods of $x$. The link $\left|\Delta_{P}\right|$ is itself stratified by its intersections with the strata $Y_{Q}>Y_{P}$; this stratification is

$$
\left|\Delta_{P}\right|=\coprod_{Q>P}\left|\Delta_{P}^{Q}\right|^{\circ}
$$

where $\left|\Delta_{P}^{Q}\right|^{\circ}$ denotes the open face with vertices $\Delta_{P}^{Q}$.

\section{The reductive Borel-Serre compactification}

We outline the construction of $\widehat{X}$ which was introduced by Zucker [81]; see also $[30]$.

Recall that $e_{P}$ is a homogeneous space for ${ }^{0} P$. Let $D_{P}=N_{P} \backslash e_{P}$ be obtained by collapsing the orbits of the unipotent radical; it a symmetric space for $L_{P}$, namely $D_{P}=L_{P} / K_{P} A_{P}$ where $K_{P}=K \cap P$. The quotient map $e_{P} \rightarrow D_{P}$ is a principal $N_{P}$-bundle. We can define a geodesic action of $L_{P}$ on $D[6 \mathbf{6 1}, \S 1.1]^{1}$ which extends that of $A_{P}$; the orbits of the induced geodesic action of ${ }^{0} L_{P}$ on $e_{P}$ yield natural

\footnotetext{
${ }^{1}$ Precise references to numbered results in [61] refer to version 2 as posted on the arXiv. The numbering in [61] may change in the published version due to revision or a possible splitting of the paper.
} 
trivializing sections of $e_{P} \rightarrow D_{P}$. We let $\mathcal{N}_{P}$ be obtained from $e_{P}$ be collapsing these sections; thus there is a natural decomposition

$$
e_{P}=D_{P} \times \mathcal{N}_{P}
$$

More concretely, the decomposition ${ }^{0} P=\widetilde{M}_{P} \ltimes N_{P}$ (which depends on the choice of basepoint $x_{0} \in D$ ) induces a decomposition

$$
e_{P} \stackrel{\sim}{\longrightarrow} D_{P} \times N_{P}, \quad n m K_{P} \mapsto\left(m K_{P}, n\right), \quad\left(n \in N_{P}, m \in \widetilde{M}_{P}\right) .
$$

This agrees with (17) under the canonical isomorphism of $N_{P}$-homogeneous spaces $\mathcal{N}_{P} \cong N_{P}$ which sends the class of $x_{0}$ to the identity. In the example considered in $\S 6$ (see Figure 4 ), $D_{P}$ is the product of the spaces of positive definite $n_{i} \times n_{i}$ symmetric matrices modulo homothety, for $i=0, \ldots, r$.

The action of $\gamma \in \Gamma_{N_{P}} \equiv \Gamma \cap N_{P} \subseteq \Gamma_{P}$ is solely on the second factor of (17); thus we obtain

$$
\Gamma_{N_{P}} \backslash e_{P}=D_{P} \times \mathcal{N}_{P}^{\prime}, \quad \text { where } \mathcal{N}_{P}^{\prime} \equiv \Gamma_{N_{P}} \backslash \mathcal{N}_{P} .
$$

To achieve the full quotient $Y_{P}=\Gamma_{P} \backslash e_{P}$ we need to now take the quotient by $\Gamma_{L_{P}} \equiv \Gamma_{P} / \Gamma_{N_{P}}$. An element $\gamma \in \Gamma_{L_{P}}$ acts in the usual way on the first factor $D_{P}$ but in view of (18) it also conjugates the second factor. Thus the trivial $N_{P^{-}}$ principal bundle $e_{P} \rightarrow D_{P}$ descends to a flat bundle of the arithmetic quotients, called the nilmanifold fibration:

$$
\underset{X_{P} \equiv \Gamma_{L_{P} \backslash D_{P}}^{Y_{P}}}{\downarrow_{\text {flat } \mathcal{N}_{P}^{\prime} \text {-bundle }}}
$$

The reductive Borel-Serre compactification $\widehat{X}$ is obtained by collapsing the fibers of the nilmanifold fibration on each stratum $Y_{P}$ of $\bar{X}$. Thus there is a quotient map $\bar{X} \rightarrow \widehat{X}$ which is stratified with respect to the stratification

$$
\widehat{X}=\coprod_{P \in \mathcal{P}} X_{P}
$$

The closure of a stratum is $\widehat{X}_{P}$, the reductive Borel-Serre compactification of $X_{P}$.

In order to understand the structure of $\widehat{X}$ near a point $x \in X_{P}$, we first look at the inverse image of $x$ in $\bar{X}$. This is a full nilmanifold fiber $\mathcal{N}_{P}^{\prime}$ in $Y_{P}$ which has a basis of neighborhoods of the form

$$
\widetilde{U}=B \times \mathcal{N}_{P}^{\prime} \times c\left(\left|\Delta_{P}\right|\right),
$$

where $B$ maps diffeomorphically to a ball neighborhood of $x$ in $X_{P}$. (Again we have interchanged the factors in (15).) The strata of $\widetilde{U}$ are

$$
\widetilde{U} \cap Y_{Q}= \begin{cases}B \times \mathcal{N}_{P}^{\prime} \times c\left(\left|\Delta_{P}\right|\right)^{\circ} & Q=G, \\ B \times \mathcal{N}_{P}^{\prime} \times c\left(\left|\Delta_{P}^{Q}\right|\right)^{\circ} & P<Q<G, \\ B \times \mathcal{N}_{P}^{\prime} \times c(\emptyset) & Q=P,\end{cases}
$$

where $c(\emptyset)$ denotes the vertex of the cone and $c\left(\left|\Delta_{P}^{Q}\right|\right)^{\circ} \equiv c\left(\left|\Delta_{P}^{Q}\right|^{\circ}\right) \backslash c(\emptyset)$. 
A neighborhood $U$ of $x$ is obtained by projecting $\widetilde{U}$ to $\widehat{X}$. Since the $\mathcal{N}_{Q}^{\prime}$-fibers in $Y_{Q}$ need to be collapsed for each $Q \geq P$, (19) is replaced by

$$
U \cap X_{Q}= \begin{cases}B \times \mathcal{N}_{P}^{\prime} \times c\left(\left|\Delta_{P}\right|\right)^{\circ} & Q=G, \\ B \times \mathcal{N}_{P}^{\prime Q} \times c\left(\left|\Delta_{P}^{Q}\right|\right)^{\circ} & P<Q<G, \\ B \times c(\emptyset) & Q=P,\end{cases}
$$

where $\mathcal{N}_{P}^{\prime Q} \equiv\left(\Gamma_{N_{P}} / \Gamma_{N_{Q}}\right) \backslash\left(N_{Q} \backslash \mathcal{N}_{P}\right)$. In particular, over $X=X_{G}$ no collapsing occurs, while over $X_{P}$ the entire $\mathcal{N}_{P}^{\prime}$ factor is collapsed to a point.

The link of the stratum $X_{P} \subseteq \widehat{X}$ is thus

$$
\left(\mathcal{N}_{P}^{\prime} \times\left|\Delta_{P}\right|\right) / \sim=\coprod_{Q>P} \mathcal{N}_{P}^{\prime Q} \times\left|\Delta_{P}^{Q}\right|^{\circ},
$$

where $\left(\Gamma_{N_{P}} n, a\right) \sim\left(\Gamma_{N_{P}} n^{\prime}, a\right)$ if $a \in\left|\Delta_{P}^{Q}\right|^{\circ}$ and $n^{\prime}=u n$ for some $u \in N_{Q}$.

\section{Satake compactifications}

The Satake compactification $X^{*}$ depends on an irreducible representation $(\sigma, U)$ of $G$. The construction proceeds in several stages. Satake [67] first constructs a compactification $_{\mathbb{R}} D^{*}$ of $D$ depending on $(\sigma, U)$. The compactification ${ }_{\mathbb{R}} D^{*}$ is a disjoint union of subsets called real boundary components. The action of $G$ on $D$ extends to an action on ${ }_{\mathbb{R}} D^{*}$ which permutes the boundary components. In $[\mathbf{6 8}]$, Satake defines $D^{*} \subseteq{ }_{\mathbb{R}} D^{*}$ to be the union of certain of these boundary components, called rational boundary components, and shows that $G(\mathbb{Q})$ acts on $D^{*}$. He defines the Satake topology on $D^{*}$ and sets $X^{*} \equiv \Gamma \backslash D^{*}$. These steps, and certain assumptions that have to be made on $(\sigma, U)$, are described in more detail below. In addition to the above papers of Satake, references are the papers of Borel [8], Casselman [25], and Zucker [82].

\subsection{Assume}

(i) $(\sigma, U)$ is nontrivial on each $\mathbb{R}$-simple component;

(ii) the maximal $\mathbb{Q}$-split torus and the maximal $\mathbb{R}$-split torus in the center of $G$ coincide.

(This second assumption is not essential but it simplifies the exposition.) Give $U$ an admissible inner product, that is, one for which

$$
\sigma(g)^{*}=\sigma(\theta g)^{-1}, \quad \text { for all } g \in G ;
$$

here $\theta$ is the Cartan involution associated to a choice of maximal compact subgroup $K \subseteq G$. There is an action of $G$ on the real vector space $\operatorname{Herm}(U)$ of Hermitian endomorphisms of $U$ given by

$$
S \mapsto \sigma(g) S \sigma(g)^{*}, \quad \text { for } S \in \operatorname{Herm}(U), g \in G .
$$

In particular $g \in K$ acts by conjugation by $\sigma(g)$, while $g \in A_{G}$ acts by a real scalar (since the center of $G$ acts by a rational character on an irreducible representation). Thus if we consider the induced projective representation, the class of the identity $[I] \in \mathbb{P} \operatorname{Herm}(U)$ is fixed by $K A_{G}$ and there is a well-defined map

$$
D=G / K A_{G} \longrightarrow \mathbb{P} \operatorname{Herm}(U), \quad x=g K A_{G} \mapsto g \cdot[I]=\sigma(g) \sigma(g)^{*} .
$$

This map is an embedding by the assumptions above and ${ }_{\mathbb{R}} D^{*}$ is defined to be the closure of the image of $D$ in $\mathbb{P} \operatorname{Herm}(U)$. 
An important class of examples are the Hermitian symmetric spaces, in which $D$ has a $G$-invariant complex structure. In this case the $\mathbb{R}$-root system is always type $C_{r}$ or $B C_{r}$ and there is a unique simple $\mathbb{R}$-root $\alpha_{r}$ at the end of the diagram with a double bond (if the type is $C_{2}$ (resp. $B C_{2}$ ), $\alpha_{2}$ is the long (resp. short) simple root). If the highest restricted weight of $(\sigma, U)$ is a multiple of the fundamental weight dual to $\alpha_{r}$, the compactification ${ }_{\mathbb{R}} D^{*}$ is homeomorphic to the closure of $D$ in $\mathbb{C}^{N}$ under Harish-Chandra's embedding as a bounded symmetric domain [38]. This is called the natural compactification.

8.2. A real boundary component is by definition the set of points of ${ }_{\mathbb{R}} D^{*}$ fixed by the unipotent radical of a parabolic $\mathbb{R}$-subgroup of $G$; if $P$ is a parabolic $\mathbb{R}$-subgroup, we let $D_{P, h} \equiv \operatorname{Fix}\left(N_{P}\right) \cap_{\mathbb{R}} D^{*}$ denote the associated real boundary component. The compactification ${ }_{\mathbb{R}} D^{*}$ is the disjoint union of its real boundary components. The action of $P$ on $D_{P, h}$ descends to an action of the Levi quotient $L_{P}$ and there is an almost direct product decomposition (direct product of subgroups modulo finite subgroup) of reductive $\mathbb{R}$-subgroups

$$
L_{P}=\widetilde{L}_{P, h} \times L_{P, \ell},
$$

where $L_{P, \ell}$ is the subgroup fixing $D_{P, h}$ pointwise and the induced action of $L_{P, h} \equiv$ $L_{P} / L_{P, \ell}$ on $D_{P, h}$ is that of a real reductive group on its associated symmetric space. This decomposition yields a product decomposition of the associated symmetric spaces,

$$
D_{P}=D_{P, h} \times D_{P, \ell} .
$$

In the case of the natural compactification of a bounded symmetric domain in $\mathbb{C}^{N}$, the real boundary components, which are again Hermitian symmetric spaces, can also be described as the holomorphic arc components: two points are in the same holomorphic arc component if there are a finite number of holomorphic maps of $\{z \in \mathbb{C}|| z \mid<1\}$ into the closure of $D$ for which the union of the images is connected and contains the two points.

8.3. We can be more specific about the subgroups $L_{P, \ell}$ and $\widetilde{L}_{P, h}$, in particular, their root systems. First some notation. Choose a maximal $\mathbb{R}$-split torus ${ }_{\mathbb{R}} A$ of $L_{P}$; via an appropriate lift we may identify it with a maximal $\mathbb{R}$-split torus of $G$. Let ${ }_{\mathbb{R}} \Delta$ be the simple $\mathbb{R}$-roots of $G$ with respect to a fixed positive system of $\mathbb{R}$-roots which contains the roots in $\mathfrak{n}_{P}$. To any set of $\mathbb{R}$-weights $\psi$ is associated a graph with vertex set $\psi$ and an edge between $\alpha, \beta \in \psi$ if $(\alpha, \beta) \neq 0$. (Here we view weights as belonging to $\mathfrak{R}_{\mathbb{R}} \mathfrak{a}^{*}$ endowed with a Weyl group invariant inner product.) The set $\psi$ is connected if the associated graph is connected.

Let ${ }_{\mathbb{R}} \mu$ be the highest weight of $U$ restricted to ${ }_{\mathbb{R}} A$. The simple $\mathbb{R}$-roots ${ }_{\mathbb{R}} \Delta^{P} \subseteq$ ${ }_{\mathbb{R}} \Delta$ of $L_{P}$ may be orthogonally decomposed

$$
{ }_{\mathbb{R}} \Delta^{P}=\kappa\left({ }_{\mathbb{R}} \Delta^{P}\right) \sqcup \zeta\left({ }_{\mathbb{R}} \Delta^{P}\right),
$$

where $\kappa\left({ }_{\mathbb{R}} \Delta^{P}\right)$ is the largest subset of ${ }_{\mathbb{R}} \Delta^{P}$ such that $\kappa\left(\mathbb{R}_{\mathbb{R}} \Delta^{P}\right) \cup\left\{\mathbb{R}_{\mathbb{R}} \mu\right\}$ is connected and $\zeta\left({ }_{\mathbb{R}} \Delta^{P}\right)$ is the complement of $\kappa\left({ }_{\mathbb{R}} \Delta^{P}\right)$ in ${ }_{\mathbb{R}} \Delta^{P}$. Alternatively, a root $\alpha \in_{\mathbb{R}} \Delta^{P}$ is in $\kappa\left({ }_{\mathbb{R}} \Delta^{P}\right)$ (resp. $\left.\zeta\left({ }_{\mathbb{R}} \Delta^{P}\right)\right)$ if it can (resp. cannot) be connected to ${ }_{\mathbb{R}} \mu$ via a chain of weights $\chi_{0} \equiv \alpha, \chi_{1}, \ldots, \chi_{k-1}, \chi_{k} \equiv{ }_{\mathbb{R}} \mu$ satisfying $\chi_{i} \in{ }_{\mathbb{R}} \Delta^{P}$ for $i<k$ and $\left(\chi_{i-1}, \chi_{i}\right) \neq 0$ for $i \leq k$. 


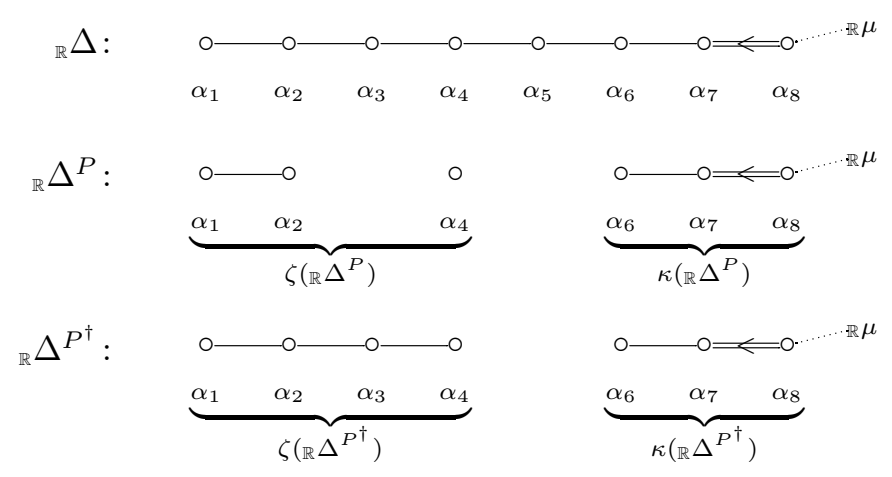

Figure 7. An example of the Dynkin diagrams of the $\mathbb{R}$-root systems of $G, L_{P}$, and $L_{P^{\dagger}}$, where $G=\operatorname{Sp}_{18}(\mathbb{R})$ and $(\sigma, U)$ has highest weight $\mu=\beta_{8}$.

We can now describe the identity component of the centralizer $L_{P, \ell}$ of $D_{P, h}$ as the maximal normal connected $\mathbb{R}$-subgroup of $L_{P}$ with simple $\mathbb{R}$-roots $\zeta\left({ }_{\mathbb{R}} \Delta^{P}\right)$. Similarly the identity component of $\widetilde{L}_{P, h}$ is the minimal normal connected $\mathbb{R}$-subgroup of $L_{P}$ with simple $\mathbb{R}$-roots $\kappa\left({ }_{\mathbb{R}} \Delta^{P}\right)$.

Given a real boundary component $D_{P, h}$, the parabolic subgroup $P$ is not uniquely determined: any parabolic $\mathbb{R}$-subgroup $P^{\prime} \supseteq P$ such that $\kappa\left({ }_{\mathbb{R}} \Delta^{P^{\prime}}\right)=$ $\kappa\left({ }_{\mathbb{R}} \Delta^{P}\right)$ yields the same boundary component. The largest such parabolic $\mathbb{R}$ subgroup is denoted $P^{\dagger}$ and we set

$$
\omega\left({ }_{\mathbb{R}} \Delta^{P}\right) \equiv{ }_{\mathbb{R}} \Delta^{P^{\dagger}} .
$$

The parabolic $\mathbb{R}$-subgroups $R$ for which ${ }_{\mathbb{R}} \Delta^{R}=\omega(\psi)$ for some $\psi \subseteq{ }_{\mathbb{R}} \Delta$ are called saturated; they are the normalizers of the real boundary components. An example for $G=\mathrm{Sp}_{18}(\mathbb{R})$ is pictured in Figure 7; here $D$ is the Siegel generalized upper half-plane (a Hermitian symmetric space) and ${ }_{\mathbb{R}} D^{*}$ is the natural compactification.

8.4. Let $A \subseteq{ }_{\mathbb{R}} A$ be the identity components of a maximal $\mathbb{Q}$-split torus and a maximal $\mathbb{R}$-split torus respectively. The restriction of characters on ${ }_{\mathbb{R}} A$ to characters on $A$ yields a map on simple roots

$$
\rho_{\mathbb{R} / \mathbb{Q}}:{ }_{\mathbb{R}} \Delta \rightarrow \Delta \cup\{0\}
$$

and we set

$$
\epsilon_{\mathbb{R} / \mathbb{Q}}(\Upsilon) \equiv \rho_{\mathbb{R} / \mathbb{Q}}^{-1}(\Upsilon \cup\{0\}), \quad \text { for any } \Upsilon \subseteq \Delta .
$$

We wish now to define a $\Gamma$-invariant union of real boundary components $D^{*} \subseteq$ ${ }_{\mathbb{R}} D^{*}$ so that $\Gamma$ acts discontinuously on $D^{*}$ and $\Gamma \backslash D^{*}$ is compact. It is clear that not all real boundary components need to be included in $D^{*}$ nor are desirable to be included. The following two points motivate the exact definition of $D^{*}$.

- $D^{*}$ should be big enough: By reduction theory, a fundamental domain $\Omega$ for the action of $\Gamma$ on $D$ may be formed from a finite union of Siegel sets $\mathfrak{S} \equiv C \widetilde{A}_{P_{0}}^{+} b \cdot x_{0}$. Here $P_{0}$ is a minimal parabolic $\mathbb{Q}$-subgroup, $C \subseteq P_{0}$ is compact, $b \in \widetilde{A}_{P_{0}}$, and $x_{0} \in D$ is a fixed basepoint. Call a real boundary component rationally visible if it occurs in the closure $\bar{\Omega} \subseteq{ }_{\mathbb{R}} D^{*}$ for some fundamental domain as above. For the quotient $\Gamma \backslash D^{*}$ to be compact, we 
wish to include in $D^{*}$ at least all rationally visible boundary components. One may check [25, Lemma 8.1] that the rationally visible boundary components are those whose normalizers $R$ contain a minimal parabolic $\mathbb{Q}$-subgroup and satisfy

$$
{ }_{\mathbb{R}} \Delta^{R}=\omega\left(\epsilon_{\mathbb{R} / \mathbb{Q}}(\Upsilon)\right) \quad \text { for some } \Upsilon \subseteq \Delta .
$$

- $D^{*}$ should not be too big: On the other hand, call a real boundary component geometrically rational if its normalizer $R$ is defined over $\mathbb{Q}$ and if there exists a normal subgroup $L_{R, \ell}^{\prime} \subseteq L_{R, \ell}$ defined over $\mathbb{Q}$ such that $L_{R, \ell} / L_{R, \ell}^{\prime}$ is compact. In this case, the decomposition in (22) can be modified to be defined over $\mathbb{Q}$, essentially by shifting certain compact factors from $L_{R, \ell}$ to $\widetilde{L}_{R, h}$; we denote these modified terms again by $L_{R, \ell}$ and $\widetilde{L}_{R, h}$. The geometrical rationality condition on a boundary component ensures that $\Gamma_{N_{R}}, \Gamma_{L_{R, \ell}} \equiv \Gamma_{L_{R}} \cap L_{R, \ell}$, and $\Gamma_{L_{R, h}} \equiv \Gamma_{L_{R}} / \Gamma_{L_{R, \ell}}$ are all arithmetic subgroups and hence act discontinuously on $N_{R}, D_{R, \ell}$, and $D_{R, h}$. To ensure that $\Gamma$ acts discontinuously on $D^{*}$, we do not want to include in $D^{*}$ boundary components that are not geometrically rational.

Any geometrically rational boundary component (in fact, any boundary component whose normalizer is defined over $\mathbb{Q}$ ) is rationally visible. The two points above suggest that we must assume conversely that

(iii) every rationally visible boundary component is geometrically rational.

A compactification $\mathbb{R}_{\mathbb{R}} D^{*}$ of $D$ satisfying this assumption is called geometrically rational. Under this assumption, the geometrically rational boundary components will simply be called rational boundary components and are just those with normalizers defined over $\mathbb{Q}$. We define

$$
D^{*} \equiv \coprod_{\begin{array}{c}
\text { rational } \\
\text { boundary components }
\end{array}} D_{R, h}
$$

Note that under the assumption of geometric rationality, the Levi quotient $L_{P}$ of any parabolic $\mathbb{Q}$-subgroup $P$ will have a decomposition (possibly different from (22) due to shifts of compact factors) into an almost direct product of reductive $\mathbb{Q}$-subgroups

$$
L_{P}=\widetilde{L}_{P, h} \times L_{P, \ell},
$$

where the $\mathbb{Q}$-root systems are $\kappa\left(\Delta^{P}\right)$ and $\zeta\left(\Delta^{P}\right)$ respectively. (The notation $\kappa\left(\Delta^{P}\right)$, $\zeta\left(\Delta^{P}\right)$, and $\omega\left(\Delta_{P}\right)$ is extended to subsets of $\Delta$ by using $\left.{ }_{\mathbb{Q}} \mu \equiv \mu\right|_{A}$ instead of $\mathbb{R}_{\mathbb{R}} \mu$.) The normalizers $R$ of the rational boundary components will be the saturated parabolic $\mathbb{Q}$-subgroups; these are those with $\omega\left(\Delta^{R}\right)=\Delta^{R}$.

The Satake compactification is known to be geometrically rational if $(\sigma, U)$ is strongly $\mathbb{Q}$-rational $[8]$ or if ${ }_{\mathbb{R}} D^{*}$ is the natural compactification of a Hermitian symmetric space $[4]$. In the latter case, as we have mentioned, all real boundary components are again Hermitian symmetric. Borel has suggested a natural generalization to consider. A real boundary component $D_{R, h}$ is equal-rank if $\mathbb{C}$-rank $L_{R, h}=\operatorname{rank} K_{R, h}$, where $K_{R, h}$ is a maximal compact subgroup of $L_{R, h}$. (A Hermitian symmetric space is automatically equal-rank.) Call $\mathbb{R}_{\mathbb{R}} D^{*}$ an real equalrank Satake compactification if $D$ and all its real boundary components are equalrank. Zucker [83, (A.2)] has enumerated the possible examples when $G$ is $\mathbb{R}$-simple; there are two new infinite families, $G=\mathrm{SO}(p, q)$ with $p+q$ odd and $G=\operatorname{Sp}(p, q)$, 
with $_{\mathbb{R}} \mu$ again connected to a simple $\mathbb{R}$-root at the end of the Dynkin diagram. In [64] we prove that a real equal-rank Satake compactification is geometrically rational, aside from some explicitly described $\mathbb{Q}$-rank 1 and 2 examples obtained by restriction of scalars.

8.5. The subspace topology on $D^{*} \subseteq_{\mathbb{R}} D^{*}$ is not the right one to use. Instead $D^{*}$ is given the Satake topology. To describe this topology, recall that $\bar{\Omega}$ is the closure in ${ }_{\mathbb{R}} D^{*}$ of a fundamental domain $\Omega$ constructed from Siegel sets. We have $D^{*}=\Gamma \cdot \bar{\Omega}$. Then a basis of local neighborhoods of $x \in D^{*}$ in the Satake topology is given by subsets $U$ such that

(i) If $x \in \gamma \bar{\Omega}$, then $U \cap \gamma \bar{\Omega}$ is open in the subspace topology on $\gamma \bar{\Omega} \subseteq{ }_{\mathbb{R}} D^{*}$.

(ii) $U$ is $\Gamma_{x}$-invariant, where $\Gamma_{x}=\{\gamma \in \Gamma \mid \gamma \cdot x=x\}$.

The Satake compactification $X^{*}$ is defined by

$$
X^{*} \equiv \Gamma \backslash D^{*}
$$

it has a stratification

$$
X^{*}=\coprod_{R \in \mathcal{P}^{*}} X_{R, h}
$$

where $\mathcal{P}^{*} \subseteq \mathcal{P}$ is the set of $\Gamma$-equivalence classes of saturated parabolic $\mathbb{Q}$-subgroups and

$$
X_{R, h} \equiv \Gamma_{L_{R, h}} \backslash D_{R, h} .
$$

The Satake compactification $X^{*}$ associated to the natural compactification of a Hermitian symmetric space is known as the Baily-Borel-Satake compactification; Baily and Borel [4] show that it admits the structure of a normal projective variety defined over a number field. In this case $\mathcal{P}^{*}$ simply consists of $\Gamma$-equivalence classes of maximal parabolic $\mathbb{Q}$-subgroups. More generally, the Satake compactification $X^{*}$ associated to a geometrically rational real equal-rank Satake compactification $D^{*}$ is again called a real equal-rank Satake compactification.

8.6. There is an alternative description due to Zucker [82] of the topology on $X^{*}$ that is quite useful. For $P \in \mathcal{P}$, we have the projection

$$
D_{P}=D_{P, h} \times D_{P, \ell} \longrightarrow D_{P, h}=D_{P^{\dagger}, h}
$$

onto the first factor of (23). This induces a map on the arithmetic quotients

$$
\pi_{P}: X_{P} \longrightarrow X_{P^{\dagger}, h}
$$

In general this is a flat bundle with typical fiber $X_{P, \ell} \equiv \Gamma_{L_{P, \ell}} \backslash D_{P, \ell}$, however if we replace $\Gamma_{L_{P}}$ by a certain finite index subgroup the corresponding bundle becomes trivial.

The maps $\pi_{P}$ for the various $P \in \mathcal{P}$ fit together to define a surjective map

$$
\pi: \widehat{X} \rightarrow X^{*}
$$

and Zucker proves that the topology on $X^{*}$ is the quotient topology induced by $\pi$. (See Figure 28 in $\S 19$ for a picture of the map $\pi$.) One can also define a continuous map $\widehat{D} \equiv \coprod_{P} D_{P} \rightarrow D^{*}$ before taking the arithmetic quotient, however this map is not necessarily open. 


\section{The Rapoport/Goresky-MacPherson conjecture}

The preceding sections, and especially the table at the end of $\S 5.3$, explicitly illustrate how the links of strata become more complicated as we pass from $\bar{X}$ to $\widehat{X}$ to $X^{*}$. The intersection cohomology sheaf will likewise become more complicated. A hope therefore is that one might be able to transfer the study of the cohomology of various sheaves, in particular the intersection cohomology sheaf, from $X^{*}$ to the simpler space $\widehat{X}$. (From now on in this paper, a "sheaf" means a complex of sheaves, viewed as an element of the constructible derived category.) In particular, the replacement of $X^{*}$ by $\widehat{X}$ in Theorem 3.1, Zucker's conjecture, would be of considerable use in applications.

For example, if $X^{*}$ is the Baily-Borel-Satake compactification of a Hermitian locally symmetric space, part of Langlands's program involves relating the HasseWeil zeta function of $X^{*}$ to automorphic $L$-functions; see for example [47] where Picard modular surfaces are considered. One approach to establishing this relationship would be to compare the Arthur-Selberg trace formula [2] for a Hecke operator on $H_{(2)}(X ; \mathbb{E})$ with a topological Lefschetz fixed point formula for $I_{p} H\left(X^{*} ; \mathbb{E}\right)$ such as Goresky and MacPherson give in [35]; this is reasonable since Zucker's conjecture shows that the groups are isomorphic. However Arthur's formula involves a sum over $P \in \mathcal{P}$ whereas a fixed point formula on $X^{*}$ will involve a sum over $R \in \mathcal{P}^{*}$; since $\mathcal{P}$ indexes the strata of $\widehat{X}$, a fixed point formula on $\widehat{X}$ might be more amenable to a comparison with Arthur's formula.

These considerations were one motivation for the following conjecture. It was first posed by Rapoport in 1986 within a letter to Borel [58]; the conjecture was later independently posed by Goresky and MacPherson and described in an unpublished preprint [34].

Conjecture 9.1 (Rapoport/Goresky-MacPherson). Let $X$ be a Hermitian locally symmetric space and let $p$ be a middle-perversity. There is a natural quasiisomorphism $\mathcal{I}_{p} \mathcal{C}\left(X^{*} ; \mathbb{E}\right) \cong \pi_{*} \mathcal{I}_{p} \mathcal{C}(\widehat{X} ; \mathbb{E})$ and hence $I_{p} H\left(X^{*} ; \mathbb{E}\right) \cong I_{p} H(\widehat{X} ; \mathbb{E})$.

Previously Zucker had noticed that the conjecture held for $G=\operatorname{Sp}_{4}(\mathbb{R})$ with $\mathbb{E}=\mathbb{C}$. In addition Goresky and MacPherson [34] announced the conjecture held for $G=\mathrm{Sp}_{4}(\mathbb{R}), \mathrm{Sp}_{6}(\mathbb{R})$, and (for $\mathbb{E}=\mathbb{C}$ ) $\operatorname{Sp}_{8}(\mathbb{R})$. In the case where $\mathbb{Q}$-rank $G=1$ a proof was given by the author and Stern in an appendix to a paper of Rapoport's [59]. The full conjecture (in fact a generalization to the equal-rank setting) was finally proved by the author in 2001 [61] (see Theorem 11.1 below).

\section{Weighted cohomology}

With similar motivation, but in a different direction from the above conjecture, Goresky, Harder, and MacPherson have defined the weighted cohomology $W^{\eta} H(\widehat{X} ; \mathbb{E})$ and have proven $[\mathbf{3 0}]$ :

Theorem 10.1 (Goresky-Harder-MacPherson). Let $X$ be a Hermitian locally symmetric space, let $p$ be a middle-perversity, and let $\eta$ be a middle-weight profile. There is a natural quasi-isomorphism $\mathcal{I}_{p} \mathcal{C}\left(X^{*} ; \mathbb{E}\right) \cong \pi_{*} \mathcal{W}^{\eta} \mathcal{C}(\widehat{X} ; \mathbb{E})$ and hence an isomorphism $I_{p} H\left(X^{*} ; \mathbb{E}\right) \cong W^{\eta} H(\widehat{X} ; \mathbb{E})$.

Weighted cohomology depends on a locally constant sheaf $\mathbb{E}$ on $X$ arising from a representation $E$ of $G$, as well as on a weight profile $\eta \in \mathfrak{a}_{P_{0}}^{G *}$ where $P_{0}$ is a minimal parabolic $\mathbb{Q}$-subgroup. The middle-weight profiles are $\nu=-\rho$ and $\mu=-\rho+\epsilon \rho$, 
where $\epsilon>0$ is very small and $\rho$ is one-half the sum of the positive $\mathbb{Q}$-roots of $G$ (counted with multiplicity). Like $L^{2}$-cohomology and intersection cohomology, there is a sheaf $\mathcal{W}^{\eta} \mathcal{C}(\widehat{X} ; \mathbb{E})$ whose hypercohomology is weighted cohomology.

We will give a local calculation of $W^{\eta} H(\widehat{X} ; \mathbb{E})$ analogous to the one for intersection cohomology in (8) of $\S 4.1$. Recall that the smooth part of the link of a stratum $X_{P} \subseteq \widehat{X}$ is $\mathcal{N}_{P}^{\prime} \times\left|\Delta_{P}\right|^{\circ}$ (see (21) in $\S 7$ ). Van Est's theorem [76] shows that the cohomology $H\left(\mathcal{N}_{P}^{\prime} \times\left|\Delta_{P}\right|^{\circ} ; \mathbb{E}\right) \cong H\left(\mathcal{N}_{P}^{\prime} ; \mathbb{E}\right)$ is naturally an $A_{P}^{G}$-module (in fact, an $L_{P}$-module); we will explain this in more detail in $\$ 12.1$. Thus there is a decomposition $H\left(\mathcal{N}_{P}^{\prime} ; \mathbb{E}\right)=\bigoplus_{\chi} H\left(\mathcal{N}_{P}^{\prime} ; \mathbb{E}\right)_{\chi}$ into $A_{P}^{G}$-weight spaces. If $U$ is a small neighborhood of $x \in X_{P}$, the local weighted cohomology $W^{\eta} H^{j}(U ; \mathbb{E})$ similarly has a decomposition into $A_{P}^{G}$-weight spaces and the local characterization is

$$
W^{\eta} H(U ; \mathbb{E})_{\chi} \cong \begin{cases}H\left(\mathcal{N}_{P}^{\prime} ; \mathbb{E}\right)_{\chi} & \text { for } \chi \geq \eta_{P}, \\ 0 & \text { for } \chi \geq \eta_{P} ;\end{cases}
$$

here $\eta_{P}$ is the restriction of $\eta$ to $\mathfrak{a}_{P}^{G}$ and the inequality $\chi \geq \eta_{P}$ means that $\chi-\eta_{P}$ is a nonnegative linear combination of elements of $\Delta_{P}$.

Remarks. (i) Despite the similarity of (25) with the local calculation of intersection cohomology (8), an important difference is that (25) is not inductive. Thus in general local calculations involving weighted cohomology are easier than the corresponding local calculations for intersection cohomology.

(ii) There is a natural projection $U \cap X \rightarrow \mathcal{N}_{P}^{\prime}$. If we represent a class in $H\left(\mathcal{N}_{P}^{\prime} ; \mathbb{E}\right)_{\chi} \cong H(U \cap X ; \mathbb{E})_{\chi}$ by a differential form $\phi$ which is the pullback of a form on $\mathcal{N}_{P}^{\prime}$, then the condition $\chi \geq \mu_{P}$ is precisely the condition that $\phi$ is $L^{2}$. Thus middle-weight profile weighted cohomology may be viewed as an algebraic analogue of $L^{2}$-cohomology.

Goresky and MacPherson [36] have applied their fixed point formula [35] to calculate the Lefschetz number of a Hecke correspondence on $W^{\eta} H(\widehat{X} ; \mathbb{E})$. They prove in collaboration with Kottwitz [31] that the result agrees with Arthur's trace formula $[\mathbf{2}]$ on $H_{(2)}(X ; \mathbb{E})$, in line with the motivation from the previous section.

A proof of Theorem 10.1 (different from the original one) can easily be sketched using Zucker's conjecture and later work of Nair [54]. First of all, Zucker's conjecture yields a quasi-isomorphism

$$
\mathcal{I}_{p} \mathcal{C}\left(X^{*} ; \mathbb{E}\right) \cong \mathcal{L}_{(2)}\left(X^{*} ; \mathbb{E}\right) .
$$

However it is fairly immediate that

$$
\mathcal{L}_{(2)}\left(X^{*} ; \mathbb{E}\right) \cong \pi_{*} \mathcal{L}_{(2)}(\widehat{X} ; \mathbb{E}) .
$$

Finally Nair's result is that there is a quasi-isomorphism

$$
\mathcal{L}_{(2)}(\widehat{X} ; \mathbb{E}) \cong \mathcal{W}^{\eta} \mathcal{C}(\widehat{X} ; \mathbb{E})
$$

as suggested by Remark (ii) above and hence

$$
\pi_{*} \mathcal{L}_{(2)}(\widehat{X} ; \mathbb{E}) \cong \pi_{*} \mathcal{W}^{\eta} \mathcal{C}(\widehat{X} ; \mathbb{E}) .
$$

Equations (26)-(28) yield Theorem 10.1. 


\section{Main theorem}

Despite the success of weighted cohomology, Conjecture 9.1 is still of interest. The short argument given above for Theorem 10.1 does not apply to prove the conjecture since in general the analogue of Nair's theorem does not hold: $\mathcal{I}_{p} \mathcal{C}(\widehat{X} ; \mathbb{E})$ is not quasi-isomorphic to $\mathcal{L}_{(2)}(\widehat{X} ; \mathbb{E})$ or $\mathcal{W}^{\eta} \mathcal{C}(\widehat{X} ; \mathbb{E})$ on $\widehat{X}$. The only hope for the conjecture is that a quasi-isomorphism exists downstairs on $X^{*}$ and indeed this is the case:

THEOREM 11.1. Let $X^{*}$ be a real equal-rank Satake compactification and let $p=$ $m$ or $n$ be a middle-perversity. There is a natural quasi-isomorphism $\mathcal{I}_{p} \mathcal{C}\left(X^{*} ; \mathbb{E}\right) \cong$ $\pi_{*} \mathcal{I}_{p} \mathcal{C}(\widehat{X} ; \mathbb{E})$ and hence an isomorphism $I_{p} H\left(X^{*} ; \mathbb{E}\right) \cong I_{p} H(\widehat{X} ; \mathbb{E})$.

This result is proved in $[\mathbf{6 1}]$ as a consequence of a number of results in the theory of $\mathcal{L}$-modules which we will outline in the remaining sections.

Note that the condition on $X^{*}$, that all real boundary components of ${ }_{\mathbb{R}} D^{*}$ are equal-rank, always holds if $D$ is Hermitian and $X^{*}$ is the Baily-Borel-Satake compactification. Thus the theorem implies the conjecture of Rapoport and GoreskyMacPherson. Note that although the equal-rank condition implies that the strata of $X^{*}$ have even dimension, the strata of $\widehat{X}$, on the other hand, may have odd codimension, even in the Hermitian case.

\section{L-modules}

In the course of work on the Rapoport/Goresky-MacPherson conjecture, it was realized that many of the techniques could be applied to study other sheaves. Furthermore, many parts of the proof, including a crucial and difficult local property of $\mathcal{I}_{p} \mathcal{C}(\widehat{X} ; \mathbb{E})$, apparently held even when $D$ was non-Hermitian or non-equal-rank. So it seemed desirable in view of other applications to separate the components of the proof and to work in a more general context. This was one motivation for us to develop the theory of $\mathcal{L}$-modules $[\mathbf{6 1}]$.

12.1. Overview. First recall the notation. The reductive Borel-Serre compactification has a stratification

$$
\widehat{X}=\coprod_{P \in \mathcal{P}} X_{P}
$$

where $\mathcal{P}$ is the partially ordered set of $\Gamma$-conjugacy classes of parabolic $\mathbb{Q}$-subgroups of $G$. For a parabolic $\mathbb{Q}$-subgroup $P$ of $G$, let $N_{P}$ denote its unipotent radical and let $L_{P}=P / N_{P}$ denote its Levi quotient. The reductive Borel-Serre boundary stratum $X_{P}$ is the locally symmetric space associated to $L_{P}$, namely

$$
X_{P}=\Gamma_{L_{P}} \backslash L_{P} / K_{P} A_{P}=\Gamma_{L_{P}} \backslash D_{P}
$$

where $A_{P}$ is the identity component of a maximal $\mathbb{Q}$-split torus in the center of $L_{P}, \Gamma_{P}=\Gamma \cap P, \Gamma_{N_{P}}=\Gamma \cap N_{P}$ and $\Gamma_{L_{P}}=\Gamma_{P} / \Gamma_{N_{P}}$. The link of $X_{P} \subseteq \widehat{X}$ has as its smooth open stratum $\mathcal{N}_{P}^{\prime} \times\left|\Delta_{P}\right|^{\circ}$, where $\mathcal{N}_{P}^{\prime}$ is a compact nilmanifold which is canonically diffeomorphic to $\Gamma_{N_{P}} \backslash N_{P}$ once a basepoint is chosen. The rest of the link is described in $\S 7$. In general, if $P \subseteq Q$, we extend all this notation to the parabolic $\mathbb{Q}$-subgroup $P / N_{Q}$ of $L_{Q}$ simply by adding a superscript $Q$; thus we have $N_{P}^{Q}=N_{P} / N_{Q}$, etc.

An $\mathcal{L}$-module

$$
\mathcal{M}=\left(E_{P}, f_{P Q}\right)_{P \leq Q \in \mathcal{P}}
$$


on $\widehat{X}$ is a combinatorial analogue of a constructible complex of sheaves. (The precise definition will be given below.) Like the constructible derived category of sheaves on $\widehat{X}$, the category of $\mathcal{L}$-modules has the functors $k_{*}, k_{!}, k^{*}$, and $k^{!}$as well as truncation functors. Furthermore there is a realization functor $\mathcal{M} \mapsto \mathcal{S}(\mathcal{M})$ from $\mathcal{L}$-modules to the constructible derived category of sheaves, commuting with these functors. Many familiar sheaves can be realized as $\mathcal{S}(\mathcal{M})$ (or "lifted to an $\mathcal{L}$ module"), for example, the intersection cohomology sheaf $\mathcal{I}_{p} \mathcal{C}(\widehat{X} ; \mathbb{E})$, the weighted cohomology sheaf $\mathcal{W}^{\eta} \mathcal{C}(\widehat{X} ; \mathbb{E})$, or the sheaf $i_{G *} \mathbb{E}$ whose hypercohomology is the ordinary cohomology $H(X ; \mathbb{E})$. The global cohomology of an $\mathcal{L}$-module is defined to be the hypercohomology of its realization:

$$
H(\widehat{X} ; \mathcal{M}) \equiv H(\widehat{X} ; \mathcal{S}(\mathcal{M})) .
$$

In fact the realization functor yields an explicit complex of fine sheaves so that the cohomology may be computed from the complex of their global sections; we will see this complex is easy to work with.

Let $i_{P}: X_{P} \hookrightarrow \widehat{X}$ and $\hat{\imath}_{P}: \widehat{X}_{P} \hookrightarrow \widehat{X}$ denote the inclusion of a stratum and of its closure. Recall that for a sheaf $\mathcal{S}$ to be in the constructible derived category, the local cohomology sheaf $H\left(i_{P}^{*} \mathcal{S}\right)$ along $X_{P}$ must be a finitely generated locally constant sheaf for all $P$. It is equivalent ${ }^{2}$ to require that the local cohomology $H\left(i_{P}^{!} \mathcal{S}\right)$ supported on $X_{P}$ is a finitely generated locally constant sheaf for all $P$; we prefer this point of view for reasons that will become clear later. Consequently for a sheaf, $H\left(i_{P}^{!} \mathcal{S}\right)$ may be identified with a graded representation of the arithmetic subgroup $\Gamma_{L_{P}} \subseteq L_{P}$. An important difference between a sheaf and an $\mathcal{L}$-module is that for an $\mathcal{L}$-module $\mathcal{M}, H\left(i_{P}^{!} \mathcal{M}\right)=H\left(E_{P}, f_{P P}\right)$ is actually a graded representation of the full reductive group $L_{P}$.

The reason this is possible, namely that the usual functorial operations applied to representations of Levi quotients yield representations of Levi quotients (as opposed to merely representations of fundamental groups) is van Est's theorem [76]. We will illustrate this with the simplest example of such a functorial operation. Suppose we start with a representation $E$ of $\Gamma \subseteq G$ and hence a locally constant sheaf $\mathbb{E}$ on $X=X_{G}$. Pushforward $\mathbb{E}$ to $i_{G *} \mathbb{E}$ on $\widehat{X}$, and then restrict it to $i_{P}^{*} i_{G *} \mathbb{E}$ on $X_{P}$. The local cohomology $H\left(i_{P}^{*} i_{G *} \mathbb{E}\right)$ is simply the cohomology of the smooth open stratum of the link $\mathcal{N}_{P}^{\prime} \times\left|\Delta_{P}\right|^{\circ}$, which is homotopic to $\mathcal{N}_{P}^{\prime}$. Thus the local cohomology is $H\left(\mathcal{N}_{P}^{\prime} ; \mathbb{E}\right)$ and since this is the cohomology of the fiber of the flat bundle $Y_{P} \rightarrow X_{P}$, it has an action on it of $\Gamma_{L_{P}} \subseteq L_{P}$. However van Est's theorem says that if $E$ actually arose from a representation of $G$, there is an isomorphism of $\Gamma_{L_{P}}$-modules

$$
H\left(\mathcal{N}_{P}^{\prime} ; \mathbb{E}\right) \cong H\left(\mathfrak{n}_{P} ; E\right) .
$$

The $\Gamma_{L_{P}}$-action on the complex $\bigwedge \mathfrak{n}_{P}^{*} \otimes E$ is obtained by lifting $\Gamma_{L_{P}}$ to $G$; the induced action on the cohomology $H\left(\mathfrak{n}_{P} ; E\right)$ is independent of the lift. This $\Gamma_{L_{P}}{ }^{-}$ action, however, extends to an $L_{P}$-action defined by the same procedure.

The idea of representing a sheaf by combinatorial and linear algebraic data is not new. In other contexts there is the theory of $S$-constructible sheaves [41], [42, Exercise VIII.1] and MacPherson's theory of cellular sheaves [70], [78], [79]. These two examples are based on a decomposition into contractible cells, unlike the decomposition of $\widehat{X}$ into strata. For examples with non-contractible cells, there is

\footnotetext{
${ }^{2}$ This follows by the methods of $[\mathbf{1 5}, \mathrm{V} .3]$ though it is not explicitly stated there.
} 
the theory of sheaves on fans $[\mathbf{5}],[\mathbf{6}],[\mathbf{2 2}],[\mathbf{2 3}],[\mathbf{5 2}]$ and Braden and MacPherson's moment graphs $[\mathbf{2 1}]$. There is also forthcoming work of Braden [20] on Koszul duality, in which he constructs a combinatorial category of mixed sheaves on affine toric varieties. The forthcoming work of Pink and Wildeshaus on automorphic sheaves $[\mathbf{8 0}]$ applies in a context closest to that of $\mathcal{L}$-modules. The basic linear algebraic data in all these theories is the local cohomology along a cell or stratum, $i_{P}^{*} \mathcal{S}$. An $\mathcal{L}$-module uses instead the dual notion of the local cohomology supported on a stratum $E_{P}=i_{P}^{!} \mathcal{S}$ as the building block. This approach turns out to make the construction of a useful realization technically easier.

12.2. L-modules. An $\mathcal{L}$-module $\mathcal{M}=(E$., f.. $)$ on $\widehat{X}$ consists of

- for all $P \in \mathcal{P}$, a graded algebraic representation $E_{P}$ of $L_{P}$;

- for all $P \leq Q$, morphisms $f_{P Q}: H\left(\mathfrak{n}_{P}^{Q} ; E_{Q}\right) \stackrel{[1]}{\longrightarrow} E_{P}$.

(The superscript [1] indicates that $f_{P Q}$ is graded of degree 1, that is, it maps $H^{i}\left(\mathfrak{n}_{P}^{Q} ; E_{Q}\right)$ into $E_{P}^{i+1}$.) This data must satisfy the condition that for all $P \leq R$,

$$
\sum_{P \leq Q \leq R} f_{P Q} \circ H\left(\mathfrak{n}_{P}^{Q} ; f_{Q R}\right)=0 .
$$

In particular, $\left(E_{P}, f_{P P}\right)$ is a complex of $L_{P}$-modules. Here $H\left(\mathfrak{n}_{P}^{Q} ; f_{Q R}\right)$ is viewed as a map $H\left(\mathfrak{n}_{P}^{R} ; E_{R}\right) \rightarrow H\left(\mathfrak{n}_{P}^{Q} ; E_{Q}\right)$ via the canonical isomorphism

$$
H\left(\mathfrak{n}_{P}^{R} ; E_{R}\right) \cong H\left(\mathfrak{n}_{P}^{Q} ; H\left(\mathfrak{n}_{Q}^{R} ; E_{R}\right)\right) .
$$

This is a good place to recall the standard notation regarding shifts of degree: if $C$ is a complex, then $C[k]$ denotes the shifted complex defined by

$$
C[k]^{i} \equiv C^{i+k}, \quad d_{C[k]} \equiv(-1)^{k} d_{C} .
$$

This applies to graded objects (viewed as complexes with zero differential) and ordinary objects (viewed as complexes with nonzero entries only in degree 0 ) as well. Thus if $P<Q$ and there are no elements of $\mathcal{P}$ strictly between $P$ and $Q$, the condition (30) shows that $f_{P Q}$ is a morphism of complexes,

$$
\left(H\left(\mathfrak{n}_{P}^{Q} ; E_{Q}\right), H\left(\mathfrak{n}_{P}^{Q} ; f_{Q Q}\right)\right) \longrightarrow\left(E_{P}, f_{P P}\right)[1] .
$$

A morphism $\phi=(\phi .$.$) of \mathcal{L}$-modules $\mathcal{M} \rightarrow \mathcal{M}^{\prime}$ consists of graded maps

$$
\phi_{P Q}: H\left(\mathfrak{n}_{P}^{Q} ; E_{Q}\right) \longrightarrow E_{P}^{\prime} \quad \text { for all } P \leq Q
$$

satisfying

$$
\sum_{P \leq Q \leq R} \phi_{P Q} \circ H\left(\mathfrak{n}_{P}^{Q} ; f_{Q R}\right)=\sum_{P \leq Q \leq R} f_{P Q}^{\prime} \circ H\left(\mathfrak{n}_{P}^{Q} ; \phi_{Q R}\right) \quad \text { for all } P \leq R .
$$

A subspace $W \subseteq \widehat{X}$ is admissible if it is a locally closed union of strata; set $\mathcal{P}(W)=\left\{P \in \mathcal{P} \mid X_{P} \subseteq W\right\}$. In this case we define an $\mathcal{L}$-module on $W$ as above but with $P, Q$, and $R$ restricted to belong to $\mathcal{P}(W)$.

To illustrate the above definition, suppose that $\mathbb{Q}-\operatorname{rank} G=2$ and that there is only one $\Gamma$-conjugacy class of parabolic $\mathbb{Q}$-subgroups of each type; an example where this occurs is $G=\operatorname{Sp}_{4}(\mathbb{R})$ and $\Gamma=\operatorname{Sp}_{4}(\mathbb{Z})$. Then $\mathcal{P}=\left\{P, Q_{1}, Q_{2}, G\right\}$ with the covering relations $P<Q_{1}, Q_{2}<G$. In Figure 8 we represent $\mathcal{P}$ by its Hasse diagram (which we invert so as to match with Figure 6). The data of an $\mathcal{L}$-module $\mathcal{M}$ for this example (but not condition (30)) is displayed in Figure 9. 


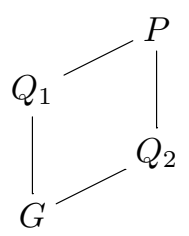

Figure 8. The (inverted) Hasse diagram for $\mathcal{P}=\left\{P<Q_{1}, Q_{2}<G\right\}$

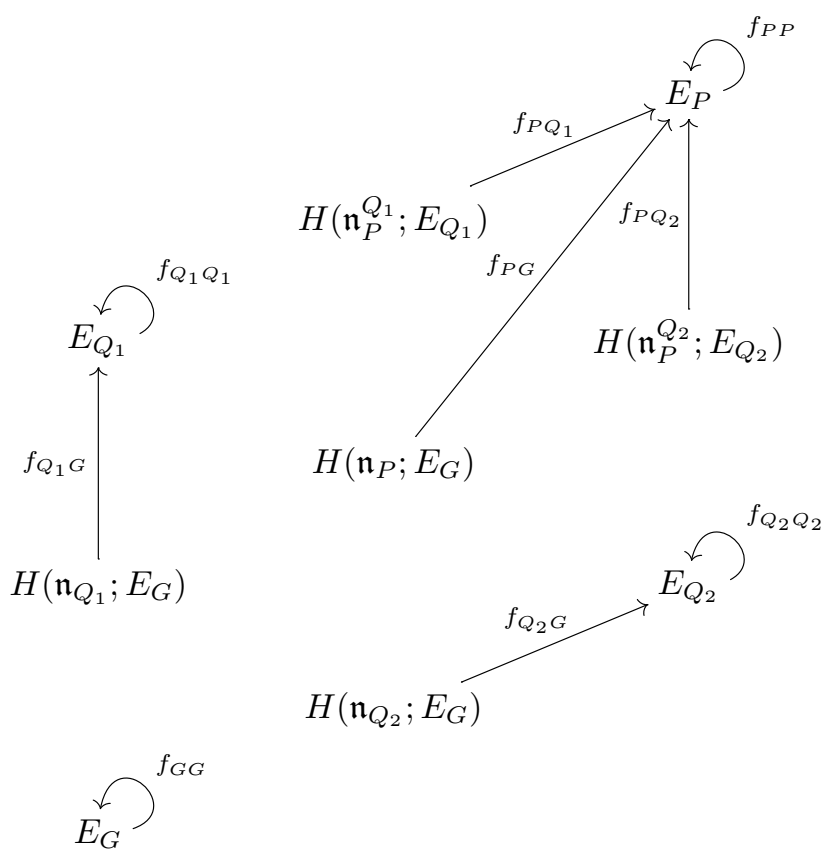

Figure 9 . The data of a typical $\mathcal{L}$-module $\mathcal{M}$ for the $\mathcal{P}$ from

Figure 8. (All objects are graded and all morphisms are degree 1.)

12.3. Functors on $\mathcal{L}$-modules. We will only define the usual functors in the cases we need here. If $k: Z \hookrightarrow W$ is an inclusion of admissible spaces, then $k ! \mathcal{M}$ for an $\mathcal{L}$-module $\mathcal{M}$ on $W$ is defined by restricting the subscripts of $E_{P}$ and $f_{P Q}$ to belong to $\mathcal{P}(Z)$. In particular,

$$
i_{P}^{!} \mathcal{M}=\left(E_{P}, f_{P P}\right)
$$

for all $P \in \mathcal{P}(W)$. As we have noted, this is a complex of $L_{P}$-modules; its cohomology $H\left(i_{P} \mathcal{M}\right)$ is the local cohomology supported on $X_{P}$. The less trivial example of $\hat{\imath}_{Q_{1}}^{!} \mathcal{M}$ for our example is represented in Figure 10.

On the other hand, for $P \in \mathcal{P}(W)$ we define

$$
i_{P}^{*} \mathcal{M} \equiv\left(\bigoplus_{P \leq Q} H\left(\mathfrak{n}_{P}^{Q} ; E_{Q}\right), \sum_{P \leq Q \leq R} H\left(\mathfrak{n}_{Q}^{R} ; f_{Q R}\right)\right) .
$$




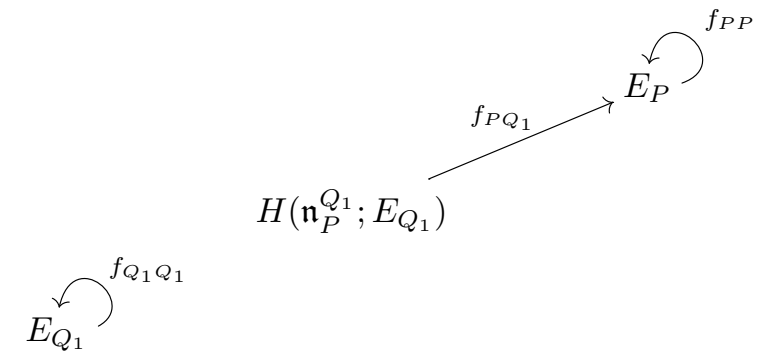

Figure 10. The $\mathcal{L}$-module $\hat{\imath}_{Q_{1}}^{!} \mathcal{M}(\mathcal{M}$ as in Figure 9$)$

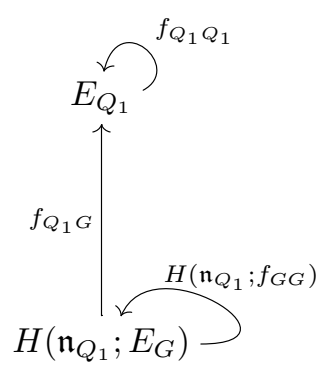

(a) $i_{Q_{1}}^{*} \mathcal{M}$

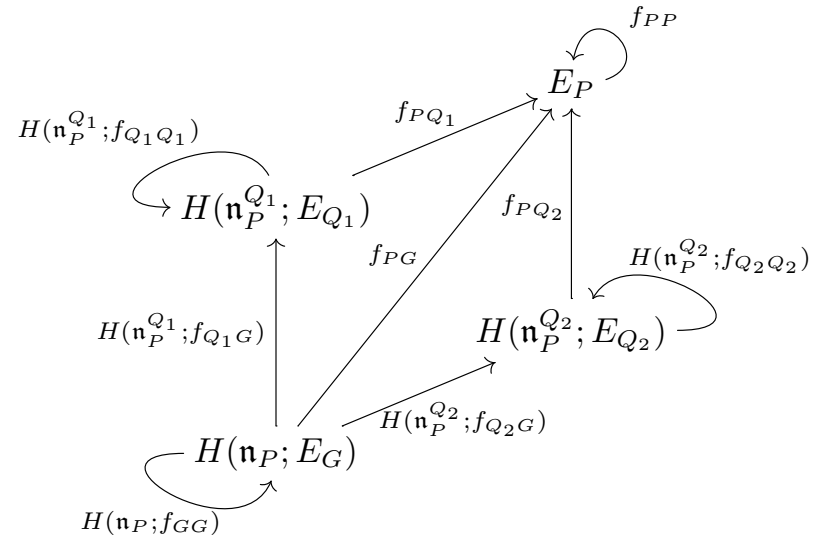

(b) $i_{P}^{*} \mathcal{M}$

Figure 11. The complexes computing local cohomology of $\mathcal{M}$ at $Q_{1}$ and at $P(\mathcal{M}$ as in Figure 9$)$

Again by (30) this is a complex of $L_{P}$-modules; its cohomology $H\left(i_{P}^{*} \mathcal{M}\right)$ is the local cohomology at $X_{P}$ (or at $P$ ). The complexes $i_{Q_{1}}^{*} \mathcal{M}$ and $i_{P}^{*} \mathcal{M}$ for our usual example are pictured in Figure 11.

If $R \in \mathcal{P}(W)$, the pullback $\hat{\imath}_{R}^{*} \mathcal{M}=\left(E_{.}^{\prime}, f_{\prime .}^{\prime}\right)$ to an entire closed face $\widehat{X}_{R} \cap W$ is defined by

$$
E_{P}^{\prime} \equiv \bigoplus_{\substack{Q \geq P \\ Q \cap \bar{R}=P}} H\left(\mathfrak{n}_{P}^{Q} ; E_{Q}\right) \quad \text { for all } P \leq R,
$$

with the obvious induced morphisms for $f_{\text {... }}^{\prime}$.

Finally $k_{*} \mathcal{M}^{\prime}$ for an $\mathcal{L}$-module $\mathcal{M}^{\prime}$ on $Z$ is defined by extending $E_{P}$ and $f_{P Q}$ to be zero if $P$ or $Q$ belongs to $\mathcal{P}(W) \backslash \mathcal{P}(Z)$.

In the cases we have defined, the usual adjoint relations hold among these functors. (For some of the other cases, it may be necessary to pass to the "homotopy category of $\mathcal{L}$-modules" which we do not consider here.) In particular, $i_{P}^{*}$ is a left adjoint to $i_{P *}$ on $\mathcal{L}$-modules and so there is a natural adjunction morphism

$$
\mathcal{M} \longrightarrow i_{P *} i_{P}^{*} \mathcal{M}
$$




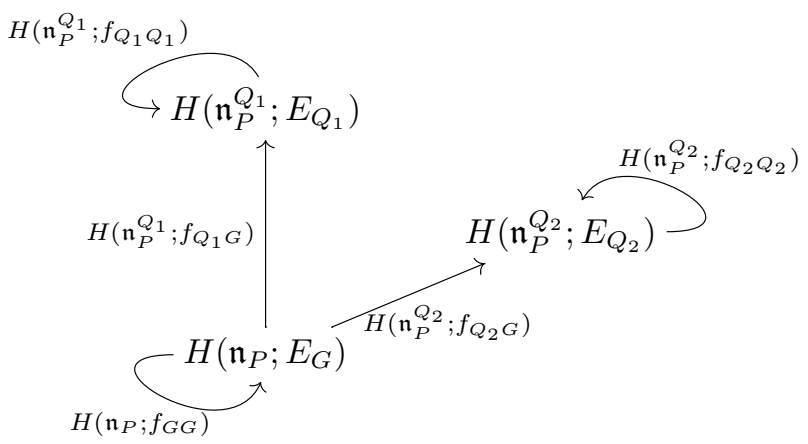

Figure 12. The complex computing the link cohomology of $\mathcal{M}$ at $P(\mathcal{M}$ as in Figure 9$)$

which is characterized by the condition that $i_{P}^{*} \mathcal{M} \longrightarrow i_{P}^{*} i_{P *} i_{P}^{*} \mathcal{M}=i_{P}^{*} \mathcal{M}$ is the identity map.

Besides $i_{P *} i_{P}^{*} \mathcal{M}$, certain other compositions of these functors are important. For example, let $j_{P}: W \backslash X_{P} \hookrightarrow W$ denote the inclusion of the complement of $X_{P}$. The functor $i_{P}^{*} j_{P *} j_{P}^{*} \mathcal{M}$ is a complex of $L_{P}$-modules whose cohomology is the link cohomology of $\mathcal{M}$ at $P$. An example is pictured in Figure 12.

The composed functor $i_{P}^{*} \hat{l}_{Q} \mathcal{M}$, where $P \leq Q$, will be important in $\S 13$ when we define the micro-support of an $\mathcal{L}$-module. Here are some examples. Since $\hat{\imath}_{G}^{!}$is the identity, Figure 11 already gives two examples in the case $Q=G$. The other extreme is $Q=P$; in this case $i_{P}^{*} \hat{\imath}_{Q} \mathcal{M}=\hat{\imath}_{P}^{!} \mathcal{M}=\left(E_{P}, f_{P P}\right)$. For an example with $P<Q<G$, consider

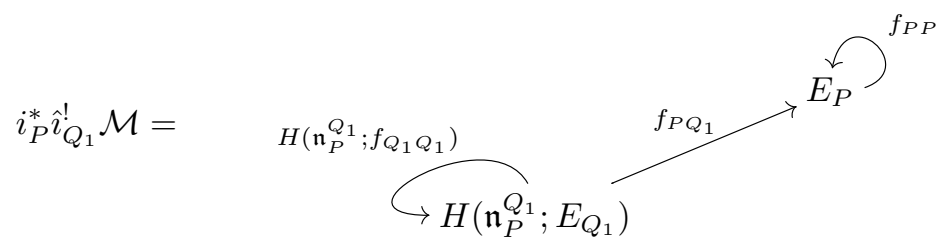

where $\mathcal{M}$ is as in Figure 9.

12.4. Realization of $\mathcal{L}$-modules. In order to define the realization sheaf of an $\mathcal{L}$-module, we need to recall the notion of special differential forms. The sheaf of special differential forms $\mathcal{A}_{\mathrm{sp}}(\widehat{X} ; \mathbb{E})$ on $\widehat{X}$ for a $G$-module $E$ was introduced by Goresky, Harder, and MacPherson [30]. It is a fine resolution of $i_{G *} \mathbb{E}$ whose sections on $U \subseteq \widehat{X}$ are smooth differential forms on the inverse image $\widetilde{U} \subseteq \bar{X}$ with coefficients in $\mathbb{E}$ which satisfy certain boundary conditions. Namely, in a neighborhood $\widetilde{U}$ of a boundary point in $Y_{P}$, the forms are locally pullbacks of forms on $Y_{P}$ under the geodesic retraction $\widetilde{U}=B \times c\left(\left|\Delta_{P}\right|\right) \rightarrow B \subseteq Y_{P}$. Furthermore these boundary values are required to be constant along the nilmanifold fibers $\mathcal{N}_{P}^{\prime}$. In the case that $E=\bigoplus_{i} E^{i}[-i]$ is graded, the differential $d$ is the alternating sum of the corresponding de Rham exterior differentials. One may show, essentially by applying harmonic projection along the nilmanifold fibers and using van Est's 


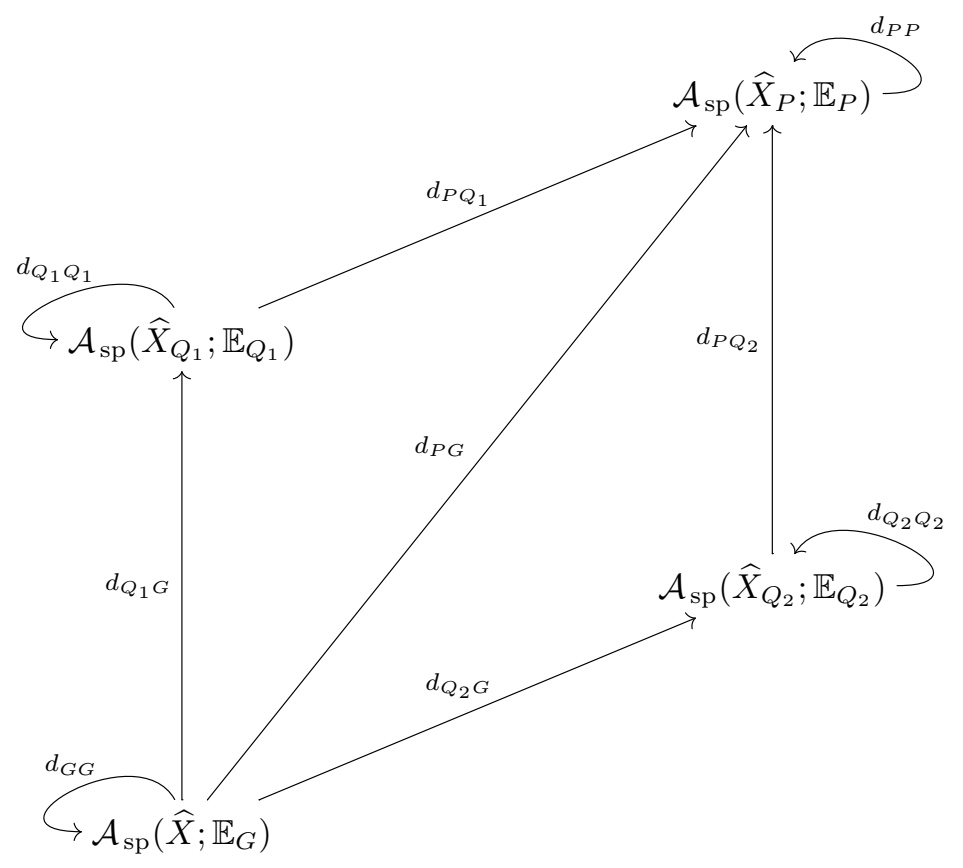

FiguRE 13. The realization sheaf $\mathcal{S}(\mathcal{M})(\mathcal{M}$ as in Figure 9$)$

theorem (29), that there is a natural morphism, "restriction to a stratum",

$$
k_{P}: \mathcal{A}_{\mathrm{sp}}(\widehat{X} ; \mathbb{E}) \longrightarrow \hat{\imath}_{P *} \mathcal{A}_{\mathrm{sp}}\left(\widehat{X}_{P} ; \mathbb{H}\left(\mathfrak{n}_{P} ; E\right)\right)
$$

which restricts over $\widehat{X}_{P}$ to be a quasi-isomorphism. Given a graded $L_{Q}$-module $E_{Q}$ we may likewise define the sheaf of special differential forms $\mathcal{A}_{\mathrm{sp}}\left(\widehat{X}_{Q} ; \mathbb{E}_{Q}\right)$, a differential $d_{Q}$, and a natural morphism

$$
k_{P Q}: \mathcal{A}_{\mathrm{sp}}\left(\widehat{X}_{Q} ; \mathbb{E}_{Q}\right) \longrightarrow \hat{\imath}_{P *} \mathcal{A}_{\mathrm{sp}}\left(\widehat{X}_{P} ; \mathbb{H}\left(\mathfrak{n}_{P}^{Q} ; E_{Q}\right)\right) .
$$

The realization $\mathcal{S}(\mathcal{M})$ of an $\mathcal{L}$-module $\mathcal{M}$ on $\widehat{X}$ can now be defined to be the complex of sheaves

$$
\left(\bigoplus_{P \in \mathcal{P}} \hat{\imath}_{P *} \mathcal{A}_{\mathrm{sp}}\left(\widehat{X}_{P} ; \mathbb{E}_{P}\right), \sum_{P \leq Q \in \mathcal{P}} d_{P Q}\right)
$$

where

$$
d_{P Q}= \begin{cases}d_{P}+\mathcal{A}_{\mathrm{sp}}\left(\widehat{X}_{P} ; f_{P P}\right) & \text { if } P=Q \\ \mathcal{A}_{\mathrm{sp}}\left(\widehat{X}_{P} ; f_{P Q}\right) \circ k_{P Q} & \text { if } P<Q .\end{cases}
$$

The realization sheaf $\mathcal{S}(\mathcal{M})$ for $\mathcal{M}$ as in Figure 9 is pictured in Figure 13. (We do not show the factorization of the arrows however.)

It is common to compute the cohomology of a sheaf by a complex consisting of differential forms satisfying boundary conditions or growth conditions depending on the particular sheaf. In view of the definition of $\mathcal{S}(\mathcal{M})$, however, we have a uniform type of complex to compute the cohomology of an $\mathcal{L}$-module: special differential forms with coefficients. The complication is that these "forms" are 
actually collections of forms on $\widehat{X}$ and on the various strata $\widehat{X}_{P}$, and there are interaction terms obtained by restricting to boundary strata and applying $f_{P Q}$. The boundary conditions mentioned above are translated into the choice of the coefficients and the $f_{P Q}$; see the example of intersection cohomology below.

It is easy to check that the realization functor commutes with the usual functors in the cases we have defined them, that is, there are natural quasi-isomorphisms $\mathcal{S}\left(k^{!} \mathcal{M}\right) \cong k ! \mathcal{S}(\mathcal{M})$, etc.

12.5. Reduced $\mathcal{L}$-modules. An $\mathcal{L}$-module $\mathcal{M}=(E$., f.. $)$ is called reduced if $f_{P P}=0$ for all $P$. One can show that for any $\mathcal{L}$-module $\mathcal{M}$, there exists a reduced $\mathcal{L}$-module $\mathcal{M}_{0}$ which is quasi-isomorphic to $\mathcal{M}$; quasi-isomorphic here means that there exists a third $\mathcal{L}$-module $\mathcal{M}^{\prime}$ and morphisms $\mathcal{M} \leftarrow \mathcal{M}^{\prime} \rightarrow \mathcal{M}_{0}$ which both induce isomorphisms on local cohomology. It follows that $H(\widehat{X} ; \mathcal{M}) \cong H\left(\widehat{X} ; \mathcal{M}_{0}\right)$. One could develop the theory by requiring $f_{P P}=0$ in the definition of an $\mathcal{L}$ modules to begin with, however in that case the definitions of the usual functors, in particular the truncation functors defined below, would be more complicated.

12.6. Simplest example. The simplest sheaf that can be lifted to an $\mathcal{L}$ module is $i_{G *} \mathbb{E}$, where $E$ is a $G$-module in degree 0 ; the cohomology is $H\left(\widehat{X} ; i_{G *} \mathbb{E}\right) \cong$ $H(X ; \mathbb{E}) \cong H(\Gamma ; E)$. The lifted $\mathcal{L}$-module $i_{G *} E$ is defined by

$$
\begin{aligned}
E_{P} & \equiv \begin{cases}E & \text { if } P=G, \\
0 & \text { if } P \neq G,\end{cases} \\
f_{P Q} & \equiv 0 .
\end{aligned}
$$

The local cohomology at $X_{P}$ is simply $i_{P}^{*} i_{G *} E=H\left(\mathfrak{n}_{P} ; E\right)$.

12.7. Truncation functors. Recall that the formula for the intersection cohomology sheaf (9) involves the functor $\tau^{\leqslant n}$ which truncates local cohomology in degrees greater than $n$ :

$$
\tau^{\leqslant n} \mathcal{S} \equiv \quad \cdots \stackrel{d_{n-2}}{\longrightarrow} \mathcal{S}^{n-1} \stackrel{d_{n-1}}{\longrightarrow} \operatorname{ker} d_{n} \stackrel{d_{n}}{\longrightarrow} 0 \longrightarrow \cdots .
$$

In order to lift intersection cohomology to an $\mathcal{L}$-module, one needs an analogue of this truncation functor for $\mathcal{L}$-modules.

Consider the simplest $\mathcal{L}$-module $i_{G *} E$ on the simplest nontrivial $\widehat{X}$ where there is only one singular stratum corresponding to the parabolic subgroup $P$. We assume $E$ is purely in degree 0 and $n \geq 0$. What should the $\mathcal{L}$-module $\tau^{\leqslant n} i_{G *} E$ be? The data $E_{G}=E$ cannot be changed; instead one must define $E_{P}$ and $f_{P G}$ so that the local cohomology $H\left(i_{P}^{*} \tau^{\leqslant n} i_{G *} E\right)$ at $X_{P}$ changes from $H\left(\mathfrak{n}_{P} ; E\right)$ to $\bigoplus_{i \leq n} H^{i}\left(\mathfrak{n}_{P} ; E\right)[-i]$. Since the pullback $i_{P}^{*} \tau^{\leqslant n} i_{G *} E$ is

$$
H\left(\mathfrak{n}_{P} ; E\right) \stackrel{f_{P G}}{\longrightarrow} E_{P}
$$

and there is a natural decomposition

$$
H\left(\mathfrak{n}_{P} ; E\right)=\left(\bigoplus_{i \leq n} H^{i}\left(\mathfrak{n}_{P} ; E\right)[-i]\right) \oplus\left(\bigoplus_{i>n} H^{i}\left(\mathfrak{n}_{P} ; E\right)[-i]\right),
$$

the desired truncation of local cohomology can be arranged by setting

$$
E_{P}=\left(\bigoplus_{i>n} H^{i}\left(\mathfrak{n}_{P} ; E\right)[-i]\right)[-1]
$$


and letting $f_{P G}$ be the natural degree 1 map (projection onto the second factor of $(34)$ ).

In essence the "internal" truncation functor $\tau^{\leqslant n}(33)$ has been realized as an "external" truncation functor. McConnell does something similar with his "flabby truncation" functor $\check{\tau}_{\leqslant n}$ [52]. That this is possible is not surprising. Recall that for a map of complexes $f:\left(C, d_{C}\right) \rightarrow\left(D, d_{D}\right)$ (where the objects lie in any additive category), the mapping cone $M(f)$ is the complex defined by

$$
\left(C[1] \oplus D,\left(\begin{array}{cc}
d_{C[1]} & 0 \\
f & d_{D}
\end{array}\right)\right)
$$

The truncation $\tau^{\leqslant n} \mathcal{S}$ of a sheaf is quasi-isomorphic to the mapping cone

$$
M\left(\mathcal{S} \rightarrow \tau^{>n} \mathcal{S}\right)[-1],
$$

where

$$
\tau^{>n} \mathcal{S} \equiv \quad \cdots 0 \longrightarrow \mathcal{S}^{n+1} / \operatorname{Im} d_{n} \stackrel{d_{n+1}}{\longrightarrow} \mathcal{S}^{n+2} \stackrel{d_{n+2}}{\longrightarrow} \cdots
$$

and

$$
\mathcal{S} \rightarrow \tau^{>n} \mathcal{S}
$$

is the natural map. With this notation, equation (35) may be re-expressed as

$$
E_{P}=\tau^{>n} H\left(\mathfrak{n}_{P} ; E\right)[-1]
$$

(the degree shift is applied after the truncation).

In order to define $\tau^{\leqslant n} \mathcal{M}$ for a general $\mathcal{L}$-module $\mathcal{M}$, we proceed stratum by stratum. Namely assume that for a fixed stratum $X_{P}$, the local cohomology of $\mathcal{M}$ at all $X_{Q}$ with $Q>P$ already vanishes in degrees greater than $n$. There is an obvious notion of a mapping cone for a morphism of $\mathcal{L}$-modules and to truncate the local cohomology at $P$ we define

$$
\tau_{P}^{\leqslant n} \mathcal{M} \equiv M\left(\mathcal{M} \rightarrow i_{P *} \tau^{>n} i_{P}^{*} \mathcal{M}\right)[-1] .
$$

The morphism here is the composition

$$
\mathcal{M} \longrightarrow i_{P *} i_{P}^{*} \mathcal{M} \longrightarrow i_{P *} \tau^{>n} i_{P}^{*} \mathcal{M}
$$

of the adjunction morphism with one induced from (37). An example of $\tau_{P}^{\leqslant n}$ where $P$ has parabolic rank 2 will be given in the next subsection. The functor $\tau^{\leqslant n}$ is defined to be the composition of all the functors $\tau_{P}^{\leqslant n}$, where one applies $\tau_{R}^{\leqslant n}$ before $\tau_{Q}^{\leqslant n}$ if $Q<R$.

It is easy to check that there is a natural quasi-isomorphism

$$
\mathcal{S}\left(\tau^{\leqslant n} \mathcal{M}\right) \cong \tau^{\leqslant n} \mathcal{S}(\mathcal{M}) .
$$

12.8. Intersection cohomology. The formula (9) for the intersection cohomology sheaf $\mathcal{I}_{p} \mathcal{C}(\widehat{X} ; \mathbb{E})$ involves pushforward and truncation; since both of these functors have been defined for $\mathcal{L}$-modules, commuting with the realization functor, we can lift intersection cohomology to an $\mathcal{L}$-module $\mathcal{I}_{p} \mathcal{C}(\widehat{X} ; E)$. Specifically, enumerate the elements of $\mathcal{P}$ as $P_{0}=G, P_{1}, \ldots, P_{N}$ such that $\operatorname{dim} X_{P_{i}} \geq \operatorname{dim} X_{P_{j}}$ for $i<j$. For $k<N$, let

$$
j_{P_{k}}: \coprod_{i<k} X_{P_{i}} \hookrightarrow \coprod_{i \leq k} X_{P_{i}}
$$



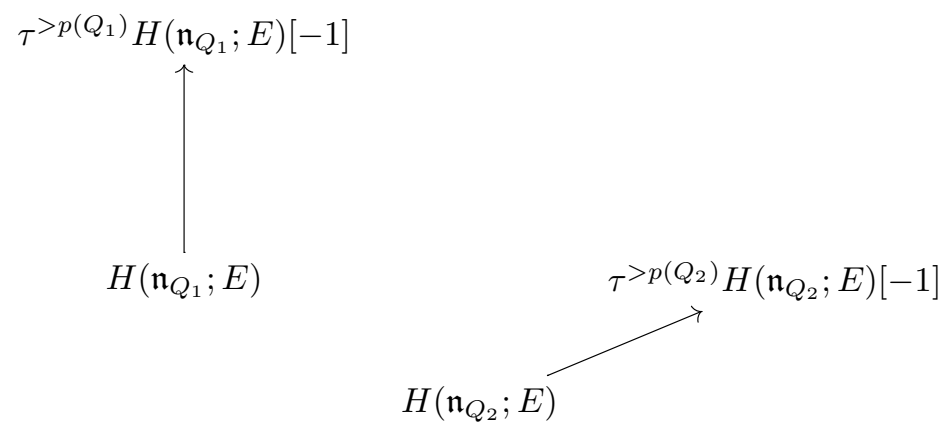

$E$

Figure 14. The $\mathcal{L}$-module $\mathcal{I}_{p} \mathcal{C}\left(X \sqcup X_{Q_{1}} \sqcup X_{Q_{2}} ; E\right)$. (The unlabeled morphisms are the natural degree 1 maps.)

be the inclusion map. Define

$$
p(Q) \equiv p\left(\operatorname{dim} \mathfrak{n}_{Q}+\# \Delta_{Q}\right) \quad \text { for } Q \in \mathcal{P} ;
$$

this is reasonable since $\operatorname{dim} \mathfrak{n}_{Q}+\# \Delta_{Q}$ is the codimension of $X_{Q}$ in $\widehat{X}$ (namely 1 more than the dimension of the link (21)). The intersection cohomology $\mathcal{L}$-module is defined as

$$
\mathcal{I}_{p} \mathcal{C}(\widehat{X} ; E) \equiv \tau^{\leqslant p\left(P_{N}\right)} j_{P_{N} *} \cdots \tau^{\leqslant p\left(P_{2}\right)} j_{P_{2} *} \leqslant p\left(P_{1}\right) j_{P_{1} *} E,
$$

where the $G$-module $E$ is viewed as usual as an $\mathcal{L}$-module on $X=X_{G}$.

Let us illustrate this procedure for our standard $\mathbb{Q}$-rank 2 example with $\mathcal{P}$ as in Figure 8. Obtaining the $\mathcal{L}$-module over $X_{G} \sqcup X_{Q_{1}} \sqcup X_{Q_{2}}$ is straightforward; the result is pictured in Figure 14. The complex $i_{P}^{*} j_{P *} \mathcal{I}_{p} \mathcal{C}\left(X \sqcup X_{Q_{1}} \sqcup X_{Q_{2}} ; E\right)$ computing the intersection cohomology of the link at $P$ is then

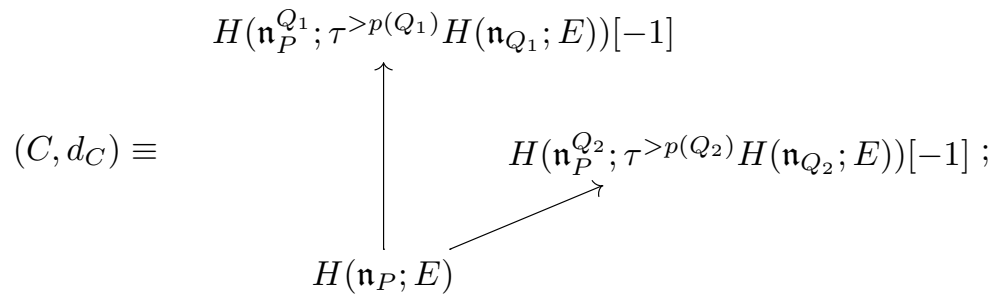

the final $\mathcal{L}$-module $\mathcal{I}_{p} \mathcal{C}(\widehat{X} ; E)$ is pictured in Figure 15.

\section{Micro-support of an $\mathcal{L}$-module}

A key local invariant of an $\mathcal{L}$-module defined in [61] is its micro-support; the terminology reflects the rough analogy that exists between this concept and that introduced by Kashiwara and Schapira [42] for sheaves. The micro-support $\operatorname{SS}(\mathcal{M})$ of an $\mathcal{L}$-module $\mathcal{M}$ is a certain set of irreducible representations $V$ of the various $L_{P}$ which we now define. 


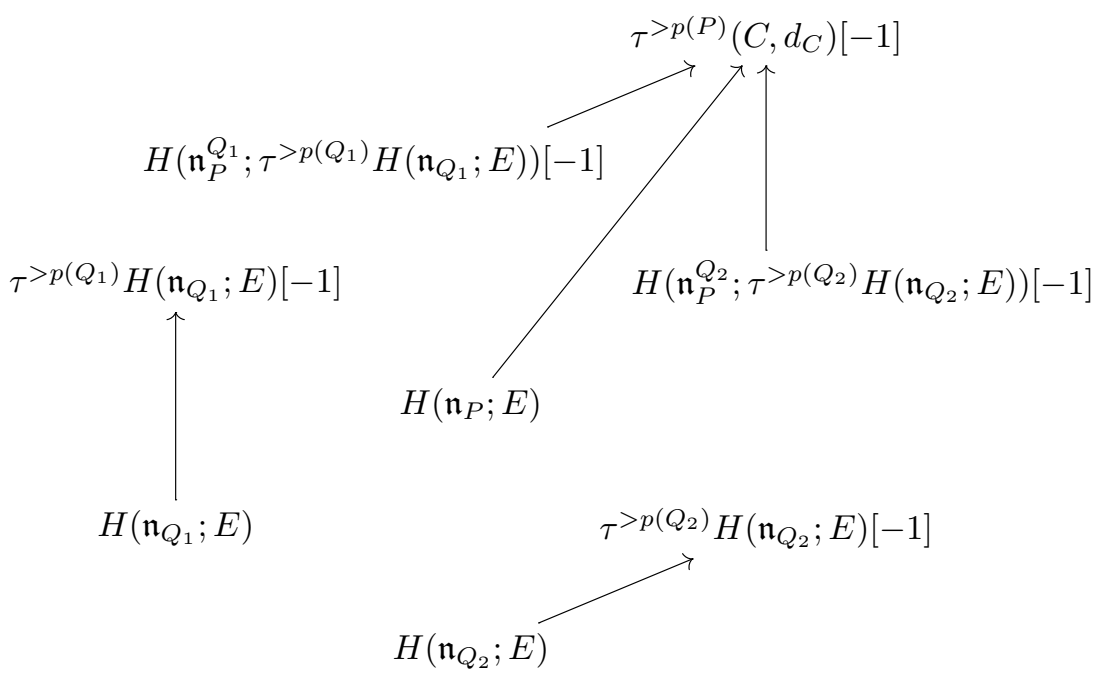

$E$

Figure 15 . The $\mathcal{L}$-module $\mathcal{I}_{p} \mathcal{C}(\widehat{X} ; E)$. (The unlabeled morphisms are the natural degree 1 maps.)

13.1. Definition of micro-support. Let $\mathfrak{I r r}\left(L_{P}\right)$ denote the irreducible algebraic representations of $L_{P}$. Recall we have decomposed $L_{P}=M_{P} A_{P}$, where $A_{P}$ is the identity component of the maximal $\mathbb{Q}$-split torus in the center of $L_{P}$. For any $V \in \mathfrak{I} \mathfrak{r r}\left(L_{P}\right)$, let $\xi_{V}$ be the character by which $A_{P}$ acts on $V$ and let $\mathbb{C}_{\xi_{V}}$ be the corresponding 1-dimensional representation. We can then write $V$ as $\left.V\right|_{M_{P}} \otimes \mathbb{C}_{\xi_{V}}$. Recall that $\Delta_{P}$ denotes the simple $A_{P}$-weights of the adjoint action of (a lift of) $L_{P}$ on $\mathfrak{n}_{P}=\operatorname{Lie}\left(N_{P}\right)$, and that the parabolic $\mathbb{Q}$-subgroups $Q \geq P$ are parametrized by subsets $\Delta_{P}^{Q}$ of $\Delta_{P}$. Consequently we can define parabolic $\mathbb{Q}$-subgroups $P \leq Q_{V} \leq Q_{V}^{\prime}$ by setting

$$
\begin{aligned}
& \Delta_{P}^{Q_{V}}=\left\{\alpha \in \Delta_{P} \mid\left(\xi_{V}+\rho, \alpha\right)<0\right\}, \\
& \Delta_{P}^{Q_{V}^{\prime}}=\left\{\alpha \in \Delta_{P} \mid\left(\xi_{V}+\rho, \alpha\right) \leq 0\right\} ;
\end{aligned}
$$

here $\rho$ is one-half the sum of the $A_{P}$-weights of $\mathfrak{n}_{P}$ (counted with multiplicity).

Let $\mathcal{M}$ be an $\mathcal{L}$-module on $\widehat{X}$. Define $\operatorname{SS}(\mathcal{M})$ to consist of those $V \in \mathfrak{I r r}\left(L_{P}\right)$ (for $P \in \mathcal{P}$ ) such that

$$
\begin{aligned}
& \left(\left.V\right|_{M_{P}}\right)^{*} \cong \overline{\left.V\right|_{M_{P}}}, \text { and } \\
& H\left(i_{P}^{*} \hat{\imath}_{Q}^{!} \mathcal{M}\right)_{V} \neq 0 \text { for some } Q_{V} \leq Q \leq Q_{V}^{\prime} .
\end{aligned}
$$

Here $H\left(i_{P}^{*} \hat{\imath}_{Q}^{!} \mathcal{M}\right)_{V}$ denotes the $V$-isotypical component of $H\left(i_{P}^{*} \hat{\imath}_{Q}^{!} \mathcal{M}\right)$. Let

$$
c(V ; \mathcal{M}) \leq d(V ; \mathcal{M})
$$

be the least and greatest degrees for which (ii) holds. 
Condition (i) says that $\left.V\right|_{M_{P}}$ is conjugate self-contragredient. This condition is automatic if $D_{P}$ is equal-rank, $\mathbb{C}$-rank $M_{P}=\operatorname{rank} K_{P}$ (see the discussion later in $§ 13.2$ ). Condition (ii) may be interpreted geometrically as follows. Consider the long exact sequence of the pair $\left(U, U \backslash\left(U \cap \widehat{X}_{Q}\right)\right)$ where $U$ is a small neighborhood of a point of $X_{P}$. Since $H\left(i_{P}^{*} \hat{\imath}_{Q}^{!} \mathcal{M}\right)_{V} \cong H\left(U, U \backslash\left(U \cap \widehat{X}_{Q}\right) ; \mathcal{M}\right)_{V}$, equation (ii) is equivalent to

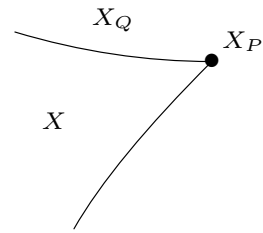

$$
H(U ; \mathcal{M})_{V} \quad \longrightarrow \quad H\left(U \backslash\left(U \cap \widehat{X}_{Q}\right) ; \mathcal{M}\right)_{V}
$$

failing to be an isomorphism in some degree. Note that a functor like $i_{P}^{*} \hat{\imath}_{Q}^{!}$was used by Goresky and MacPherson [35] to describe the local contributions to a Lefschetz fixed point formula; more recently it has been used in Braden's work [19] on hyperbolic localization.

We say that an element $V \in \mathrm{SS}(\mathcal{M})$ belongs to $\operatorname{SS}_{\text {ess }}(\mathcal{M})$, the essential microsupport, if furthermore

$$
H\left(i_{P}^{*} \hat{\imath}_{Q_{V}}^{\prime} \mathcal{M}\right)_{V} \longrightarrow H\left(i_{P}^{*} \hat{\imath}_{Q_{V}^{\prime}}^{\prime} \mathcal{M}\right)_{V} \quad \text { is nonzero. }
$$

All of the above was for an $\mathcal{L}$-module on $\widehat{X}$. Consider more generally an admissible subspace $W \subseteq \widehat{X}$ which has a unique maximal stratum $X_{R}$. We define the micro-support and the essential micro-support of an $\mathcal{L}$-module on $W$ similarly except that we restrict $V$ to belong to $\coprod_{P \in \mathcal{P}(W)} \mathfrak{I r r}\left(L_{P}\right)$ and replace $Q_{V}$ and $Q_{V}^{\prime}$ by parabolic $\mathbb{Q}$-subgroups $Q_{V}^{R}$ and $Q_{V}^{\prime R}$ defined by

$$
\begin{aligned}
& \Delta_{P}^{Q_{V}^{R}}=\left\{\alpha \in \Delta_{P}^{R} \mid\left(\xi_{V}+\rho, \alpha\right)<0\right\}, \\
& \Delta_{P}^{Q_{V}^{\prime R}}=\left\{\alpha \in \Delta_{P}^{R} \mid\left(\xi_{V}+\rho, \alpha\right) \leq 0\right\} .
\end{aligned}
$$

13.2. Example: Micro-support of $\boldsymbol{i}_{\boldsymbol{G}} \boldsymbol{E}$. We illustrate the definition by calculating the micro-support of $i_{G *} E[\mathbf{6 2}]$. This also gives us an opportunity to introduce Kostant's theorem which will be important later.

For $\mathcal{M}=i_{G *} E$, we have $E_{P}=0$ for all $P \neq G$ and thus $\hat{\imath}_{Q} \mathcal{M}=0$ unless $Q=G$; if $Q=G$ we have $\hat{\imath}_{G}^{!} \mathcal{M}=\mathcal{M}$. Thus for any $P \in \mathcal{P}$, we only need to consider irreducible $L_{P}$-modules $V$ such that $Q_{V}^{\prime}=G$, that is, such that

$$
\left(\xi_{V}+\rho, \alpha\right) \leq 0 \quad \text { for all } \alpha \in \Delta_{P}
$$

such a $V$ will actually be in $\operatorname{SS}\left(i_{G *} E\right)$ if $\left(\left.V\right|_{M_{P}}\right)^{*} \cong \overline{\left.V\right|_{M_{P}}}$ and if $V$ occurs in $H\left(i_{P}^{*} i_{G *} E\right)=H\left(\mathfrak{n}_{P} ; E\right)$.

We therefore need to know the irreducible components of $H\left(\mathfrak{n}_{P} ; E\right)$; in fact a theorem of Kostant [45] completely describes $H\left(\mathfrak{n}_{P} ; E\right)$ as an $L_{P}$-module. Since this will be essential later as well, we review it here. First some notation. Let $\mathfrak{h}=\mathfrak{h}_{M_{P}}+\mathfrak{a}_{P}$ be a Cartan subalgebra of $\mathfrak{l}_{P}$. Via a lift we may view $\mathfrak{h}$ as a Cartan subalgebra of $\mathfrak{g}$ as well and we choose a set of simple $\mathbb{C}$-roots ${ }_{\mathbb{C}} \Delta$ for $\mathfrak{g}_{\mathbb{C}}$ such that the roots in $\mathfrak{n}_{P \mathbb{C}}$ are positive; as usual, let $\rho$ denote one-half the sum of the positive 
roots (its restriction to $\mathfrak{a}_{P}$ agrees with the previous definition of $\rho$ ). Let $W$ be the Weyl group of $\mathfrak{g}_{\mathbb{C}}$ and let $W^{P}$ denote the subgroup corresponding to the Weyl group of $\mathfrak{l}_{P \mathbb{C}}$. The length of $w \in W$ is denoted $\ell(w)$ and we let $W_{P}$ be the set of minimal length coset representatives of $W^{P} \backslash W$. Assume $E$ is irreducible with highest weight $\lambda$. Kostant's theorem says that

$$
H\left(\mathfrak{n}_{P} ; E\right)=\bigoplus_{w \in W_{P}} V_{w(\lambda+\rho)-\rho}[-\ell(w)],
$$

where $V_{\mu}$ denotes the irreducible $L_{P}$-module with highest weight $\mu$.

We also need to understand when $\left.V\right|_{M_{P}}$ is conjugate self-contragredient. The involution $\left.V\right|_{M_{P}} \mapsto\left(\overline{\left.V\right|_{M_{P}}}\right) *$ on representations induces an involution on highest weights, $\left.\mu\right|_{h_{M_{P}}} \mapsto \tau_{P}\left(\left.\mu\right|_{h_{M_{P}}}\right)$. It is always the case that $\tau_{P}\left(\left.\rho\right|_{\mathfrak{h}_{M_{P}}}\right)=\left.\rho\right|_{\mathfrak{h}_{M_{P}}}$, so $\left.V_{w(\lambda+\rho)-\rho}\right|_{M_{P}}$ is conjugate self-contragredient if and only if

$$
\tau_{P}\left(\left.w(\lambda+\rho)\right|_{\mathfrak{h}_{M_{P}}}\right)=\left.w(\lambda+\rho)\right|_{\mathfrak{h}_{M_{P}}} .
$$

The involution $\tau_{P}$ is described in [13]; it is the composition of the opposition involution and the "*-action" of complex conjugation [75]. Concretely, let ${ }_{\mathbb{C}} \Delta^{P} \subseteq$ ${ }_{\mathbb{C}} \Delta$ denote the simple $\mathbb{C}$-roots of $\mathfrak{l}_{P \mathbb{C}}$. Then

$$
\tau_{P}=\left(-w_{0}^{P}\right) \circ\left(w_{c}^{P} c\right)
$$

where $w_{0}^{P} \in W^{P}$ is the longest element, $c$ is complex conjugation, and $w_{c}^{P} \in W^{P}$ is such that $w_{c}^{P}\left(c_{\mathbb{C}} \Delta^{P}\right)={ }_{\mathrm{C}} \Delta^{P}$. Even more concretely, if $\mathfrak{h}$ is a fundamental (that is, maximally compact) $\theta$-stable Cartan subalgebra of $\mathfrak{l}_{P}$ and the positive system is chosen so that ${ }_{\mathbb{C}} \Delta^{P}$ is $\theta$-stable, then $\tau_{P}=\theta$.

The micro-support of $i_{G *} E$ is thus

$$
\begin{aligned}
\mathrm{SS}\left(i_{G *} E\right)=\coprod_{P}\left\{V_{w(\lambda+\rho)-\rho} \mid\right. & (w(\lambda+\rho), \alpha) \leq 0 \text { for all } \alpha \in \Delta_{P}, \\
& \left.\tau_{P}\left(\left.w(\lambda+\rho)\right|_{\mathfrak{h}_{M_{P}}}\right)=\left.w(\lambda+\rho)\right|_{\mathfrak{h}_{M_{P}}}\right\} ;
\end{aligned}
$$

to obtain an expression for the essential micro-support $\operatorname{SS}_{\text {ess }}\left(i_{G *} E\right)$, we impose instead the strict inequality $(w(\lambda+\rho), \alpha)<0$. For $V=V_{w(\lambda+\rho)-\rho} \in \operatorname{SS}\left(i_{G *} E\right)$, we have

$$
c\left(V ; i_{G *} E\right)=d\left(V ; i_{G *} E\right)=\ell(w) .
$$

\section{A vanishing theorem for the cohomology of an $\mathcal{L}$-module}

The justification for the definition of $\operatorname{SS}(\mathcal{M})$ is that it enters into a general vanishing theorem for the cohomology of an $\mathcal{L}$-module (see Theorem 14.1 below).

First some definitions. Let $\mathcal{M}$ be an $\mathcal{L}$-module on $\widehat{X}$ and let $V \in \operatorname{SS}(\mathcal{M})$ be an $L_{P}$-module. Let $\mu$ be the highest weight of $V$ with respect to a fundamental Cartan subalgebra $\mathfrak{h}$ of $\mathfrak{l}_{P}$ and a $\theta$-stable positive system; view $\mu$ as an element of $\mathfrak{l}_{P \mathbb{C}}^{*}$ by extending it to be 0 on all root spaces. Let $L_{P}(\mu) \subseteq L_{P}$ be the stabilizer of $\mu$ for the coadjoint action; this is a reductive $\mathbb{R}$-subgroup whose $\mathbb{C}$-roots are those $\mathbb{C}$-roots of $L_{P}$ which are orthogonal to $\mu$. Let

$$
D_{P}(V)=L_{P}(\mu) /\left(K_{P} \cap L_{P}(\mu)\right) A_{P}
$$

be the associated symmetric space. The space $D_{P}(V)$ is not well-defined, even up to isomorphism, since it depends on the choice of a $\theta$-stable positive system; we will assume however that the positive system has been chosen so that $D_{P}(V)$ has the maximum possible dimension. 
Define

$$
\begin{aligned}
& c(\mathcal{M})=\inf _{V \in \operatorname{SS}(\mathcal{M})} \frac{1}{2}\left(\operatorname{dim} D_{P}-\operatorname{dim} D_{P}(V)\right)+c(V ; \mathcal{M}), \\
& d(\mathcal{M})=\sup _{V \in \operatorname{SS}(\mathcal{M})} \frac{1}{2}\left(\operatorname{dim} D_{P}+\operatorname{dim} D_{P}(V)\right)+d(V ; \mathcal{M}) .
\end{aligned}
$$

As we will see in Theorem 16.1, the significance of $\left(\operatorname{dim} D_{P} \pm \operatorname{dim} D_{P}(V)\right) / 2$ is that this is the range of degrees in which $H_{(2)}\left(X_{P} ; \mathbb{V}\right)$ can be nonzero. In these definitions, one can also consider just $V \in \mathrm{SS}_{\text {ess }}(\mathcal{M})$ and redefine $c(V ; \mathcal{M}) \leq d(V ; \mathcal{M})$ to be the least and greatest degrees in which (ii)' is nonzero; one can prove that the same values of $c(\mathcal{M})$ and $d(\mathcal{M})$ are obtained.

The following vanishing theorem is proved in [61, Theorem 10.4]:

TheOrem 14.1. Let $\mathcal{M}$ be an $\mathcal{L}$-module on $\widehat{X}$. Then

$$
H^{j}(\widehat{X} ; \mathcal{M})=0 \quad \text { for } j \notin[c(\mathcal{M}), d(\mathcal{M})] .
$$

In particular, if $\mathrm{SS}(\mathcal{M})=\emptyset$ then $H(\widehat{X} ; \mathcal{M})=0$ identically.

We will sketch a proof of the theorem in $\S 17$. It has a number of ingredients, including the author's past work on tilings of locally symmetric spaces [60], various analytic estimates, and a vanishing theorem for $L^{2}$-cohomology which we will recall in $\S 16$.

\section{Application: a vanishing theorem for ordinary cohomology}

One can immediately apply Theorem 14.1 and our calculation of $\operatorname{SS}_{\text {ess }}\left(i_{G *} E\right)$ to obtain a vanishing theorem for the ordinary cohomology $H(X ; \mathbb{E})$. This applies to all $X$ and $E$, however in special cases we can be more explicit regarding the degree bounds. For example, we have proved [62, Theorem 5]:

THEOREM 15.1. If $D$ is equal-rank and $E$ has regular highest weight, then

$$
H^{j}(X ; E)=0 \quad \text { for } j<\frac{1}{2} \operatorname{dim} X .
$$

The same theorem has been announced by Li and Schwermer [48] in a slightly strengthened form. They drop the equal-rank condition and replace the vanishing range by

$$
j<\frac{1}{2}\left(\operatorname{dim} X-\left(\mathbb{C}-\operatorname{rank}^{0} G-\operatorname{rank} K\right)\right) ;
$$

it is clear that this strengthened form also follows from our proof in [62]. Theorem 15.1 answers a question of Tilouine $[\mathbf{5 3}, \S 8.7]$ in the Hermitian case; the case $G=R_{k / \mathbb{Q}} \operatorname{GSp}_{4}(\mathbb{R})$ where $k$ is a totally real number field was proved in [74] using results of Franke [29]. For an application of the theorem see Mauger's thesis [51].

In order to deduce Theorem 15.1 from Theorem 14.1, equation (43) shows that we need an estimate on $\ell(w)$. The following basic lemma provides the needed estimate; it will also be crucial later in studying the micro-support of $\mathcal{I}_{p} \mathcal{C}(\widehat{X} ; E)$ and its behavior under functorial operations, Theorems 18.1 and 19.1.

Lemma 15.2. Let $E$ be an irreducible $G$-module with highest weight $\lambda$. Let $P$ be a parabolic $\mathbb{Q}$-subgroup and let $w \in W_{P}$. Set $V=V_{w(\lambda+\rho)-\rho}$ and assume that $\left.V\right|_{M_{P}}$ conjugate self-contragredient.

(i) If $(w(\lambda+\rho), \alpha) \leq 0$ for all $\alpha \in \Delta_{P}$, then

$$
\ell(w) \geq \frac{1}{2}\left(\operatorname{dim} \mathfrak{n}_{P}+\operatorname{dim} \mathfrak{n}_{P}(V)\right) .
$$


(ii) If $(w(\lambda+\rho), \alpha) \geq 0$ for all $\alpha \in \Delta_{P}$, then

$$
\ell(w) \leq \frac{1}{2}\left(\operatorname{dim} \mathfrak{n}_{P}-\operatorname{dim} \mathfrak{n}_{P}(V)\right) .
$$

The quantity $\mathfrak{n}_{P}(V)$ in the lemma is defined as follows; see $[\mathbf{6 1}, \S 24.1]$ for more details. Let $\mu$ be the highest weight of $V$ for a fundamental $\theta$-stable Cartan subalgebra of $\mathfrak{l}_{P}$ and $\theta$-stable positive system. Let $\mathfrak{n}_{P}(\mu)$ be the sum of the irreducible $L_{P}(\mu)$-submodules of $\mathfrak{n}_{P \mathbb{C}}$ whose weights are stable under the action of $-\tau_{P}$; observe that $-\tau_{P}$ is complex conjugation in this situation so that $\mathfrak{n}_{P}(\mu)$ contains in particular all positive real root spaces. Let $\mathfrak{n}_{P}(V)$ be any of the $\mathfrak{n}_{P}(\mu)$ with maximal dimension as we vary the $\theta$-stable positive system.

We can now deduce the theorem (in its strengthened form). The KostantSugiura classification of Cartan subalgebras [44], [72], [73] shows that rank $K-$ $\operatorname{rank} K_{P}$ is at most the number of positive real roots. Thus

$$
\operatorname{dim} \mathfrak{n}_{P}(V) \geq \operatorname{rank} K-\operatorname{rank} K_{P} .
$$

The hypothesis that $E$ has regular highest weight implies that $\operatorname{dim} D_{P}(V)=$ $\mathbb{C}$-rank $M_{P}-\operatorname{rank} K_{P}$ for any $V \in \mathrm{SS}\left(i_{G *} E\right)$. These facts and Lemma 15.2(i) allow one to estimate

$$
\begin{aligned}
& \frac{1}{2}\left(\operatorname{dim} D_{P}-\operatorname{dim} D_{P}(V)\right)+c\left(V ; i_{G *} E\right) \\
& \quad \geq \frac{1}{2}\left(\operatorname{dim} D_{P}+\operatorname{dim} \mathfrak{n}_{P}-\left(\mathbb{C}-\operatorname{rank} M_{P}-\operatorname{rank} K\right)\right) \\
& \quad=\frac{1}{2}\left(\operatorname{dim} X-\left(\mathbb{C}-\operatorname{rank}^{0} G-\operatorname{rank} K\right)\right) .
\end{aligned}
$$

Sketch OF THE PROOF OF Lemma 15.2. We will sketch the argument with $\operatorname{dim} \mathfrak{n}_{P}(V)$ replaced by $\operatorname{rank} K-\operatorname{rank} K_{P}$; see [61, Lemma 24.2] for the complete proof. Recall that $\ell(w)$ can be viewed as the number of positive $\mathbb{C}$-roots $\gamma$ for which $w^{-1} \gamma$ is negative; for $w \in W_{P}$ all these roots are in $\mathfrak{n}_{P \mathbb{C}}$. Since $\lambda+\rho$ is strictly dominant, $(w(\lambda+\rho), \gamma)=\left(\lambda+\rho, w^{-1} \gamma\right)$ is never zero and will be negative precisely when $w^{-1} \gamma<0$. Thus $\ell(w)$ is the number of roots $\gamma$ in $\mathfrak{n}_{P \mathbb{C}}$ for which

$$
(w(\lambda+\rho), \gamma) \leq 0 .
$$

However complex conjugation defines an involution $\gamma \mapsto \bar{\gamma}$ on the roots in $\mathfrak{n}_{P \mathbb{C}}$; we will prove (i) by showing that (45) holds for at least one of $\gamma$ and $\bar{\gamma}$.

Assume that $\mathfrak{h}=\mathfrak{h}_{M_{P}}+\mathfrak{a}_{P}$ is a fundamental $\theta$-stable Cartan subalgebra of $\mathfrak{l}_{P}$ and that the set of positive roots of $\mathfrak{l}_{P \mathbb{C}}$ are $\theta$-stable. Decompose $\mathfrak{h}=\mathfrak{h}_{M_{P}, 1}+$ $\left(\mathfrak{h}_{M_{P},-1}+\mathfrak{a}_{P}\right)$ according to the \pm 1 -eigenspaces of the Cartan involution. In this case, the operator $\tau_{P}$ is simply $\theta[\mathbf{1 3}]$, so the condition that $\left(\left.V_{w(\lambda+\rho)-\rho}\right|_{M_{P}}\right)^{*} \cong$ $\overline{\left.V_{w(\lambda+\rho)-\rho}\right|_{M_{P}}}$ is equivalent to $\left.w(\lambda+\rho)\right|_{\mathfrak{h}_{M_{P},-1}}=0$. It follows that

$$
(w(\lambda+\rho), \gamma)=\left(w(\lambda+\rho),\left.\gamma\right|_{\mathfrak{h}_{M_{P}, 1}}\right)+\left(w(\lambda+\rho),\left.\gamma\right|_{\mathfrak{a}_{P}}\right) .
$$

Since $\left.\gamma\right|_{\mathfrak{a}_{P}}$ is a linear combination with nonnegative coefficients of $\alpha \in \Delta_{P}$, the second term is nonpositive by hypothesis; on the other hand, the first term is negated when $\gamma$ is replaced by $\bar{\gamma}$. Thus (45) holds for one of either $\gamma$ or $\bar{\gamma}$ as desired. In particular it holds for all roots such that $\gamma=\bar{\gamma}$, that is, the positive real roots. Since, as we have already noted, the number of positive real roots is at least $\operatorname{rank} K-\operatorname{rank} K_{P}$, assertion (i) follows with $\operatorname{dim} \mathfrak{n}_{P}(V)$ substituted by $\operatorname{rank} K-\operatorname{rank} K_{P}$. Assertion (ii) is proven similarly. 


\section{A vanishing theorem for $L^{2}$-cohomology}

As we will see in the next section, the proof of Theorem 14.1 will be reduced to the study of the $L^{2}$-cohomology groups $H_{(2)}\left(X_{P} ; \mathbb{V}\right)$ for all $P \in \mathcal{P}$ and all $L_{P^{-}}$ modules $V \in \mathrm{SS}(\mathcal{M})$. Thus we need a vanishing theorem for $L^{2}$-cohomology. The theorem below was proved by the author and Stern in [65] though it is not stated explicitly in this form; it is based on work of Raghunathan [56], [57]. The statement and proof appeared in [61, Theorem 14.4].

THEOREM 16.1. Let $X$ be a locally symmetric space and let $E$ be a representation of $G$ with corresponding metrized locally constant sheaf $\mathbb{E}$.

(i) If $\left(\left.E\right|_{0_{G}}\right)^{*} \approx \overline{\left.E\right|_{{ }^{\circ} G}}$, then $H_{(2)}(X ; \mathbb{E})=0$.

(ii) If $\left(\left.E\right|_{{ }^{\circ} G}\right)^{*} \cong \overline{E \mid{ } G}$, then $H_{(2)}^{j}(X ; \mathbb{E})=0$ for

$$
\begin{aligned}
& \quad j \notin\left[\frac{1}{2}(\operatorname{dim} D-\operatorname{dim} D(E)), \frac{1}{2}(\operatorname{dim} D+\operatorname{dim} D(E))\right] \\
& \text { and } H_{(2)}^{(\operatorname{dim} D-\operatorname{dim} D(E)) / 2}(X ; \mathbb{E}) \text { is Hausdorff. }
\end{aligned}
$$

The proof of the theorem is based on the following criterion which will be used in the proof of Theorem 14.1 later as well:

Proposition 16.2. Let $M$ be a complete Riemannian manifold with a metrized locally constant sheaf $\mathbb{E}$. Then for every $j$, the following two conditions are equivalent.

(i) $H_{(2)}^{j}(M ; \mathbb{E})=0$ and $H_{(2)}^{j+1}(M ; \mathbb{E})$ is Hausdorff;

(ii) there exists $c>0$ such that

$$
\|d \phi\|^{2}+\left\|d^{*} \phi\right\|^{2} \geq c\|\phi\|^{2}
$$

for all compactly supported smooth forms $\phi$ of degree $j$.

The complex of compactly supported forms in $A_{(2)}(X ; \mathbb{E})$ may be identified as in [18, VII.2.7], [50] with a Koszul complex $C\left({ }^{0} \mathfrak{g}, K ; A_{c}^{0}\left(\Gamma \backslash^{0} G\right) \otimes E\right)$. Thus the differential may be decomposed $d=d_{0}+d_{1}$, where $d_{0}$ corresponds to the algebraic action of ${ }^{0} \mathfrak{g}$ on $E$ and $d_{1}$ corresponds to the action of ${ }^{0} \mathfrak{g}$ on $A_{c}^{0}\left(\Gamma \backslash^{0} G\right)$ by differentiation. One calculates using integration by parts that

$$
\|d \phi\|^{2}+\left\|d^{*} \phi\right\|^{2} \geq\left(\Delta_{0} \phi, \phi\right)
$$

where $\Delta_{0}$ is the nonnegative algebraic Laplacian corresponding to $d_{0}$; it is the Laplacian for the complex of invariant forms $C\left({ }^{0} \mathfrak{g}, K ; E\right)$. A careful analysis of the possible zero eigenvectors of $\Delta_{0}$ yields Theorem 16.1 .

Alternatively, note that a vanishing theorem for $(\mathfrak{g}, K)$-cohomology of unitary representations was proved by Vogan and Zuckerman [77] based on work by Kumaresan [46]; this implies a vanishing theorem for $L^{2}$-cohomology (at least the Hausdorff part) which one can show [63] is equivalent with Theorem 16.1.

\section{Proof of the vanishing theorem for the cohomology of an $\mathcal{L}$-module}

In the following pages we sketch a proof of Theorem 14.1, the vanishing theorem for the cohomology $H(\widehat{X} ; \mathcal{M})$ of an $\mathcal{L}$-module $\mathcal{M}=(E ., f .$.$) . Most steps of the proof$ are accompanied by an illustrative figure. 


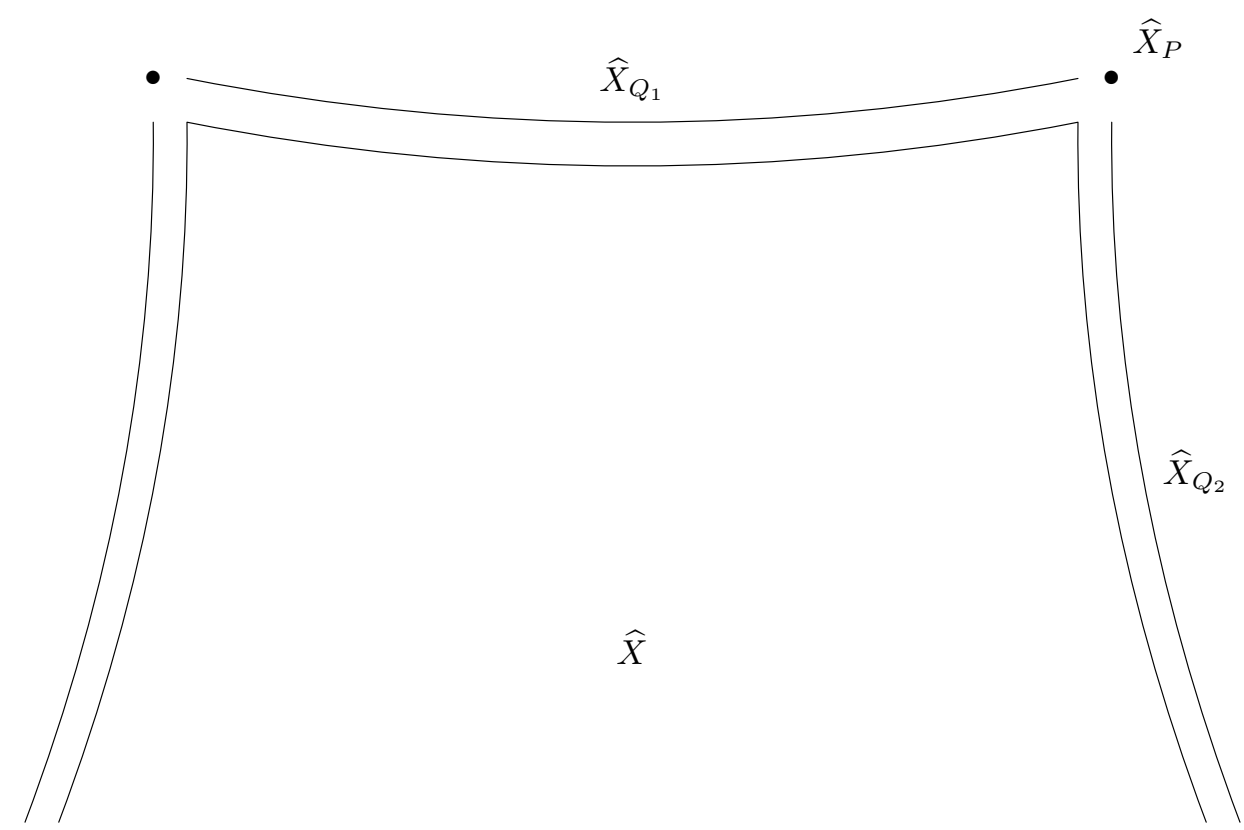

Figure 16. The complex $\Gamma(\widehat{X}, \mathcal{S}(\mathcal{M}))$ lives on the disjoint union $\coprod_{P} \widehat{X}_{P}$

The cohomology $H(\widehat{X} ; \mathcal{M})$ may be computed as the cohomology of the complex of global sections of the fine realization sheaf $\mathcal{S}(\mathcal{M})$ defined in $\$ 12.4$. Ignoring the differentials, this complex is

$$
\Gamma(\widehat{X}, \mathcal{S}(\mathcal{M}))=\bigoplus_{P \in \mathcal{P}} A_{\mathrm{sp}}\left(\widehat{X} ; \mathbb{E}_{P}\right)
$$

Thus a "form" $\phi$ is really a collection $\left(\phi_{P}\right)_{P \in \mathcal{P}}$ of special differential forms on the various strata as pictured in Figure 16. The differential is

$$
d \phi=d\left(\phi_{P}\right)_{P \in \mathcal{P}}=\left(\sum_{P \leq Q} d_{P Q} \phi_{Q}\right)_{P \in \mathcal{P}}=\left(d_{P} \phi_{P}+\sum_{P \leq Q} f_{P Q}\left(\left.\phi_{Q}\right|_{\widehat{X}_{P}}\right)\right)_{P \in \mathcal{P}}
$$

where $d_{P}$ denotes the de Rham differential and we write $\left.\phi_{Q}\right|_{\widehat{X}_{P}}$ for $k_{P Q}\left(\phi_{Q}\right)$. 


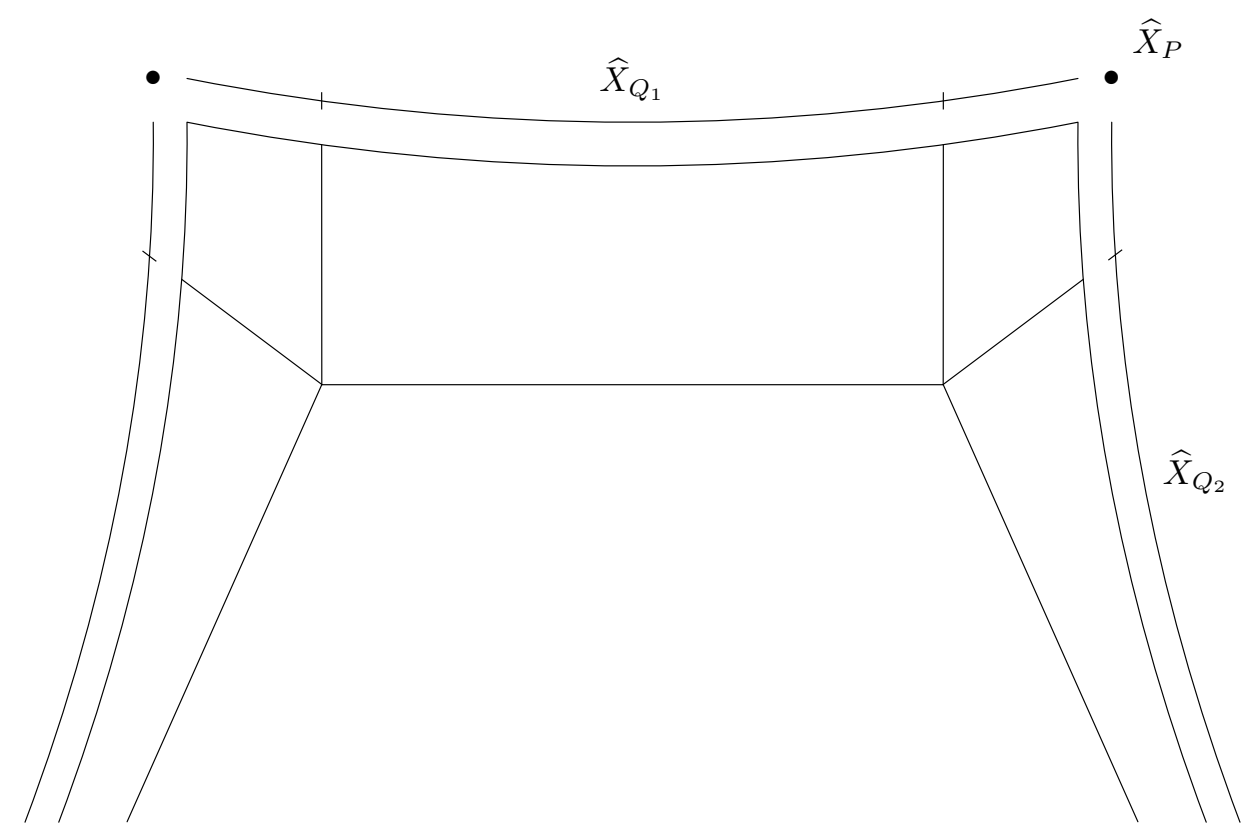

Figure 17. The Arthur-Langlands Partition

We would like to use Hodge theory to calculate the cohomology of this complex and for that we need to consider the $L^{2}$-norm

$$
\|\phi\|^{2} \equiv \sum_{P}\left\|\phi_{P}\right\|^{2} .
$$

However in the locally symmetric metric on $X_{P}$, the boundary is at infinite distance. Thus the condition $\|\phi\|<\infty$ imposes nontrivial $L^{2}$-growth conditions on the $\phi_{P}$ which would change the cohomology.

The solution is to replace each $X_{P}$ with a diffeomorphic copy which is embedded as a domain in $X_{P}$ with compact closure. If the diffeomorphic embedding extended to the manifold with corners $\bar{X}_{P}$ the new boundary faces would now be at finite distance. To do this naturally, consider the Arthur-Langlands partition [1] of $X$. This was extended by the author to a partition of $\bar{X}$ (and hence $\widehat{X}$ ) in [60]; see Figure 17. 


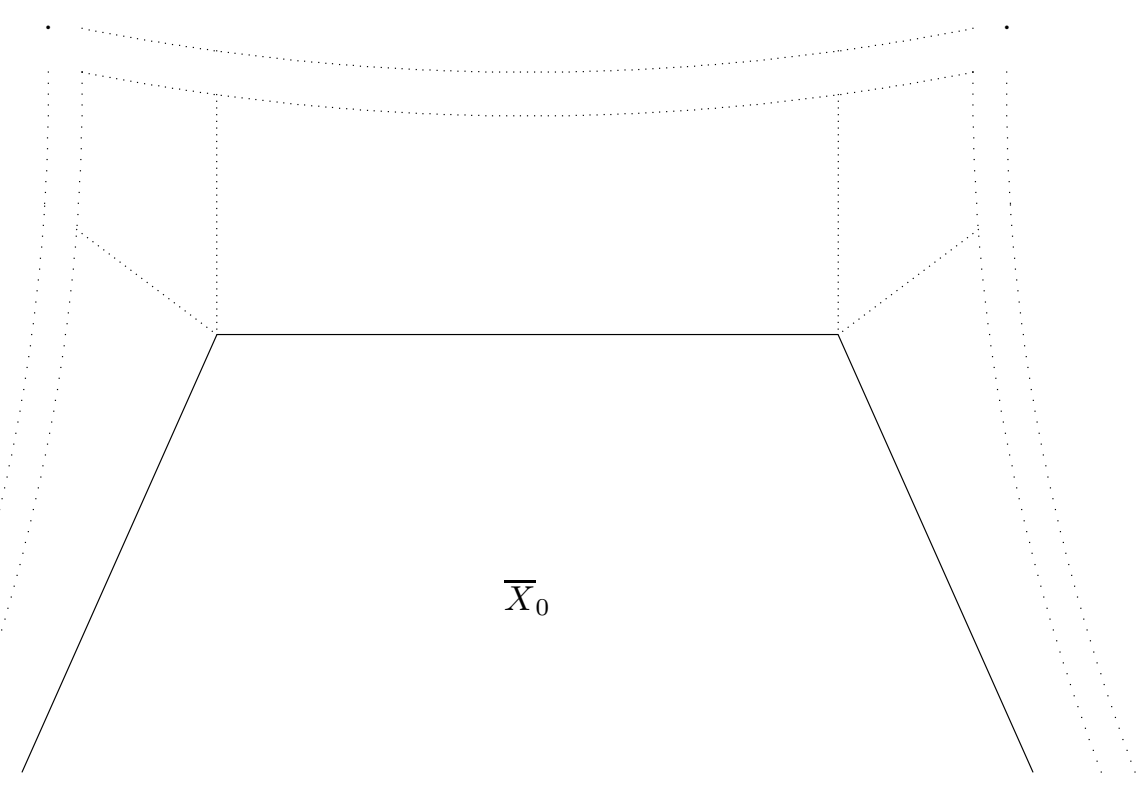

Figure 18. The Central Region

The Arthur-Langlands partition divides $X$ into disjoint regions indexed by $\mathcal{P}$; it depends on a certain parameter $\mathbf{b}=\left(b_{P}\right)_{P \in \mathcal{P}}$, where $b_{P} \in \mathcal{A}_{P}^{G}$. We will only need here the central region $\bar{X}_{0}$, which is the one indexed by $G \in \mathcal{P}$. Recall that although the decomposition $D=\mathcal{A}_{P}^{G} \times e_{P}$ does not descend to a decomposition $\mathcal{A}_{P}^{G} \times Y_{P}$ of $X$, such a decomposition does hold within an appropriate cylindrical set $W_{P}$ (see (15) in $\S 6.3$ ). We can use this decomposition to describe $\bar{X}_{0}$ :

$$
\bar{X}_{0} \cap W_{P}=\left(\left({ }^{-} A_{P}^{G} \cdot b_{P}\right) \times Y_{P}\right) \cap W_{P} .
$$

In $[60]$ it was shown that there exists a natural piecewise analytic diffeomorphism (depending on another parameter) of $\bar{X}$ with $\bar{X}_{0}$. So the next step is to throw away everything outside of $\bar{X}_{0}$ and start over.

We endow the central region $\bar{X}_{0}$ with the metric induced by restriction from the locally symmetric metric on $X$. Since $\bar{X}_{0}$ is then a compact Riemannian manifold with corners, a smooth form on $\bar{X}_{0}$ or any of its boundary faces will automatically have finite $L^{2}$-norm. 


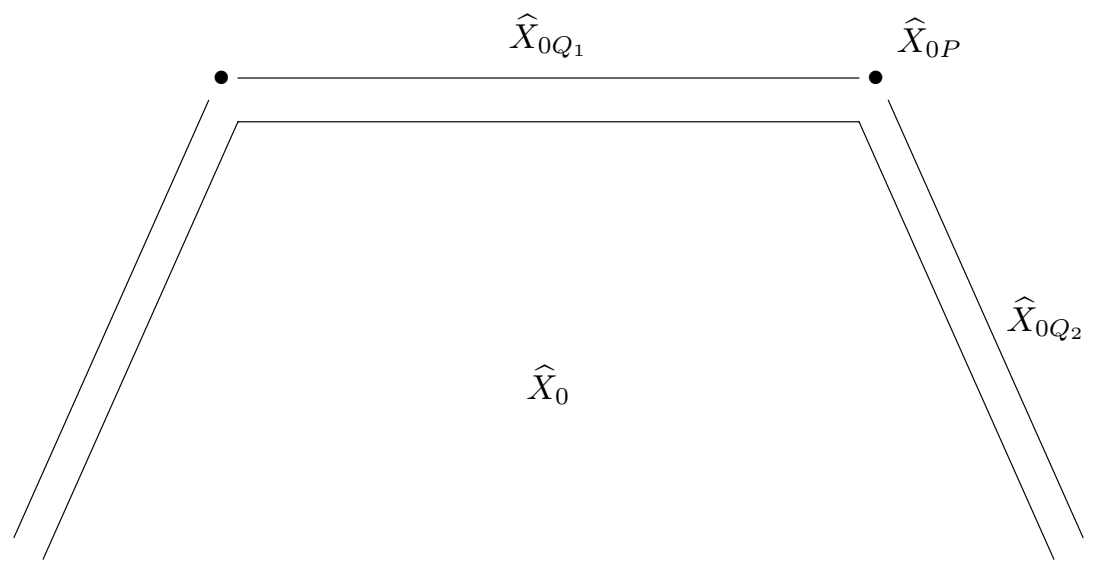

Figure 19. The complex $\Gamma\left(\widehat{X}_{0}, \mathcal{S}_{0}(\mathcal{M})\right)$ lives on the disjoint union $\coprod_{P} \widehat{X}_{0 P}$

Since $\bar{X}_{0}$ is diffeomorphic with $\bar{X}$ we can mimic the construction of $\widehat{X}$ and $\mathcal{S}(\mathcal{M})$ to obtain a realization $\mathcal{S}_{0}(\mathcal{M})$ on $\widehat{X}_{0}$ whose global sections also compute $H(\widehat{X} ; \mathcal{M})$; see Figure 19 . Since we have removed the difficulty with $L^{2}$-growth conditions, Hodge theory shows that classes in $H(\widehat{X} ; \mathcal{M})$ have harmonic representatives in $\Gamma\left(\widehat{X}_{0}, \mathcal{S}_{0}(\mathcal{M})\right)$. This is the analogue of the classical Hodge theorem for a compact Riemannian manifold.

We now follow an argument from [65] however there is an added combinatorial complication since our "forms" are actually collections of forms and there are interaction terms in the differential. The aim is to show that there exists $c>0$ such that the estimate

$$
\|d \phi\|^{2}+\left\|d^{*} \phi\right\|^{2} \geq c\|\phi\|^{2}
$$

holds for all $\phi$ with degree $j \notin[c(\mathcal{M}), d(\mathcal{M})]$. This will suffice to prove the theorem by (a generalization of) Proposition 16.2. 


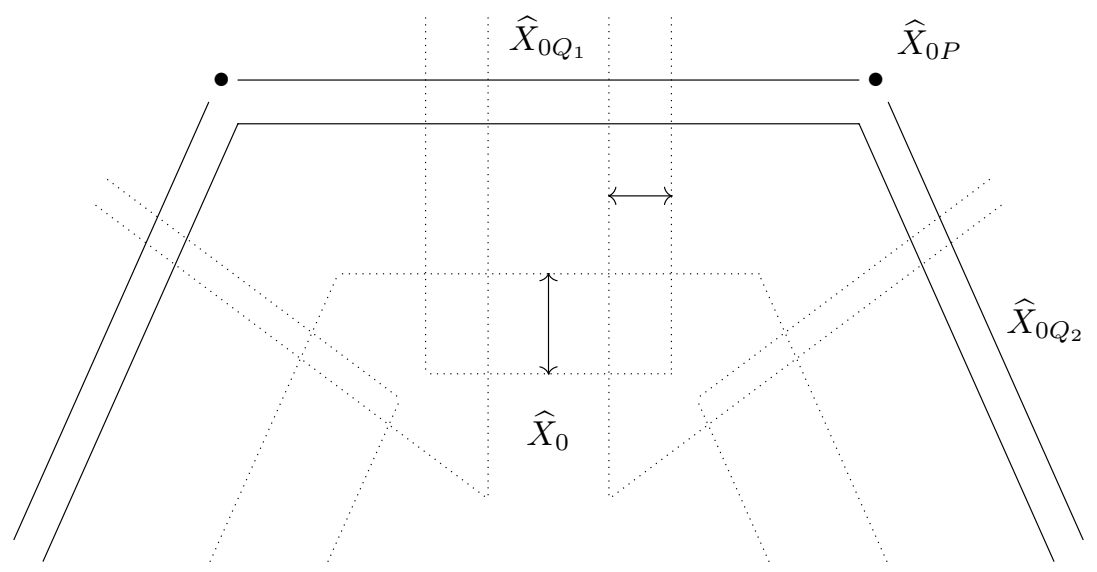

Figure 20. Covering by cylindrical sets $W_{P}$

Let $\left\{W_{P}\right\}_{P \in \mathcal{P}}$ be a cylindrical cover of $\bar{X}$ as in (16) of $\S 6.3$; we can arrange that for all $P \in \mathcal{P}$, the cylindrical set $W_{P}=\left(\bar{A}_{P}^{G+} \cdot s_{P}\right) \times O_{P}$ projects to a relatively compact subset of $X_{0 P}$. Such a cover induces a cover $\left\{W_{P}\right\}_{P \leq Q}$ of each $\widehat{X}_{0 Q}$; for simplicity we denote the elements of such a cover again by $W_{P}$. The situation is pictured in Figure 20.

Assume that for each $P \in \mathcal{P}$ we can establish an estimate

$$
\|d \phi\|^{2}+\left\|d^{*} \phi\right\|^{2} \geq c_{P}\|\phi\|^{2}
$$

for all $\phi$ with compact support in $\widehat{X}_{0} \cap W_{P}$ and degree $j \notin[c(\mathcal{M}), d(\mathcal{M})]$. Here $c_{P}>0$ is to be a constant independent of $O_{P}$. If this is possible, and if there exists a partition of unity $\left\{\eta_{P}\right\}_{P \in \mathcal{P}}$ with $\left|d \eta_{P}\right|<\epsilon$ for all $P$ (where $\epsilon>0$ depends on $\left.\left\{c_{P}\right\}_{P \in \mathcal{P}}\right)$, then the above estimates may be patched together to yield (46). Such a partition of unity always exists. The point is that the magnitude $\left|d \eta_{P}\right|$ is roughly inversely proportional to the "width" of the overlaps $W_{P} \cap W_{Q}$ for all $Q \neq P$; two examples of these "widths", for $W_{Q_{1}} \cap W_{P}$ and $W_{G} \cap W_{Q_{1}}$, are indicated in Figure 20. However it is possible to choose $\left\{W_{P}\right\}_{P \in \mathcal{P}}$ so that these widths are arbitrarily large; this may involve enlarging $\widehat{X}_{0}$ through manipulation of the parameter $\mathbf{b}$ but there is plenty of room to do this since the boundary of $\widehat{X}$ is infinitely far away. Thus $\left\{\eta_{P}\right\}_{P \in \mathcal{P}}$ can be chosen with arbitrarily small derivative.

It remains to restrict our attention to a single $W_{P}$ and prove (47). 


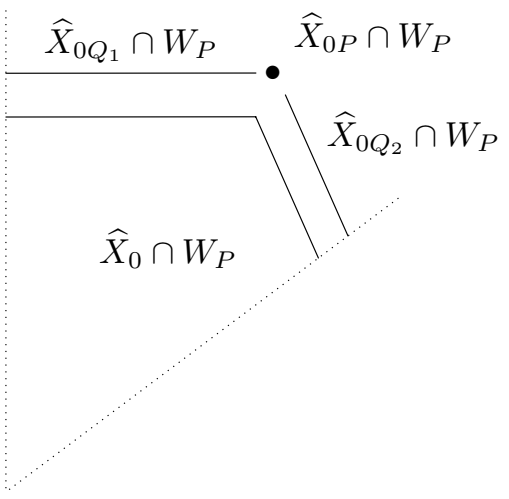

Figure 21. A single cylindrical set $W_{P}$

A section $\phi=\left(\phi_{Q}\right)_{Q \geq P}$ of $\mathcal{S}_{0}(\mathcal{M})$ over $\widehat{X}_{0} \cap W_{P}$ is composed of $\mathbb{E}_{Q}$-valued forms $\phi_{Q}$ which live on $\widehat{X}_{0 Q} \cap W_{P}$ as in Figure 21 above. We can apply harmonic projection to each $\phi_{Q}$ along the nilmanifold fibers $\mathcal{N}_{P}^{\prime Q}[\mathbf{8 1}]$ and obtain an $\left(\mathbb{H}\left(\mathfrak{n}_{P}^{Q} ; E_{Q}\right) \otimes \mathbb{C}_{\rho}\right)$ valued form on $\left(\left(-A_{P}^{Q} \cdot b_{P}\right) \times X_{P}\right) \cap W_{P}$; the factor $\mathbb{C}_{\rho}$ accounts for the volume of the nilmanifold fibers. However since we are seeking to prove the estimate (47) for compactly supported forms, it certainly suffices to consider more generally forms whose components have compact support anywhere in $\left({ }^{-} A_{P}^{Q} \cdot b_{P}\right) \times X_{P}$, which for simplicity we write as ${ }^{-} A_{P}^{Q} \times X_{P}$. The result is that we have unfolded the situation of Figure 19, which was a quotient by $\Gamma$, to the following quotient by $\Gamma_{P}$ :

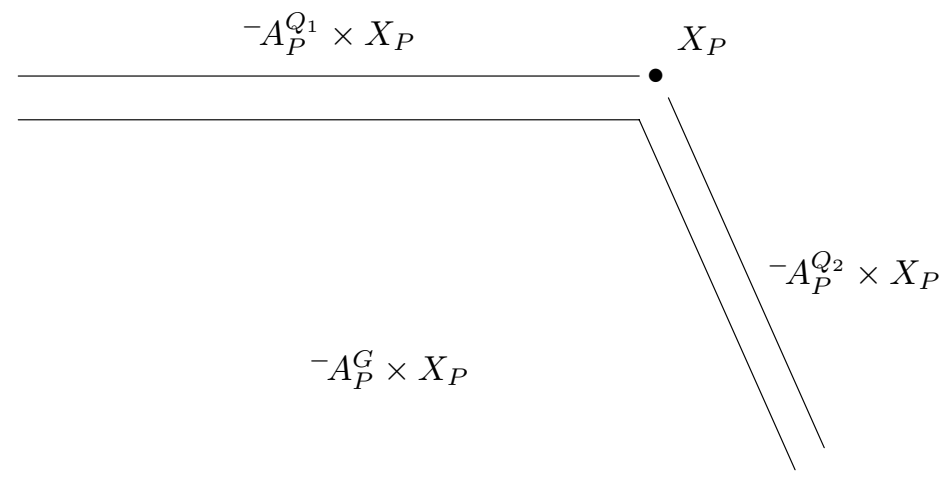


We need to establish the estimate (47) for compactly supported forms in the following unfolded complex:

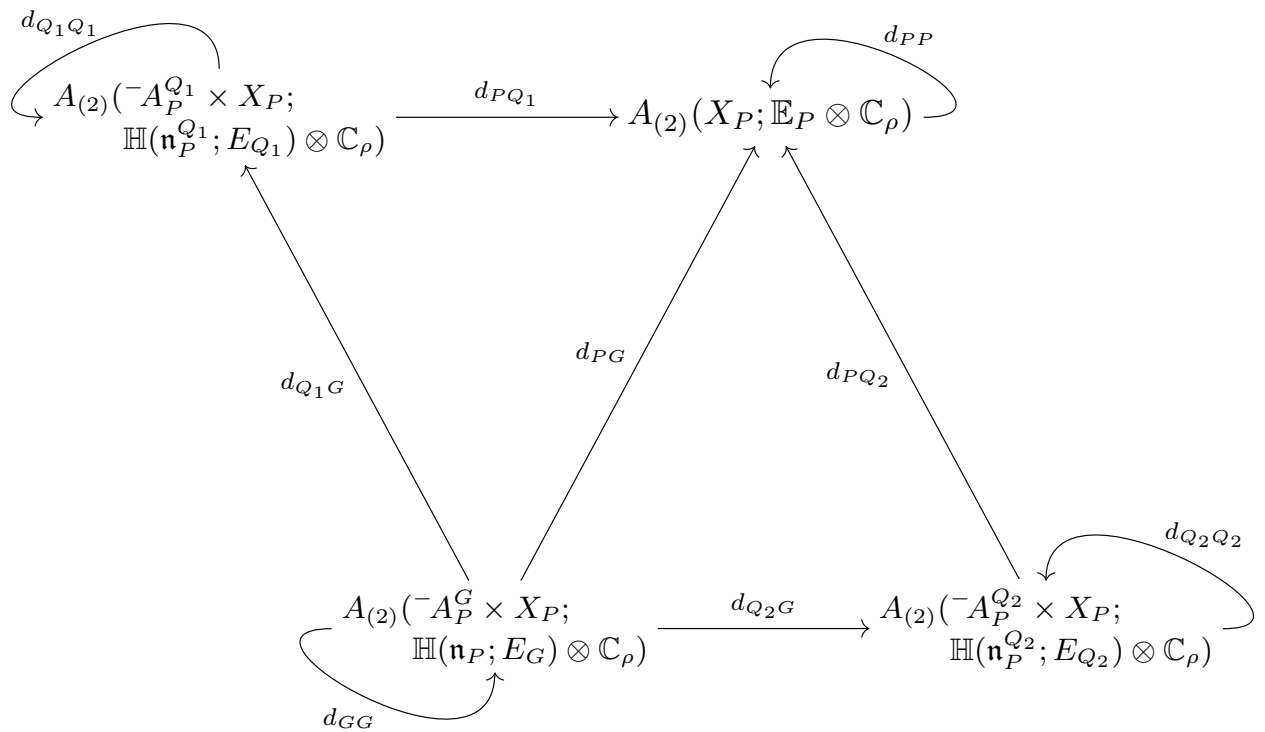

By Proposition 16.2 it suffices to show that the cohomology of this complex vanishes in degrees outside of $[c(\mathcal{M}), d(\mathcal{M})]$ and that the cohomology is Hausdorff in degree $c(\mathcal{M})$. It also suffices to do this with the coefficients replaced by their $V \otimes \mathbb{C}_{\rho^{-}}$ isotypical component for every irreducible representation $V$ of $L_{P}$.

Begin the calculation of the cohomology by forming the cohomology of each summand (for simplicity we assume that $f_{Q Q}=0$ for all $Q$, that is, $\mathcal{M}$ is reduced as in $\S 12.5)$ :

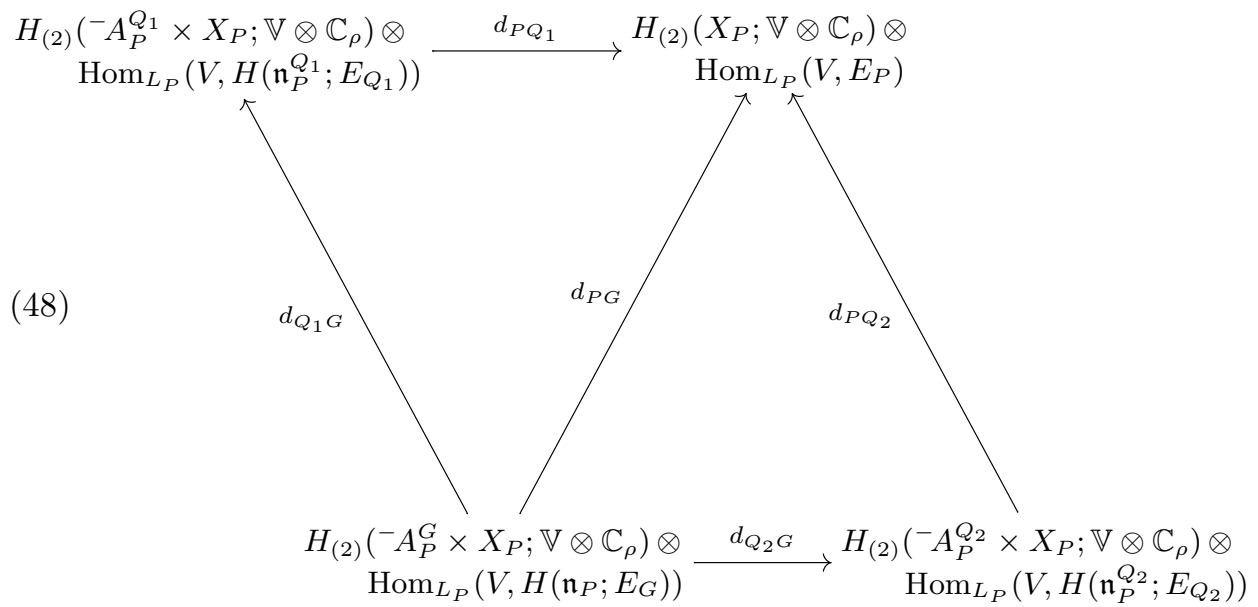

Consider a typical $L^{2}$-cohomology group that occurs in (48), say

$$
H_{(2)}\left({ }^{-} A_{P}^{Q} \times X_{P} ; \mathbb{V} \otimes \mathbb{C}_{\rho}\right)
$$

where $Q \geq P$. The locally constant sheaf $\mathbb{V} \otimes \mathbb{C}_{\rho}$ is constant along the ${ }^{-} A_{P}^{Q}$ factor however its metric is not; the coefficients add an exponential weight of $a^{-2\left(\xi_{V}+\rho\right)}$ 
to the $L^{2}$-norm integral. This weight factors as

$$
a^{-2\left(\xi_{V}+\rho\right)}=\prod_{\alpha \in \Delta_{P}^{Q}}\left(a^{-\beta_{\alpha}}\right)^{2\left(\xi_{V}+\rho, \alpha\right)}
$$

corresponding to the product decomposition

$$
{ }^{-} A_{P}^{Q} \cong[1, \infty)^{\Delta_{P}^{Q}}, \quad a \mapsto\left(a^{-\beta_{\alpha_{1}}}, \ldots, a^{\beta_{\alpha_{r}}}\right) .
$$

However the weighted $L^{2}$-cohomology $H_{(2)}\left([1, \infty) ; s^{k}\right)$ (where $s=a^{-\beta_{\alpha}}$ and $[1, \infty)$ has the multiplicatively invariant metric $\left.d s^{2} / s^{2}\right)$ vanishes for $k>0$ and is $\mathbb{C}$ for $k<0$. Thus whenever there exists $\alpha \in \Delta_{P}^{Q}$ such that $\left(\xi_{V}+\rho, \alpha\right)>0$, a Künnethtype argument [81] shows that (49) vanishes. Similarly if $\left(\xi_{V}+\rho, \alpha\right)<0$ for all $\alpha$ we can drop the ${ }^{-} A_{P}^{Q}$ factor in (49). The situation where $\left(\xi_{V}+\rho, \alpha\right)=0$ for some $\alpha$ can be handled by a slightly more delicate argument.

In order to illustrate the remainder of the proof, suppose that $\xi_{V}$ satisfies

$$
\left(\xi_{V}+\rho, \alpha_{1}\right)<0, \quad\left(\xi_{V}+\rho, \alpha_{2}\right)>0,
$$

where $\Delta_{P}=\left\{\alpha_{1}, \alpha_{2}\right\}$ and $\Delta_{P}^{Q_{i}}=\left\{\alpha_{i}\right\}$ for $i=1,2$. By the above arguments, equation (48) becomes

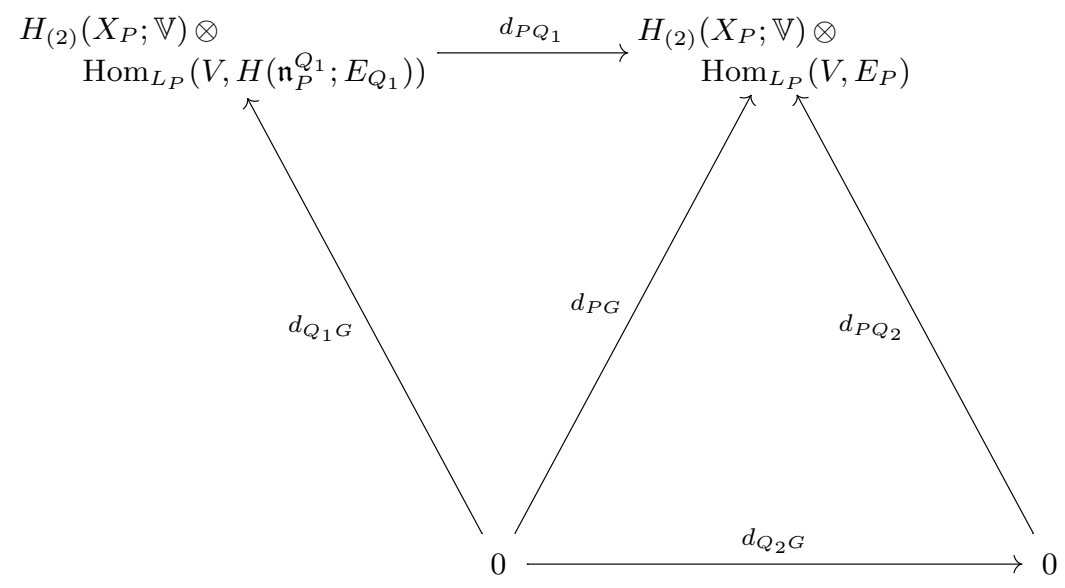

However $Q_{V}=Q_{V}^{\prime}=Q_{1}$ in this case so the total cohomology is (compare (32) in $\S 12.3)$

$$
H_{(2)}\left(X_{P} ; \mathbb{V}\right) \otimes \operatorname{Hom}_{L_{P}}\left(V, H\left(i_{P}^{*} \hat{\imath}_{Q_{V}} \mathcal{M}\right)\right) .
$$

The vanishing theorem for $L^{2}$-cohomology, Theorem 16.1, shows that the first factor of (51) vanishes unless $\left(\left.V\right|_{M_{P}}\right)^{*} \cong \overline{\left.V\right|_{M_{P}}}$ and the degree is in

$$
\left[\frac{1}{2}\left(\operatorname{dim} D_{P}-\operatorname{dim} D_{P}(V)\right), \frac{1}{2}\left(\operatorname{dim} D_{P}+\operatorname{dim} D_{P}(V)\right)\right]
$$

the cohomology is also Hausdorff in degree $\frac{1}{2}\left(\operatorname{dim} D_{P}-\operatorname{dim} D_{P}(V)\right)$. On the other hand, if $\left(\left.V\right|_{M_{P}}\right)^{*} \cong \overline{\left.V\right|_{M_{P}}}$ then the second factor of (51) vanishes unless $V \in \operatorname{SS}(\mathcal{M})$ and the degree is in

$$
[c(V ; \mathcal{M}), d(V ; \mathcal{M})]
$$

The sum of these two degree ranges gives the desired result.

This proves the vanishing theorem for the cohomology of an $\mathcal{L}$-module. 


\section{Micro-support of intersection cohomology}

When an $\mathcal{L}$-module is known explicitly the micro-support is not difficult to compute; for example, consider our calculation of $\operatorname{SS}\left(i_{G *} E\right)$ in $§ 13.2$. Unfortunately $\mathcal{I}_{p} \mathcal{C}(\widehat{X} ; E)$ is defined inductively and we do not have an explicit closed formula for its local cohomology. Furthermore the conjugate-self-contragredient condition in the definition of micro-support does not behave well under induction. Nonetheless we can prove the following combinatorial theorem [61, Corollary 17.2]:

TheOREM 18.1. Let $E$ be an irreducible $G$-module and let $p=m$ or $n$ be $a$ middle-perversity. Assume the $\mathbb{Q}$-root system of $G$ does not contain a factor of type $D_{n}, E_{n}$, or $F_{4}$. If $\left(\left.E\right|_{{ }^{\circ}}\right)^{*} \cong \overline{\left.E\right|^{{ }} G}$, then $\operatorname{SS}_{\text {ess }}\left(\mathcal{I}_{p} \mathcal{C}(\widehat{X} ; E)\right)=\{E\}$.

Actually we can describe the entire micro-support, not just the essential microsupport; see [61, Theorem 17.1] and (56), (57) below. In fact [61] treats the more general case in which $\left(\left.E\right|_{0} G\right)^{*} \cong \overline{\left.E\right|_{0} G}$ is not assumed. We expect that the condition on the $\mathbb{Q}$-root system will be able to be removed; the condition is satisfied in the Hermitian case or in the case of a real equal-rank Satake compactification.

What does the theorem say? Recall that an $L_{P}$-module $V$ belongs to $\operatorname{SS}_{\text {ess }}(\mathcal{M})$ if and only if

$$
\begin{aligned}
& \left(\left.V\right|_{M_{P}}\right)^{*} \cong \overline{\left.V\right|_{M_{P}}}, \text { and } \\
& H\left(i_{P}^{*} \hat{\imath}_{Q_{V}} \mathcal{M}\right)_{V} \longrightarrow H\left(i_{P}^{*} \hat{\imath}_{Q_{V}^{\prime}} \mathcal{M}\right)_{V} \text { is nonzero, }
\end{aligned}
$$

where we have defined $Q_{V}$ and $Q_{V}^{\prime}$ by

$$
\begin{aligned}
& \Delta_{P}^{Q_{V}}=\left\{\alpha \in \Delta_{P} \mid\left(\xi_{V}+\rho, \alpha\right)<0\right\}, \\
& \Delta_{P}^{Q_{V}^{\prime}}=\left\{\alpha \in \Delta_{P} \mid\left(\xi_{V}+\rho, \alpha\right) \leq 0\right\} .
\end{aligned}
$$

Since $i_{G}^{*} \hat{\imath}_{G}^{!} \mathcal{I}_{p} \mathcal{C}(\widehat{X} ; E)=E$, we certainly have $E \in \operatorname{SS}_{\text {ess }}\left(\mathcal{I}_{p} \mathcal{C}(\widehat{X} ; E)\right)$ if $\left(\left.E\right|_{0}\right)^{*} \cong$ $\overline{\left.E\right|_{0} G}$. The theorem is asserting that this is all: for every proper $P \in \mathcal{P}$ with parabolic rank $r(P)=1,2, \ldots$, there is no irreducible $L_{P}$-module $V \in \operatorname{SS}_{\text {ess }}\left(\mathcal{I}_{p} \mathcal{C}(\widehat{X} ; E)\right)$.

The only candidates for $V$ are the irreducible constituents of $H\left(\mathfrak{n}_{P} ; E\right)$, namely $V_{w(\lambda+\rho)-\rho}$ for $w \in W_{P}$. The assertion of the theorem amounts to a subtle relationship between the combinatorics of the Weyl element $w$, namely the minimal lengths in the cosets $W^{R} w$ for all $R \geq P$, and the geometry of $w(\lambda+\rho)$ vis $\grave{a}$ vis the roots $\alpha \in \Delta_{P}$. Since these matters play an important role in Goresky and MacPherson's topological trace formula [36] and in the representation of cohomology classes by Eisenstein series [69], it is likely that Theorem 18.1 will have applications beyond the Rapoport/Goresky-MacPherson conjecture.

The use of micro-support and of intersection cohomology is an efficient way to encode this relationship; a description of the result without these tools becomes unmanageable rapidly as the parabolic rank increases. However it is not difficult for low parabolic rank. In the following subsections we will sketch proofs for the parabolic rank 1 and 2 cases that will give some indication of the above relationship. Unfortunately these arguments do not generalize to higher parabolic rank; in particular, the condition on the $\mathbb{Q}$-root system begins to play a role with parabolic rank 4 . In the last subsection, we indicate some of the difficulties and outline how the general proof in [61] works.

From now on, we assume that $E$ is irreducible with highest weight $\lambda$. We will use Kostant's theorem and the notation introduced in $§ 13.2$. 
18.1. Parabolic rank 1. The simplest case is when $P$ is a maximal parabolic $\mathbb{Q}$-subgroup. Write $\Delta_{P}=\{\alpha\}$. For any $\mathcal{L}$-module $\mathcal{M}$, the expression $H\left(i_{P}^{*} \hat{l}_{Q}^{!} \mathcal{M}\right)$ is either the local cohomology at $P$ or the local cohomology supported at $P$ depending on whether $Q=G$ or $Q=P$ respectively. Given the definition of intersection cohomology (see in particular (38) in $\S 12.7)$, the cohomology of the link is $H\left(\mathfrak{n}_{P} ; E\right)$ and we have

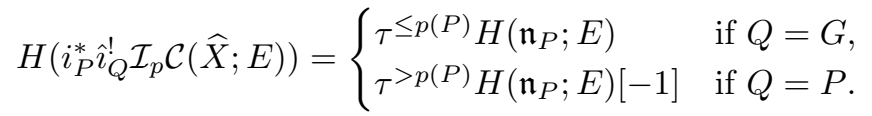

Recall that Kostant's theorem says that

$$
H\left(\mathfrak{n}_{P} ; E\right)=\bigoplus_{w \in W_{P}} H\left(\mathfrak{n}_{P} ; E\right)_{w}=\bigoplus_{w \in W_{P}} V_{w(\lambda+\rho)-\rho}[-\ell(w)] .
$$

Thus fix $V=V_{w(\lambda+\rho)-\rho}$ for some $w \in W_{P}$. Since $\xi_{V}+\rho=w(\lambda+\rho)$, the values of $Q_{V}$ and $Q_{V}^{\prime}$ are given by

- $(w(\lambda+\rho), \alpha)<0 \quad \Longrightarrow \quad Q_{V}=Q_{V}^{\prime}=G$,

- $(w(\lambda+\rho), \alpha)=0 \quad \Longrightarrow \quad Q_{V}=P, Q_{V}^{\prime}=G$,

- $(w(\lambda+\rho), \alpha)>0 \quad \Longrightarrow \quad Q_{V}=Q_{V}^{\prime}=P$.

On the other hand, it follows from (52) that the map in (ii)' is 0 precisely in the following cases:

- when $\ell(w)>p(P)$ and $Q_{V}=Q_{V}^{\prime}=G$,

- when $Q_{V}=P, Q_{V}^{\prime}=G$, and

- when $\ell(w) \leq p(P)$ and $Q_{V}=Q_{V}^{\prime}=P$.

By (7) the value of $p(P)$ is

$$
p(P)=p\left(\operatorname{dim} \mathfrak{n}_{P}+\# \Delta_{P}\right)= \begin{cases}\left\lfloor\left(\operatorname{dim} \mathfrak{n}_{P}-1\right) / 2\right\rfloor & p=m, \\ \left\lfloor\left(\operatorname{dim} \mathfrak{n}_{P}\right) / 2\right\rfloor & p=n .\end{cases}
$$

From the above facts, it is easy to see that the theorem in this case is equivalent to following proposition, which is a generalization of an old observation of Casselman [24] in the $\mathbb{R}$-rank 1 case; our proof uses Lemma 15.2.

Proposition 18.2. Let $E$ be an irreducible $G$-module with highest weight $\lambda$. Let $P$ be a maximal parabolic $\mathbb{Q}$-subgroup with $\Delta_{P}=\{\alpha\}$ and let $w \in W_{P}$. Assume that $\left(\left.V_{w(\lambda+\rho)-\rho}\right|_{M_{P}}\right)^{*} \cong \overline{\left.V_{w(\lambda+\rho)-\rho}\right|_{M_{P}}}$.

(i) If $(w(\lambda+\rho), \alpha) \leq 0$ then $\ell(w) \geq\left(\operatorname{dim} \mathfrak{n}_{P}\right) / 2$.

(ii) If $(w(\lambda+\rho), \alpha) \geq 0$ then $\ell(w) \leq\left(\operatorname{dim} \mathfrak{n}_{P}\right) / 2$.

Furthermore assume that $\left(\left.E\right|_{0_{G}}\right)^{*} \cong \overline{\left.E\right|_{{ } G}}$. Then

$$
\ell(w)=\left(\operatorname{dim} \mathfrak{n}_{P}\right) / 2 \quad \Longrightarrow \quad(w(\lambda+\rho), \alpha)=0 \text { for all } \alpha \in \Delta_{P} .
$$

Proof. The first two parts are simply Lemma 15.2 with the $\operatorname{dim} \mathfrak{n}_{P}(V)$ term omitted. For the final assertion, note that the proof of Lemma 15.2 together with the hypothesis $\ell(w)=\left(\operatorname{dim} \mathfrak{n}_{P}\right) / 2$ shows that for each root $\gamma$ in $\mathfrak{n}_{P \mathbb{C}}$, exactly one of $w^{-1} \gamma$ and $w^{-1} \bar{\gamma}=-w^{-1}(\theta \gamma)$ is negative, and that there are no real roots. The first statement implies that the positive system $w \Phi^{+}$is $\theta$-stable [61, Lemma 8.6]. The second statement, together with the Kostant-Sugiura classification of Cartan subalgebras $[\mathbf{4 4}],[\mathbf{7 2}],[\mathbf{7 3}]$, implies that $\mathfrak{h}$ is a fundamental Cartan subalgebra for $\mathfrak{g}$. Thus the operator $\tau_{G}$ equals $\theta$ as well, and hence the hypothesis $\left(\left.E\right|_{{ }_{0}}\right)^{*} \cong \overline{\left.E\right|^{\circ} G}$ is equivalent to $\left.w \lambda\right|_{\mathfrak{h}_{M_{P},-1}+\mathfrak{a}_{P}^{G}}=0$. (This is because $w \lambda$ is the highest weight of $E$ 
with respect to $w \Phi^{+}$.) Since $\left.w \rho\right|_{\mathfrak{h}_{M_{P},-1}+\mathfrak{a}_{P}^{G}}=0$ due to $w \Phi^{+}$being $\theta$-stable, we find that $\left.w(\lambda+\rho)\right|_{\mathfrak{a}_{P}^{G}}=0$ as desired.

18.2. Bidegree in $\boldsymbol{H}\left(\mathfrak{n}_{P} ; \boldsymbol{E}\right)$. For $P$ with parabolic rank $\geq 2$ we need a refinement of Kostant's theorem. Recall that for $P \leq Q$ we have an isomorphism

$$
H\left(\mathfrak{n}_{P} ; E\right) \cong H\left(\mathfrak{n}_{P}^{Q} ; H\left(\mathfrak{n}_{Q} ; E\right)\right) ;
$$

in particular $H\left(\mathfrak{n}_{P} ; E\right)$ is bigraded given $Q \geq P$. We write the bidegree as $\left(\ell^{Q}, \ell_{Q}\right)$ and define truncation by bidegree functors $\tau^{\ell_{Q} \leq n}$ and $\tau^{\ell_{Q}>n}$ on such bigraded modules. How is this structure reflected in the description of $H\left(\mathfrak{n}_{P} ; E\right)$ given by Kostant's theorem (53)?

Write

$$
W=W^{P} W_{P} \quad \text { and } \quad W=W^{Q} W_{Q}
$$

where $W^{P}$ is the Weyl group of $L_{P}$ and $W_{P}$ is the set of minimal length coset representatives of $W^{P} \backslash W$, and similarly for $Q$. Since $P / N_{Q}$ is a parabolic subgroup of $L_{Q}$, we also have a decomposition

$$
W^{Q}=W^{P} W_{P}^{Q}
$$

and thus $W=W^{P} W_{P}^{Q} W_{Q}$. One can show in fact that

$$
W_{P}=W_{P}^{Q} W_{Q}
$$

and hence for $w \in W_{P}$ we may write $w=w^{Q} w_{Q}$ corresponding to this decomposition. It is easy then to verify that

$$
H\left(\mathfrak{n}_{P} ; E\right)_{w} \cong H\left(\mathfrak{n}_{P}^{Q} ; H\left(\mathfrak{n}_{Q} ; E\right)_{w_{Q}}\right)_{w^{Q}} .
$$

So the bidegree of $H\left(\mathfrak{n}_{P} ; E\right)_{w}$ in $(54)$ is $\left(\ell^{Q}(w), \ell_{Q}(w)\right) \equiv\left(\ell\left(w^{Q}\right), \ell\left(w_{Q}\right)\right)$.

Recall that $\ell(w)$ may be interpreted as the number of positive $\mathbb{C}$-roots which $w^{-1}$ sends to negative roots. For $w \in W_{P}$, all such roots occur in $\mathfrak{n}_{P \mathbb{C}}$; the bidegree $\ell_{Q}(w)$ is the number of those roots occurring in $\mathfrak{n}_{Q \mathbb{C}}$ and the bidegree $\ell^{Q}(w)$ is the number of roots occurring in (a lift of) $\mathfrak{n}_{P \mathbb{C}}^{Q}$. In Figure 22 this is illustrated for two examples in the case $G=\mathrm{GL}_{n}(\mathbb{R})$, where positive roots may be identified with matrix positions above the diagonal. As one compares the various $\ell_{Q}(w)$, pictures such as these give helpful insight even for other groups, however one must be aware of the implicit assumption in the pictures that the Dynkin diagram of the root system is linear.

18.3. Parabolic rank 2. Now assume $r(P)=2$ and write $\Delta_{P}=\left\{\alpha_{1}, \alpha_{2}\right\}$ with $\Delta_{P}^{Q_{i}}=\left\{\alpha_{i}\right\}$ for $i=1,2$. The intersection cohomology $\mathcal{L}$-module is illustrated in Figure 15 of $\S 12.8$. In order to find a complex computing the link intersection cohomology at $P$, we start with $H\left(\mathfrak{n}_{P} ; E\right)$ and truncate it via a mapping cone in bidegree $\ell_{Q_{1}}>p\left(Q_{1}\right)$ and independently in bidegree $\ell_{Q_{2}}>p\left(Q_{2}\right)$. This yields the complex (40). We find that $H\left(\mathfrak{n}_{P} ; E\right)_{w}$ contributes a nonvanishing component to the link cohomology if neither truncation applies or if both truncations apply (in which case its degree is shifted up by 1$)$ :

$$
\tau^{\ell_{Q_{1}} \leq p\left(Q_{1}\right)} \tau^{\ell_{Q_{2}} \leq p\left(Q_{2}\right)} H\left(\mathfrak{n}_{P} ; E\right) \oplus \tau^{\ell_{Q_{1}}>p\left(Q_{1}\right)} \tau^{\ell_{Q_{2}}>p\left(Q_{2}\right)} H\left(\mathfrak{n}_{P} ; E\right)[-1] .
$$

See $\S 18.4$ below for a further discussion. 

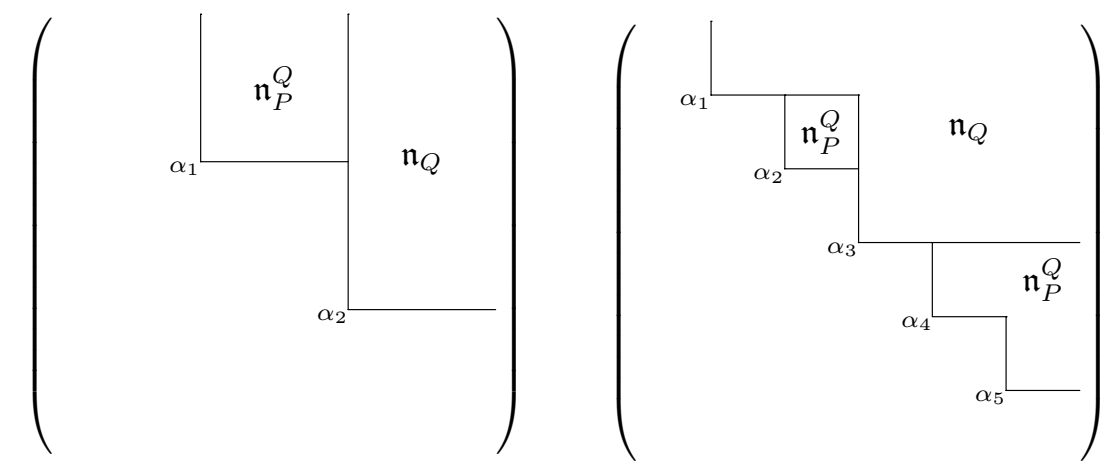
(a) $\Delta_{P}=\left\{\alpha_{1}, \alpha_{2}\right\}, \Delta_{P}^{Q}=\left\{\alpha_{1}\right\}$
(b) $\Delta_{P}=\left\{\alpha_{1}, \ldots, \alpha_{5}\right\}$ $\Delta_{P}^{Q}=\left\{\alpha_{2}, \alpha_{4}, \alpha_{5}\right\}$

FigURE 22. Unipotent radicals for various $P \leq Q$ in $\mathrm{GL}_{n}(\mathbb{R})$

This enables us to calculate $H\left(i_{P}^{*} \hat{l}_{Q}^{!} \mathcal{I}_{p} \mathcal{C}(\widehat{X} ; E)\right)$ if $Q=G$ or $Q=P$. For $Q=G$ we obtain the local intersection cohomology at $P$,

$$
\tau^{\leq p(P)}\left(\tau^{\ell_{Q_{1}} \leq p\left(Q_{1}\right)} \tau^{\ell_{Q_{2}} \leq p\left(Q_{2}\right)} H\left(\mathfrak{n}_{P} ; E\right) \oplus \tau^{\ell_{Q_{1}}>p\left(Q_{1}\right)} \tau^{\ell_{Q_{2}}>p\left(Q_{2}\right)} H\left(\mathfrak{n}_{P} ; E\right)[-1]\right) .
$$

For $Q=P$ we obtain the local intersection cohomology supported at $P$,

$$
\tau^{>p(P)}\left(\tau^{\ell_{Q_{1}} \leq p\left(Q_{1}\right)} \tau^{\ell_{Q_{2}} \leq p\left(Q_{2}\right)} H\left(\mathfrak{n}_{P} ; E\right) \oplus \tau^{\ell_{Q_{1}}>p\left(Q_{1}\right)} \tau^{\ell_{Q_{2}}>p\left(Q_{2}\right)} H\left(\mathfrak{n}_{P} ; E\right)[-1]\right)[-1] .
$$

In order to calculate micro-support, we need to gather some calculations and a generalization of Proposition 18.2. First, given that we are working with a middle perversity, we have

$p(P)=\left\{\begin{array}{ll}\left\lfloor\left(\operatorname{dim} \mathfrak{n}_{P}\right) / 2\right\rfloor & p=m, \\ \left\lfloor\left(\operatorname{dim} \mathfrak{n}_{P}+1\right) / 2\right\rfloor & p=n,\end{array} \quad\right.$ and $\quad p\left(Q_{i}\right)= \begin{cases}\left\lfloor\left(\operatorname{dim} \mathfrak{n}_{Q_{i}}-1\right) / 2\right\rfloor & p=m, \\ \left\lfloor\left(\operatorname{dim} \mathfrak{n}_{Q_{i}}\right) / 2\right\rfloor & p=n .\end{cases}$

Next it is helpful to note that if $k$ and $l$ are integers, then

$$
l \leq\lfloor k / 2\rfloor \quad \Longleftrightarrow \quad l \leq k / 2 \quad \text { and } \quad l>\lfloor k / 2\rfloor \quad \Longleftrightarrow l>k / 2 \text {; }
$$

the analogous formulas for $<$ and $\geq$ do not hold. Finally the generalization of Proposition 18.2 is:

Proposition 18.3. Let $E$ be an irreducible $G$-module with highest weight $\lambda$. Let $P$ be a parabolic $\mathbb{Q}$-subgroup and let $w \in W_{P}$. Assume that $\left(\left.V_{w(\lambda+\rho)-\rho}\right|_{P}\right)^{*} \cong$ $\overline{V_{w(\lambda+\rho)-\rho \mid M_{P}}}$.

(i) If $(w(\lambda+\rho), \alpha) \leq 0$ for all $\alpha \in \Delta_{P}$, then $\ell_{Q}(w) \geq\left(\operatorname{dim} \mathfrak{n}_{Q}\right) / 2$ for all $Q \geq P$.

(ii) If $(w(\lambda+\rho), \alpha) \geq 0$ for all $\alpha \in \Delta_{P}$, then $\ell_{Q}(w) \leq\left(\operatorname{dim} \mathfrak{n}_{Q}\right) / 2$ for all $Q \geq P$.

Furthermore assume that $\left(\left.E\right|_{0} G\right)^{*} \cong \overline{E \mid{ }^{\circ} G}$ and that the hypotheses of (i) or (ii) hold. Then

$$
\ell(w)=\left(\operatorname{dim} \mathfrak{n}_{P}\right) / 2 \quad \Longrightarrow \quad(w(\lambda+\rho), \alpha)=0 \text { for all } \alpha \in \Delta_{P} .
$$

Sketch of PROOF. For every $A_{P}$-weight $\alpha$ in $\mathfrak{n}_{Q}$ (not necessarily simple), the argument we gave for Lemma 15.2 may be applied to the $\mathbb{C}$-roots $\gamma$ for which 
$\left.\gamma\right|_{\mathfrak{a}_{P}}=\alpha$. The result is an estimate on the contribution to $\ell_{Q}(w)$ from such roots. The sum of these estimates over all $A_{P}$-weights in $\mathfrak{n}_{Q}$ yields the proposition. The last assertion follows as in Proposition 18.2.

Consider $V=V_{w(\lambda+\rho)-\rho}$ for some $w \in W_{P}$ and assume that $\left(\left.V\right|_{M_{P}}\right)^{*} \cong \overline{\left.V\right|_{M_{P}}}$. In the case $Q_{V}=P$, we have $(w(\lambda+\rho), \alpha) \geq 0$ for all $\alpha \in \Delta_{P}$. It follows from the proposition and the preceding calculations that $H\left(i_{P}^{*} \hat{l}_{Q_{V}}^{\prime} \mathcal{I}_{p} \mathcal{C}(\widehat{X} ; E)\right)_{V}$ is 0 unless $\ell(w)=\left(\operatorname{dim} \mathfrak{n}_{P}\right) / 2$, in which case $(w(\lambda+\rho), \alpha)=0$ for all $\alpha \in \Delta_{P}$ and thus $Q_{V}^{\prime}=G$. Conversely if $Q_{V}^{\prime}=G$ then $(w(\lambda+\rho), \alpha) \leq 0$ for all $\alpha \in \Delta_{P}$ and it follows that either $H\left(i_{P}^{*} \hat{\imath}_{Q_{V}^{\prime}}^{\prime} \mathcal{I}_{p} \mathcal{C}(\widehat{X} ; E)\right)_{V}=0$ or $\ell(w)=\left(\operatorname{dim} \mathfrak{n}_{P}\right) / 2$ and $Q_{V}=P$.

Thus for any $V$ which satisfies $Q_{V}$ or $Q_{V}^{\prime}$ equal to either $P$ or $G$ and which can conceivably contribute to micro-support, we have $Q_{V}=P, Q_{V}^{\prime}=G, \ell(w)=$ $\left(\operatorname{dim} \mathfrak{n}_{P}\right) / 2$, and

$$
\begin{aligned}
H\left(i_{P}^{*} \hat{l}_{Q_{V}}^{!} \mathcal{I}_{p} \mathcal{C}(\widehat{X} ; E)\right)_{V} & = \begin{cases}V[-\ell(w)-2] & \text { if } p=m, \\
0 & \text { if } p=n .\end{cases} \\
H\left(i_{P}^{*} \hat{l}_{Q_{V}^{\prime}}^{!} \mathcal{I}_{p} \mathcal{C}(\widehat{X} ; E)\right)_{V} & = \begin{cases}0 & \text { if } p=m, \\
V[-\ell(w)] & \text { if } p=n .\end{cases}
\end{aligned}
$$

Such $V$ belong to $\operatorname{SS}\left(\mathcal{I}_{p} \mathcal{C}(\widehat{X} ; E)\right)$ but obviously do not contribute to the essential micro-support; we call them fundamental since they occur if and only if $P$ contains a fundamental parabolic $\mathbb{R}$-subgroup of $G[\mathbf{1 3}]$, [61, Lemma 8.8]. (Recall that a parabolic $\mathbb{R}$-subgroup is fundamental if a fundamental Cartan subalgebra for its Levi quotient lifts to a fundamental Cartan subalgebra for $G$.)

Unlike the parabolic rank 1 case, there are two more cases of $H\left(i_{P}^{*} \hat{\imath}_{Q} \mathcal{I}_{p} \mathcal{C}(\widehat{X} ; E)\right)$ to consider, namely when $Q=Q_{1}$ or $Q=Q_{2}$. Since the two cases are identical after relabeling, we focus on $Q=Q_{1}$. Use the complex $\hat{\imath}_{P}^{*} \hat{\imath}_{Q}^{!} \mathcal{M}$ of (32) in $\S 12.3$ applied to the $\mathcal{L}$-module $\mathcal{M}=\mathcal{I}_{p} \mathcal{C}(\widehat{X} ; E)$ pictured in Figure 15 . We compute that

$$
\begin{aligned}
H\left(i_{P}^{*} \hat{\imath}_{Q_{1}}^{!} \mathcal{I}_{p} \mathcal{C}(\widehat{X} ; E)\right)= & \tau^{\ell_{Q_{1}}>p\left(Q_{1}\right)} \tau^{\ell_{Q_{2}} \leq p\left(Q_{2}\right)} H\left(\mathfrak{n}_{P} ; E\right)[-1] \oplus \\
& \tau^{\leq p(P)}\left(\tau^{\ell_{Q_{1}}>p\left(Q_{1}\right)} \tau^{\ell_{Q_{2}}>p\left(Q_{2}\right)} H\left(\mathfrak{n}_{P} ; E\right)[-1]\right) \oplus \\
& \tau^{>p(P)} \tau^{\ell_{Q_{1}} \leq p\left(Q_{1}\right)} \tau^{\ell_{Q_{2}} \leq p\left(Q_{2}\right)} H\left(\mathfrak{n}_{P} ; E\right)[-1] .
\end{aligned}
$$

The first term are those classes in $\tau^{\ell_{Q_{1}}>p\left(Q_{1}\right)} H\left(\mathfrak{n}_{P} ; E\right)[-1]$ which map to 0 in the link cohomology, the next term are those classes which map to nonzero elements in the link cohomology which are not truncated at $P$, and the third term are those link cohomology classes being truncated at $P$ which do not come from $\tau^{\ell_{Q_{1}}>p\left(Q_{1}\right)} H\left(\mathfrak{n}_{P} ; E\right)[-1]$.

We can now handle $V$ such that $Q_{V}=Q_{V}^{\prime}=Q_{1}$. In this case

$$
H\left(i_{P}^{*} \hat{l}_{Q_{1}}^{!} \mathcal{I}_{p} \mathcal{C}(\widehat{X} ; E)\right)_{V}=0
$$

by the proposition below (which is actually slightly stronger than necessary). Consequently $V$ cannot be in $\operatorname{SS}\left(\mathcal{I}_{p} \mathcal{C}(\widehat{X} ; E)\right)$, much less the essential micro-support. The case where $Q_{V}=Q_{V}^{\prime}=Q_{2}$ is treated similarly.

Proposition 18.4. Let $E$ be an irreducible $G$-module with highest weight $\lambda$. Let $P$ be a parabolic $\mathbb{Q}$-subgroup with $\Delta_{P}=\left\{\alpha_{1}, \alpha_{2}\right\}$ and let $w \in W_{P}$. Assume that 


$$
\begin{aligned}
\left(\left.V_{w(\lambda+\rho)-\rho}\right|_{M_{P}}\right)^{*} \cong & \overline{\left.V_{w(\lambda+\rho)-\rho}\right|_{M_{P}}} \text { and that } \\
& \left(w(\lambda+\rho), \alpha_{1}\right) \leq 0 \text { and }\left(w(\lambda+\rho), \alpha_{2}\right) \geq 0 .
\end{aligned}
$$

(i) If $\ell_{Q_{1}}(w) \geq\left(\operatorname{dim} \mathfrak{n}_{Q_{1}}\right) / 2$ then $\ell(w) \geq\left(\operatorname{dim} \mathfrak{n}_{P}\right) / 2$.

(ii) If $\ell_{Q_{2}}(w) \leq\left(\operatorname{dim} \mathfrak{n}_{Q_{2}}\right) / 2$ then $\ell(w) \leq\left(\operatorname{dim} \mathfrak{n}_{P}\right) / 2$.

If either hypothesis is a strict inequality, the corresponding conclusion is also strict.

Sketch of Proof. Apply Proposition 18.2 to the group $L_{Q_{1}}$ with the representation $E_{Q_{1}}=H^{\ell_{Q_{1}}(w)}\left(\mathfrak{n}_{Q_{1}} ; E\right)_{w_{Q_{1}}}$, the maximal parabolic subgroup $P / N_{Q_{1}}$, and the Weyl element $w^{Q_{1}} \in W_{P}^{Q_{1}}$. If $\mu$ is the highest weight of $E_{Q_{1}}$, the representation $H^{\ell^{Q_{1}}}(w)\left(\mathfrak{n}_{P}^{Q_{1}} ; E_{Q_{1}}\right)_{w^{Q_{1}}}=V_{w^{Q_{1}}(\mu+\rho)-\rho}$ is identical with $V_{w(\lambda+\rho)-\rho}$ and hence satisfies the conjugate self-contragredient condition. It follows from the proposition that $\ell^{Q_{1}}(w) \geq\left(\operatorname{dim} \mathfrak{n}_{P}^{Q_{1}}\right) / 2$ which proves (i) since $\ell(w)=\ell_{Q_{1}}(w)+\ell^{Q_{1}}(w)$. Part (ii) follows similarly.

REMARK. Whereas the conjugate self-contragredience of $H\left(\mathfrak{n}_{P} ; E\right)_{w}$ trivially implied the conjugate self-contragredience of $H\left(\mathfrak{n}_{P}^{Q_{1}} ; E_{Q_{1}}\right)_{w^{Q_{1}}}$ in the proof above, it does not in general imply the conjugate self-contragredience of $H\left(\mathfrak{n}_{Q_{1}} ; E\right)_{w_{Q_{1}}}$. As we will indicate in $\S 18.5$ below, this is a fundamental difficulty in formulating a proof that applies to arbitrary parabolic rank. The natural approach would be to obtain information regarding the cohomology of the link by applying the theorem inductively to larger parabolic subgroups; this fails since the self-contragredience condition is not preserved under the induction which replaces $w$ by $w_{R}$ for $R \geq P$.

18.4. Geometric interpretation of link cohomology. Although the calculation of link intersection cohomology preceding (55) is trivial, it is helpful when considering higher parabolic rank to view it geometrically. Thus we digress to indicate this point of view. Recall that $\left|\Delta_{P}\right|$ denotes the closed $(r(P)-1)$-simplex with vertices $\alpha \in \Delta_{P}$; this is a stratified space with strata $\left|\Delta_{P}^{Q}\right|^{\circ}$ indexed by $Q>P$. For $w \in W_{P}$, define the $w$-shifted perversity

$$
p_{w}(Q) \equiv p(Q)-\ell_{Q}(w)=p\left(\operatorname{dim} \mathfrak{n}_{Q}+\# \Delta_{Q}\right)-\ell_{Q}(w), \quad Q \geq P
$$

we view this as a generalized perversity for the space $\left|\Delta_{P}\right|$ and so the intersection cohomology $I_{p_{w}} H\left(\left|\Delta_{P}\right| ; \mathbb{Z}\right)$ is defined. The $H\left(\mathfrak{n}_{P} ; E\right)_{w}$-isotypical component of the complex $i_{P}^{*} j_{P *} j_{P}^{*} \mathcal{I}_{p} \mathcal{C}(\widehat{X} ; E)$ computing link intersection cohomology can be expressed as

$$
H\left(\mathfrak{n}_{P} ; E\right)_{w} \otimes I_{p_{w}} C\left(\left|\Delta_{P}\right| ; \mathbb{Z}\right) ;
$$

we take this as defining $I_{p_{w}} C\left(\left|\Delta_{P}\right| ; \mathbb{Z}\right)$. The corresponding isotypical component of the link intersection cohomology is

$$
H\left(\mathfrak{n}_{P} ; E\right)_{w} \otimes I_{p_{w}} H\left(\left|\Delta_{P}\right| ; \mathbb{Z}\right) .
$$

The isotypical components of $H\left(i_{P}^{*} \mathcal{I}_{p} \mathcal{C}(\widehat{X} ; E)\right)$, the local intersection cohomology, are expressed similarly using $I_{p_{w}} H\left(c\left(\left|\Delta_{P}\right|\right) ; \mathbb{Z}\right)$. Here $c\left(\left|\Delta_{P}\right|\right)$ is the cone on $\left|\Delta_{P}\right|$, a stratified space with strata $c\left(\left|\Delta_{P}^{Q}\right|\right)^{\circ}$ indexed by $Q \geq P$. (The stratum associated to $Q=P$ is the vertex of the cone.)

For example, in the case of parabolic rank two, $\left|\Delta_{P}\right|$ is a closed 1-simplex with vertices $\left\{\alpha_{1}, \alpha_{2}\right\}$. Let $B_{w} \subseteq\left\{\alpha_{1}, \alpha_{2}\right\}$ denote the set of vertices where a degree 0 


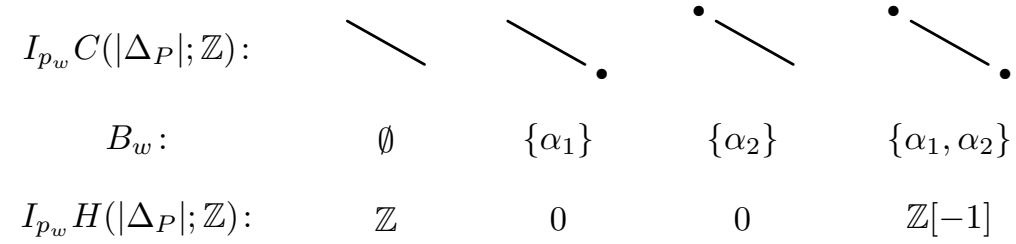

FIGURE 23. The intersection cohomology $I_{p_{w}} H\left(\left|\Delta_{P}\right| ; \mathbb{Z}\right)$ in parabolic rank 2

class on the interior $\left|\Delta_{P}\right|^{\circ}$ would be truncated according to $p_{w}$, that is,

$$
B_{w} \equiv \begin{cases}\emptyset & \text { if } \ell_{Q_{1}}(w) \leq p\left(Q_{1}\right), \ell_{Q_{2}}(w) \leq p\left(Q_{2}\right), \\ \left\{\alpha_{1}\right\} & \text { if } \ell_{Q_{1}}(w)>p\left(Q_{1}\right), \ell_{Q_{2}}(w) \leq p\left(Q_{2}\right), \\ \left\{\alpha_{2}\right\} & \text { if } \ell_{Q_{1}}(w) \leq p\left(Q_{1}\right), \ell_{Q_{2}}(w)>p\left(Q_{2}\right), \\ \left\{\alpha_{1}, \alpha_{2}\right\} & \text { if } \ell_{Q_{1}}(w)>p\left(Q_{1}\right), \ell_{Q_{2}}(w)>p\left(Q_{2}\right) .\end{cases}
$$

Then $I_{p_{w}} C\left(\left|\Delta_{P}\right| ; \mathbb{Z}\right)$ is the complex $\mathbb{Z} \rightarrow \mathbb{Z}^{\# B_{w}}$ (compare (40) in $\S 12.8$ ).

We graphically represent $I_{p_{w}} C\left(\left|\Delta_{P}\right| ; \mathbb{Z}\right)$ in Figure 23 along with the value of $I_{p_{w}} H\left(\left|\Delta_{P}\right| ; \mathbb{Z}\right)$ by placing a dot near $\alpha_{i}$ if truncation is taking place there. The corresponding diagrams for parabolic rank 3 are given in Figure 24; here $\left|\Delta_{P}\right|$ is a 2 -simplex and we draw a line near an edge if truncation of the degree 0 class on the interior is occurring, and we draw a dot near a vertex if the degree 0 or 1 class on its link is being truncated.

Properly speaking, we should decorate the dots and lines in these figures with the degree $p_{w}(Q)$ above which we truncate. However the cohomology potentially being truncated occurs only in one degree (namely 0 or 1), so simply indicating whether or not it is truncated is sufficient. The same shortcut can be taken in parabolic rank 4, but can fail beginning with parabolic rank 5 . The point is that if $P$ has parabolic rank 5 , a vertex in $\left|\Delta_{P}\right|$ corresponds to a subgroup of parabolic rank 4 and, as we will indicate in the next paragraph, the isotypical components of link intersection cohomology at a parabolic rank 4 subgroup can live in several degrees.

Incidentally, the figures show that in both parabolic rank 2 and 3, the isotypical components (58) of link intersection cohomology (55) occur in a single degree. This phenomenon does not persist however; it can fail for parabolic rank $\geq 4$. For example, if $P$ has parabolic rank 4 and thus $\left|\Delta_{P}\right|$ is a 3 -dimensional simplex, there are two configurations that lead to cohomology in more one degree: one can have truncation at all 4 faces as well as at 3 non-coplanar edges or dually one can have truncation at all 4 vertices and at 3 edges bounding a face. The resulting complex in the first case is $0 \rightarrow \mathbb{Z} \rightarrow \mathbb{Z}^{4} \rightarrow \mathbb{Z}^{3} \rightarrow \mathbb{Z}$ which yields cohomology in degrees 1 and 2 . These configurations can actually arise from Weyl group elements. ${ }^{3}$

\footnotetext{
${ }^{3}$ Among the real symplectic groups one must wait until $G=\mathrm{Sp}_{20}(\mathbb{R})$ to see them. For this group, the parabolic $P$ with $\Delta^{P}=\left\{\alpha_{2}, \alpha_{4}, \alpha_{5}, \alpha_{7}, \alpha_{8}, \alpha_{9}\right\}$ has three elements $w \in W_{P}$ which yield the first configuration above; one of them is

$w=s_{3} s_{2} s_{1} s_{6} s_{5} s_{4} s_{3} s_{2} s_{7} s_{6} s_{5} s_{4} s_{3} s_{8} s_{10} s_{9} s_{8} s_{7} s_{6} s_{5} s_{4}$

$s_{10} s_{9} s_{8} s_{7} s_{6} s_{5} s_{10} s_{9} s_{8} s_{7} s_{6} s_{10} s_{9} s_{8} s_{7} s_{10} s_{9} s_{8} s_{10} s_{9} s_{10}$.
} 


$$
I_{p_{w}} C\left(\left|\Delta_{P}\right| ; \mathbb{Z}\right):
$$

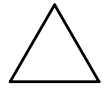

$I_{p_{w}} H\left(\left|\Delta_{P}\right| ; \mathbb{Z}\right):$

$I_{p_{w}} C\left(\left|\Delta_{P}\right| ; \mathbb{Z}\right):$

$I_{p_{w}} H\left(\left|\Delta_{P}\right| ; \mathbb{Z}\right):$

$I_{p_{w}} C\left(\left|\Delta_{P}\right| ; \mathbb{Z}\right):$

$I_{p_{w}} H\left(\left|\Delta_{P}\right| ; \mathbb{Z}\right):$
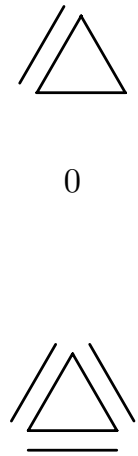

$\mathbb{Z}$

0

$\mathbb{Z}^{2}[-1]$

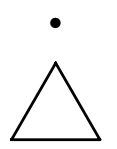

0

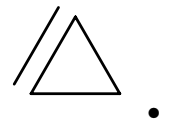

$\mathbb{Z}[-1]$

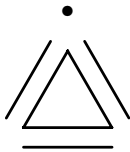

$\mathbb{Z}[-1]$

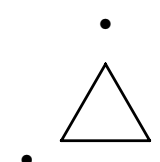

$\mathbb{Z}[-1]$

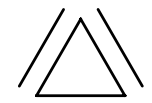

$\mathbb{Z}[-1]$

0

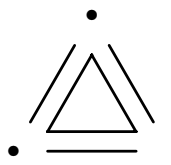

0

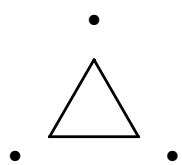

$\mathbb{Z}^{2}[-1]$

Figure 24. The intersection cohomology $I_{p_{w}} H\left(\left|\Delta_{P}\right| ; \mathbb{Z}\right)$ in parabolic rank 3. (Configurations differing from previous ones by a rotation are omitted.)

Finally, note that in all the illustrated examples, the intersection cohomology groups $I_{p_{w}} H\left(\left|\Delta_{P}\right| ; \mathbb{Z}\right)$ can be viewed simply as relative cohomology groups $H\left(\left|\Delta_{P}\right|, C_{w}\right)$ for a certain subset $C_{w} \subseteq \partial\left|\Delta_{P}\right|$. Namely, $C_{w}$ is the union of the boundary strata at which truncation is taking place. Again this interpretation continues to hold in parabolic rank 4 but fails (at least with the above definition of $C_{w}$ ) beginning with parabolic rank 5 .

18.5. Parabolic rank $\geq 3$. If $Q_{V}$ or $Q_{V}^{\prime}$ is equal to $P$ or $G$, the argument already given using Proposition 18.3 applies to $P$ with any parabolic rank to show that $V=H^{\ell(w)}\left(\mathfrak{n}_{P} ; E\right)_{w}$ can only contribute to the micro-support when it is fundamental and can never contribute to the essential micro-support [61, Proposition 17.7].

It remains to consider $V$ such that $P<Q_{V} \leq Q_{V}^{\prime}<G$. In this case there is a direct though tedious proof for parabolic rank 3 that $V$ is not in the micro-support; it uses Figure 24 and the same ideas as in the parabolic rank 2 case. Obviously this approach does not lend itself to a general argument; it requires a case-by-case calculation of $H\left(i_{P}^{*} \hat{l}_{Q}^{!} \mathcal{I}_{p} \mathcal{C}(\widehat{X} ; E)\right)_{V}$ for all the situations illustrated in Figure 24 .

Unfortunately in this example, $\ell(w)=42$, $\operatorname{dim} \mathfrak{n}_{P}=90$, and hence $p_{w}(P)=4$ or $4 \frac{1}{2}$. This means that both of the link cohomology classes in degrees 1 and 2 will not be truncated. We expect that there are examples in higher rank where only part of the link cohomology is truncated. 
We will now outline the ingredients for a general proof. In the case of parabolic rank 3, a complete proof can be constructed from what we present here; in general one must consult $[\mathbf{6 1}]$. Recall that we wish to show that

$$
H\left(i_{P}^{*} \hat{\imath}_{Q} \mathcal{I}_{p} \mathcal{C}(\widehat{X} ; E)\right)_{V}=0
$$

under the hypotheses

(i) $V=H^{\ell(w)}\left(\mathfrak{n}_{P} ; E\right)_{w}$ is conjugate self-contragredient when restricted to $M_{P}$.

(ii) $P<Q_{V} \leq Q \leq Q_{V}^{\prime}<G$.

We can re-express

$$
H\left(i_{P}^{*} \hat{\imath}_{Q}^{!} \mathcal{I}_{p} \mathcal{C}(\widehat{X} ; E)\right)_{V} \cong H\left(\mathfrak{n}_{P} ; E\right)_{w} \otimes I_{p_{w}} H_{c\left(\left|\Delta_{P}^{Q}\right|\right)}
$$

[61, Proposition 17.4], where we use the convenient shorthand

$$
I_{p_{w}} H_{c\left(\left|\Delta_{P}^{Q}\right|\right)} \equiv I_{p_{w}} H\left(c\left(\left|\Delta_{P}\right|\right), c\left(\left|\Delta_{P}\right|\right) \backslash c\left(\left|\Delta_{P}^{Q}\right|\right) ; \mathbb{Z}\right)
$$

for the generalized perversity intersection cohomology supported on $c\left(\left|\Delta_{P}^{Q}\right|\right)$. (We will henceforth omit the coefficients $\mathbb{Z}$ from the notation.) Thus (59) may be replaced by

$$
I_{p_{w}} H_{c\left(\left|\Delta_{P}^{Q}\right|\right)}=0 .
$$

We exploit the local calculation of intersection cohomology (8) at the vertex of the cone,

$$
I_{p_{w}} H^{j}\left(c\left(\left|\Delta_{P}\right|\right)\right)= \begin{cases}I_{p_{w}} H^{j}\left(\left|\Delta_{P}\right|\right) & \text { for } j \leq p_{w}(P) \\ 0 & \text { for } j>p_{w}(P) .\end{cases}
$$

From this and the exact sequence of the pair, one can show that (60) is equivalent to

$$
\begin{array}{ll}
I_{p_{w}} H^{j-1}\left(\left|\Delta_{P}\right| \backslash\left|\Delta_{P}^{Q}\right|\right)=0 & \text { for } j>p_{w}(P)+1, \\
\operatorname{Im}\left(I_{p_{w}} H^{p_{w}(P)}\left(\left|\Delta_{P}\right| \backslash\left|\Delta_{P}^{Q}\right|\right) \longrightarrow I_{p_{w}} H_{\left|\Delta_{P}^{Q}\right|}^{p_{w}(P)+1}\right)=0 & \text { for } j=p_{w}(P)+1, \\
I_{p_{w}} H_{\left|\Delta_{P}^{Q}\right|}^{j}=0 & \text { for } j<p_{w}(P)+1 .
\end{array}
$$

We will focus on proving (62a). It turns out that the argument also shows that the domain of the map in $(62 \mathrm{~b})$ vanishes in half of the cases. Then a dual argument proves (62c) and also that the range of the map in (62b) vanishes in the other half of the cases.

There are two spectral sequences [61, Lemma 3.7] we use that abut to

$$
I_{p_{w}} H^{j-1}\left(\left|\Delta_{P}\right| \backslash\left|\Delta_{P}^{Q}\right|\right) .
$$

First some notation. Let $S>P$ be the parabolic $\mathbb{Q}$-subgroup complementary to $Q$ relative to $P$, that is, such that $\Delta_{P}^{S}=\Delta_{P} \backslash \Delta_{P}^{Q}$. For each $\alpha \in \Delta_{P}$, let $U_{\alpha}$ be the open star neighborhood of the corresponding vertex of $\left|\Delta_{P}\right|$; note that $U_{\alpha}=$ $\bigcup_{\alpha \in \Delta_{P}^{R} \subseteq \Delta_{P}}\left|\Delta_{P}^{R}\right|^{\circ}$. Set $U_{R} \equiv \bigcap_{\alpha \in \Delta_{P}^{R}} U_{\alpha}$ for $R>P$, the open star neighborhood of $\left|\Delta_{P}^{R}\right|^{\circ}$, and define a parabolic $\mathbb{Q}$-subgroup $Q \vee R$ by $\Delta_{P}^{Q \vee R}=\Delta_{P}^{Q} \cup \Delta_{P}^{R}$. The 
$\alpha_{1}$

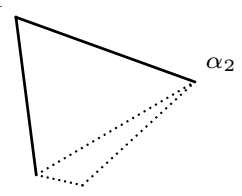

$U_{\alpha_{1}}$ $\alpha$

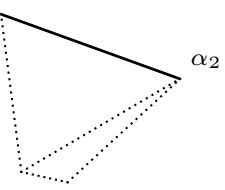

$U_{\alpha_{1}} \cap U_{\alpha_{2}}$ $\alpha_{1}$

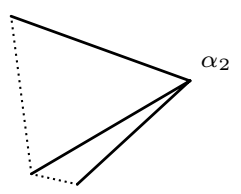

$U_{\alpha_{2}}$

Figure 25. The Mayer-Vietoris spectral sequence for $I_{p_{w}} H\left(\left|\Delta_{P}\right| \backslash\right.$ $\left.\left|\Delta_{P}^{Q}\right|\right)$, where $\Delta_{P}^{Q}=\left\{\alpha_{3}, \alpha_{4}\right\}$

$\alpha_{1}$

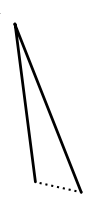

$\alpha_{1}$

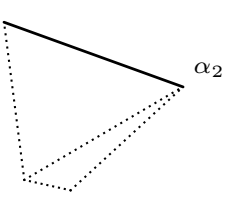

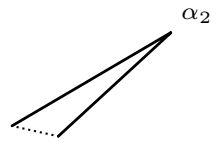

$U_{\alpha_{1}} \cap\left|\Delta_{P}^{Q} \cup\left\{\alpha_{1}\right\}\right| \quad U_{\alpha_{1}} \cap U_{\alpha_{2}} \cap\left|\Delta_{P}^{Q} \cup\left\{\alpha_{1}, \alpha_{2}\right\}\right| \quad U_{\alpha_{2}} \cap\left|\Delta_{P}^{Q} \cup\left\{\alpha_{2}\right\}\right|$

Figure 26. The Fary spectral sequence for $I_{p_{w}} H\left(\left|\Delta_{P}\right| \backslash\left|\Delta_{P}^{Q}\right|\right)$, where $\Delta_{P}^{Q}=\left\{\alpha_{3}, \alpha_{4}\right\}$

Mayer-Vietoris spectral sequence for the open cover $\left\{U_{\alpha}\right\}_{\alpha \in \Delta_{P}^{S}}$ of $\left|\Delta_{P}\right| \backslash\left|\Delta_{P}^{Q}\right|$ has $^{4}$

$$
E_{1}^{p, j-1-p}=\bigoplus_{\substack{P<R \leq S \\ \# \Delta_{P}^{R}=p+1}} I_{p_{w}} H^{j-1-p}\left(U_{R}\right) \cong \bigoplus_{\substack{P<R \leq S \\ \# \Delta_{P}^{R}=p+1}} I_{p_{w}} H^{j-1-p}\left(c\left(\left|\Delta_{R}\right|\right)\right) .
$$

See Figure 25. On the other hand, the projection from $\left|\Delta_{P}^{Q}\right|$ defines a fibration $\left|\Delta_{P}\right| \backslash\left|\Delta_{P}^{Q}\right| \rightarrow\left|\Delta_{P}^{S}\right|$. For $P<R \leq S$, the inverse image of the stratum $\left|\Delta_{P}^{R}\right|^{\circ} \subseteq\left|\Delta_{P}^{S}\right|$ is $U_{R} \cap\left|\Delta_{P}^{Q \vee R}\right| \subseteq\left|\Delta_{P}\right| \backslash\left|\Delta_{P}^{Q}\right|$. The associated Fary spectral sequence has

$$
E_{1}^{-p, j-1+p}=\bigoplus_{\substack{P<R \leq S \\ \# \Delta_{P}^{R}=p}} I_{p_{w}} H_{U_{R} \cap\left|\Delta_{P}^{Q \vee R}\right|}^{j-1} \cong \bigoplus_{\substack{P<R \leq S \\ \# \Delta_{P}^{R}=p}} I_{p_{w}} H_{c\left(\left|\Delta_{R}^{Q \vee R}\right|\right)}^{j-1} .
$$

See Figure 26.

The term of (64) associated to $R>P$ vanishes by the truncation condition (61) at the vertex when $j-1-p>p_{w}(R)$, that is, when

$$
j>p_{w}(R)+\# \Delta_{P}^{R}
$$

Since $p$ is a middle perversity, it is easy to verify that

$$
p_{w}(P)=p_{w}(R)+\left\lfloor\left(\operatorname{dim} \mathfrak{n}_{P}^{R}+\# \Delta_{P}^{R}\right) / 2\right\rfloor-\ell^{R}(w)+\delta,
$$

where $\delta=0$ or 1 depending on the choice of $p$ and the parity of $\left(\operatorname{dim} \mathfrak{n}_{P}^{R}+\# \Delta_{P}^{R}\right)$ and $\left(\operatorname{dim} \mathfrak{n}_{P}+\# \Delta_{P}\right)$. However we know by Proposition 18.3 (applied to $w^{R}$ and

\footnotetext{
${ }^{4}$ We are using $p$ both as an index and a perversity; this should not cause confusion.
} 
the parabolic $P / N_{R}$ of $\left.L_{R}\right)$ that

$$
\ell^{R}(w) \leq\left(\operatorname{dim} \mathfrak{n}_{P}^{R}\right) / 2 .
$$

Thus the Mayer-Vietoris spectral sequence yields the vanishing of (63) for

$$
j>p_{w}(P)+\left\lceil \# \Delta_{P}^{R} / 2\right\rceil-\delta .
$$

Assume that $r(P)=3$. Then $\# \Delta_{P}^{R} \leq 2$ and thus (63) vanishes for $j>p_{w}(P)+1$ and even for $j=p_{w}(P)+1$ if $\delta=1$. Thus the Mayer-Vietoris spectral sequence argument yields a complete proof for the parabolic rank 3 case.

For $r(P) \geq 4$ the argument leading to (68) is not sufficient. It would be sufficient, however, if (67) were far enough away from equality. It could also be improved if we had more precise knowledge about $I_{p_{w}} H\left(\left|\Delta_{R}\right|\right)$, which suggests the utility of an inductive argument. On the other hand, the Fary spectral sequence explicitly calls out for an inductive argument. In fact, if we could apply (60) by induction, with $P$ replaced by $R, w$ by $w_{R}$, and $Q$ by $Q \vee R$, all the terms in (65) would vanish. Of course this is not possible (and would lead to too strong a result) since the hypotheses (i) and (ii) are not preserved under such an induction.

The approach in [61] is to replace (i) and (ii) by other hypotheses that are more suited to induction and use a combination of the two spectral sequences. Recall that (i) and (ii) were used as input to Proposition 18.3; more precisely they imply that for all $A_{P}$-weights $\alpha$ occurring in $\mathfrak{n}_{P}$,

$$
\begin{cases}\ell_{\alpha}(w) \geq\left(\operatorname{dim} \mathfrak{n}_{\alpha}\right) / 2 & \text { if } \alpha \text { occurs in } \mathfrak{n}_{P}^{Q} \\ \ell_{\alpha}(w) \leq\left(\operatorname{dim} \mathfrak{n}_{\alpha}\right) / 2 & \text { if } \alpha \text { occurs in } \mathfrak{n}_{P}^{S}\end{cases}
$$

Here $\operatorname{dim} \mathfrak{n}_{\alpha}$ is the number of $\mathbb{C}$-roots $\gamma$ such that $\left.\gamma\right|_{\mathfrak{a}_{P}}=\alpha$ and $\ell_{\alpha}(w)$ is the number of such roots which in addition satisfy $w^{-1} \gamma<0$. The conditions (69) are still not preserved by induction from $P$ to $R$. However we can prove $[\mathbf{6 1} \text {, Lemma 17.8 }]^{5}$ that there exists a parabolic $\mathbb{Q}$-subgroup $T \geq P$ (depending on $w$ ) with $r(T)=1$ or 2 and $r(Q \vee T), r(S \vee T) \leq 1$ such that for all $A_{P}$-weights $\alpha$ occurring in $\mathfrak{n}_{P}$,

$$
\begin{cases}\ell_{\alpha}(w) \geq\left(\operatorname{dim} \mathfrak{n}_{\alpha}\right) / 2 & \text { if } \alpha \text { occurs in } \mathfrak{n}_{T}^{Q \vee T}, \\ \ell_{\alpha}(w) \leq\left(\operatorname{dim} \mathfrak{n}_{\alpha}\right) / 2 & \text { if } \alpha \text { occurs in } \mathfrak{n}_{T}^{S \vee T} .\end{cases}
$$

We illustrate the difference between conditions (69) and (70) in Figure 27 (here $P \leq Q$ are as in Figure 22(b) and only one possible value of $T$ is considered). It is clear from the figure that significant information is gained in passing from (69) to (70) even though some information is lost as well. Clearly the conditions (70) are preserved by induction from $P$ to $R$ provided $R \leq T$.

The proof of (62a)-(62c), and hence $I_{p_{w}} H_{c\left(\left|\Delta_{P}^{Q}\right|\right)}=0$, in [61, Theorem 17.9] uses the existence of $T$ satisfying (70) (plus one additional hypothesis). The proof of (62a) is separated into two parts. The vanishing of $I_{p_{w}} H_{\left|\Delta_{P}^{Q \vee V T}\right| \backslash\left|\Delta_{P}^{Q}\right|}^{j-1}$, is proved using the Fary spectral sequence and induction from $P$ to $R \leq T$. The other part,

\footnotetext{
${ }^{5}$ This is where the condition on the $\mathbb{Q}$-root system in Theorem 18.1 comes into play. Recall that a quasi-minuscule representation is one in which the non-zero weights form a single Weyl group orbit. The root systems of type $A_{n}, B_{n}, C_{n}$, and $G_{2}$ are precisely the irreducible root systems such that the corresponding simply connected $\mathbb{Q}$-split group has a quasi-minuscule representation whose weights under the usual ordering are totally ordered; thus our hypothesis allows us to express the $\mathbb{Q}$-roots of $G$ as differences of weights $\varepsilon_{i}-\varepsilon_{j}$ analogously to the usual expression of roots in $\mathrm{GL}_{n}$. We expect that a further study of quasi-minuscule representations will allow us to treat the cases $D_{n}, E_{n}$, and $F_{4}$, although we may have to allow $r(T)=3$ or 4 .
} 


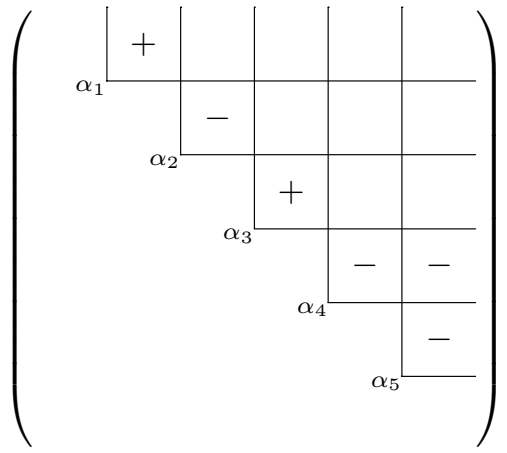

(a) $-=\mathfrak{n}_{P}^{Q}$

$+=\mathfrak{n}_{P}^{S}$

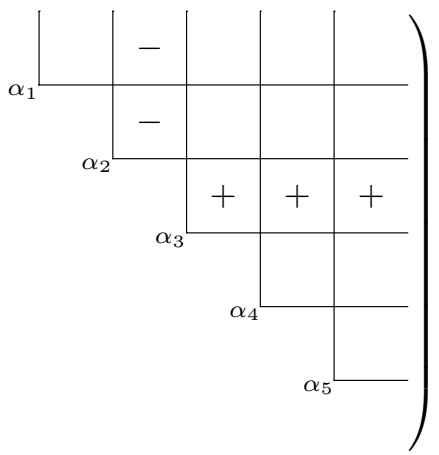

(a) $\quad-=\mathfrak{n}_{T}^{Q \vee T}$ $+=\mathfrak{n}_{T}^{S \vee T}$

Figure 27. An example of $\mathfrak{n}_{P}^{Q}$ versus $\mathfrak{n}_{T}^{Q \vee T}$ and $\mathfrak{n}_{P}^{S}$ versus $\mathfrak{n}_{T}^{S \vee T}$ in $\mathrm{GL}_{n}(\mathbb{R})$. Here $\Delta_{P}=\left\{\alpha_{1}, \ldots, \alpha_{5}\right\}, \Delta_{P}^{Q}=\left\{\alpha_{2}, \alpha_{4}, \alpha_{5}\right\}$, and $\Delta_{P}^{T}=$ $\left\{\alpha_{1}, \alpha_{4}, \alpha_{5}\right\}$.

the vanishing of $I_{p_{w}} H^{j-1}\left(\left|\Delta_{P}\right| \backslash\left|\Delta_{P}^{Q \vee T}\right|\right)$, is proved with the Mayer-Vietoris spectral sequence (note that $\# \Delta_{P}^{R} \leq 1$ in this case).

\section{Functoriality of micro-support}

It is important to understand how the micro-support of an $\mathcal{L}$-module $\mathcal{M}$ changes when the various functors $k_{*}, k^{*}$, and $k^{!}$are applied to $\mathcal{M}$. An example of this for the case of a direct image functor has already been illustrated in $\$ 13.2$ with the calculation of $\operatorname{SS}\left(i_{G *} E\right)$. It remains to consider inverse image functors. Specifically one would like understand how $\operatorname{SS}\left(k^{*} \mathcal{M}\right)$, for example, is derived from $\operatorname{SS}(\mathcal{M})$. Furthermore, in view of Theorem 14.1, it is important also to estimate how $c\left(k^{*} \mathcal{M}\right)$ and $d\left(k^{*} \mathcal{M}\right)$ relate to the corresponding quantities for $\mathcal{M}$. A number of general theorems of this sort are found in [61]; here we will instead simply illustrate these ideas by sketching the proof of the following result needed for Theorem 11.1 and illustrated in Figure 28. The complete proof is found in [61, Corollary 26.2].

Theorem 19.1. Let $\mathcal{M}$ be an $\mathcal{L}$-module on $\widehat{X}$ with $\operatorname{SS}_{\text {ess }}(\mathcal{M})=\{E\}$ and $c(E ; \mathcal{M})=d(E ; \mathcal{M})=0$. Let $x \in X_{R, h}$ be a point on a stratum of a real equalrank Satake compactification $X^{*}$. Let $\pi: \widehat{X} \rightarrow X^{*}$ be Zucker's projection and let $k: \pi^{-1}(x) \cong \widehat{X}_{R, \ell} \hookrightarrow \widehat{X}$ denote the inclusion. Then

$$
d\left(k^{*} \mathcal{M}\right) \leq \frac{1}{2} \operatorname{codim} X_{R, h}-\# \Delta_{R} \quad \text { and } \quad c(k ! \mathcal{M}) \geq \frac{1}{2} \operatorname{codim} X_{R, h}+\# \Delta_{R} .
$$

We will make several simplifying assumptions along the way in order to highlight the basic idea. To begin with though, we do not make any assumption about $\operatorname{SS}(\mathcal{M})$ nor about the equal-rank nature of the boundary components of $X^{*}$; these will be added later to emphasize where they are needed.

19.1. Consider the triple of subsets,

$$
\{x\} \subseteq X_{R, h} \subseteq U_{R, h} \subseteq X^{*},
$$




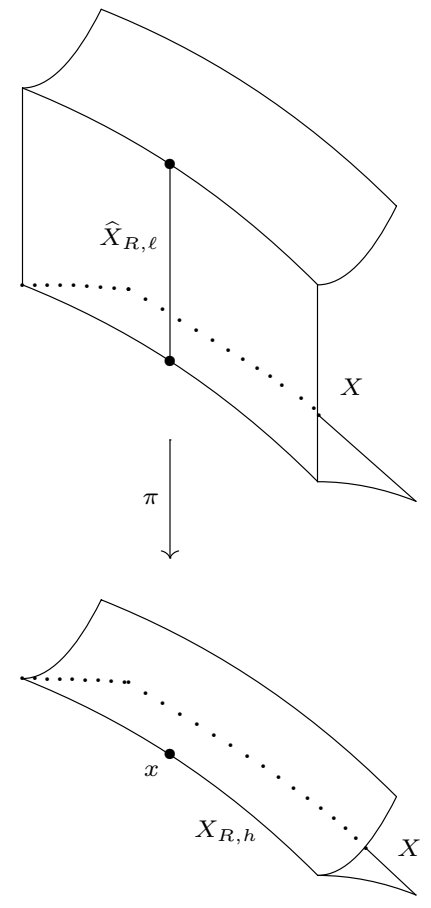

Figure 28. The projection $\pi: \widehat{X} \rightarrow X^{*}$ over a point $x \in X_{R, h}$ (adapted from $[\mathbf{6 2}]$ )

where

$$
U_{R, h} \equiv \coprod_{\operatorname{cl}\left(X_{S, h)} \supseteq X_{R, h}\right.} X_{S, h}
$$

is the open star neighborhood of $X_{R, h}$. The inclusion $k$ may thus be factored into the composition of three inclusions,

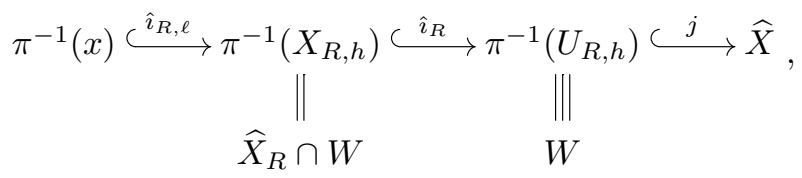

and we will examine (in reverse order) how each affects micro-support. For simplicity we will only consider $k^{*}$; the functor $k^{!}$is treated similarly.

19.2. An open embedding. The map

$$
j: W=\coprod_{\substack{P \leq R \\ P^{\dagger}=R}} \coprod_{\substack{Q \geq P \\ Q \cap R=P}} X_{Q} \longleftrightarrow \widehat{X}
$$

is an embedding of an open admissible subspace. The effect on micro-support is simple [61, Proposition 22.2]:

$$
\mathrm{SS}\left(j^{*} \mathcal{M}\right)=\operatorname{SS}(\mathcal{M}) \cap\left(\coprod_{Q \in \mathcal{P}(W)} \mathfrak{I r r}\left(L_{Q}\right)\right)
$$




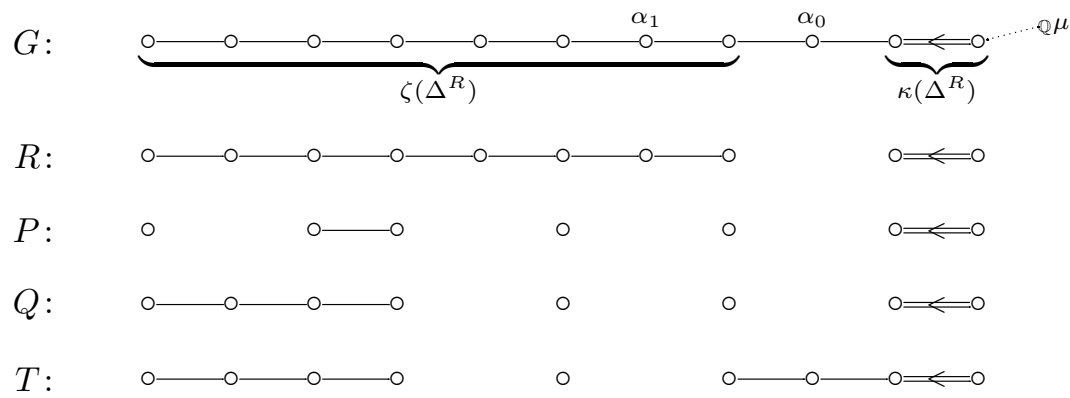

Figure 29. An example of the Dynkin diagrams for $G$ and the Levi quotients of various parabolic $\mathbb{Q}$-subgroups considered in the text. (Here $G=\operatorname{Sp}_{22}(\mathbb{R})$ and $X^{*}$ is the Baily-Borel-Satake compactification.)

and for $V \in \operatorname{SS}\left(j^{*} \mathcal{M}\right), c\left(V ; j^{*} \mathcal{M}\right)=c(V ; \mathcal{M})$ and $d\left(V ; j^{*} \mathcal{M}\right)=(V ; \mathcal{M})$.

19.3. The inclusion of the closure of a stratum. The next map

$$
\hat{\imath}_{R}: \widehat{X}_{R} \cap W=\coprod_{\substack{P \leq R \\ P^{\dagger}=R}} X_{P} \longleftrightarrow W
$$

is the main issue; it is the embedding of a (relatively) closed stratum. To treat it we outline a simplified version of the arguments of [61, Propositions 22.6 and 23.3]. Set $\mathcal{M}_{1}=j^{*} \mathcal{M}$ whose micro-support we understand by (71). Let $P \in \mathcal{P}\left(\widehat{X}_{R} \cap W\right)$. If an $L_{P}$-module $V$ belongs to $\operatorname{SS}\left(\hat{\imath}_{R}^{*} \mathcal{M}_{1}\right)$, then $\left.V\right|_{M_{P}}$ is conjugate self-contragredient and

$$
H^{j}\left(i_{P}^{*} \hat{\imath}_{Q}^{!}\left(\hat{\imath}_{R}^{*} \mathcal{M}_{1}\right)\right)_{V} \neq 0
$$

for some $Q \leq R$ such that $Q_{V}^{R} \leq Q \leq Q_{V}^{\prime R}$ and some $j$. Note that $\hat{\imath}_{Q}^{!} \hat{\imath}_{R}^{*} \mathcal{M}_{1}=\hat{\imath}_{T}^{!} \mathcal{M}_{1}$ where $T \geq Q$ is the unique parabolic $\mathbb{Q}$-subgroup complementary to $R$ relative to $Q$, that is, such that $\Delta_{Q}^{T}=\Delta_{Q} \backslash \Delta_{Q}^{R}$. (See Figure 29 for an example of $P, Q, R$, and $T$.) Thus we have

$$
H^{j}\left(i_{P}^{*} \hat{\imath}_{T}^{!} \mathcal{M}_{1}\right)_{V} \neq 0
$$

We will show that if $V$ is not already in $\operatorname{SS}\left(\mathcal{M}_{1}\right)$, then it is explicitly related to an element $\tilde{V} \in \operatorname{SS}\left(\hat{\imath}_{\tilde{R}}^{*} \mathcal{M}_{1}\right)$, where $X_{\tilde{R}, h}$ is a larger stratum in $U_{R, h}$. A repetition of the argument will then lead us to an element in $\operatorname{SS}\left(\mathcal{M}_{1}\right)$.

For simplicity now assume:

(i) The Dynkin diagram of the $\mathbb{Q}$-root system for $G$ is linear.

(ii) The highest $\mathbb{Q}$-weight ${ }_{\mathbb{Q}} \mu$ of the representation defining the Satake compactification is orthogonal to all simple $\mathbb{Q}$-roots except one at the end of the Dynkin diagram.

(iii) All rational boundary components are equal-rank.

These conditions are satisfied for practically all the real equal-rank Satake compactifications (including the Baily-Borel-Satake compactification) considered in Theorem 11.1, and in particular for the example illustrated in Figure 29. Assumptions (i) and (ii) are simply for ease of exposition; they ensure that 
- All saturated parabolic $\mathbb{Q}$-subgroups $R$ are maximal.

Assumption (iii) will be needed to ensure that $\tilde{V}$ below is actually conjugate selfcontragredient.

Since $R$ is maximal by the above assumptions, write $\Delta_{P}^{R}=\Delta_{P} \backslash\left\{\alpha_{0}\right\}$ and let $\tilde{P}>P$ have $\Delta_{P}^{\tilde{P}}=\left\{\alpha_{0}\right\}$. We need to understand how $T$ relates to $Q_{V}$ and $Q_{V}^{\prime}$. By definition, $T=Q \vee \tilde{P}$, that is, $\Delta_{P}^{T}=\Delta_{P}^{Q} \cup\left\{\alpha_{0}\right\}$. For $Q_{V}$ and $Q_{V}^{\prime}$ there are three cases:

(a) $\left(\xi_{V}+\rho, \alpha_{0}\right)<0$ and thus $Q_{V}=Q_{V}^{R} \vee \tilde{P}$ and $Q_{V}^{\prime}=Q_{V}^{\prime R} \vee \tilde{P}$.

(b) $\left(\xi_{V}+\rho, \alpha_{0}\right)=0$ and thus $Q_{V}=Q_{V}^{R}$ and $Q_{V}^{\prime}=Q_{V}^{\prime R} \vee \tilde{P}$.

(b) $\left(\xi_{V}+\rho, \alpha_{0}\right)>0$ and thus $Q_{V}=Q_{V}^{R}$ and $Q_{V}^{\prime}=Q_{V}^{\prime R}$.

In cases (a) and (b), $Q_{V} \leq T \leq Q_{V}^{\prime}$ and thus equation (72) implies that $V \in$ $\mathrm{SS}\left(\mathcal{M}_{1}\right)$. In case (c) on the other hand, $T \not \leq Q_{V}^{\prime}$.

We investigate case (c) further. Consider the long exact sequence of the triple $\left(U, U \backslash\left(U \cap \widehat{X}_{Q}\right), U \backslash\left(U \cap \widehat{X}_{T}\right)\right)$, where $U$ is a small neighborhood of a point of $X_{P}$. The $V$-isotypical part of this sequence is

$$
\cdots \longrightarrow H^{j}\left(i_{P}^{*} \hat{\imath}_{Q}^{!} \mathcal{M}_{1}\right)_{V} \longrightarrow H^{j}\left(i_{P}^{*} \hat{\imath}_{T}^{!} \mathcal{M}_{1}\right)_{V} \longrightarrow H^{j}\left(i_{P}^{*} i_{\tilde{P} *}\left(i_{\tilde{P}}^{*} \hat{l}_{T}^{!} \mathcal{M}_{1}\right)\right)_{V} \longrightarrow \cdots
$$

By (72), the middle term is nonzero for some $j$, so either the first or last term must also be nonzero. If the first term is nonzero, again $V \in \operatorname{SS}\left(\mathcal{M}_{1}\right)$ since $Q_{V} \leq Q \leq$ $Q_{V}^{\prime}$. On the other hand, it is easy to see that the last term can be rewritten as $H^{\ell}\left(\mathfrak{n}_{P}^{\tilde{P}} ; H^{j-\ell}\left(i_{\tilde{P}}^{*} \hat{l}_{T}^{!} \mathcal{M}_{1}\right)\right)_{V}$, so if it is nonzero we must have

$$
H^{j-\ell(w)}\left(i_{\tilde{P}}^{*} \hat{\imath}_{T}^{!} \mathcal{M}_{1}\right)_{\tilde{V}} \neq 0
$$

for some irreducible $L_{\tilde{P}}$-module $\tilde{V}$ such that $V=H^{\ell(w)}\left(\mathfrak{n}_{P}^{\tilde{P}} ; \tilde{V}\right)_{w}$ for some $w \in$ $W_{P}^{\tilde{P}}$. It is not difficult to check $\left[\mathbf{6 1}\right.$, Lemma 23.2] that $\left.\tilde{V}\right|_{M_{\tilde{P}}}$ is conjugate selfcontragredient from (iii) and the fact that any representation of an equal-rank group is conjugate self-contragredient.

By assumptions (i) and (ii), the roots in $\Delta_{P}$ have a linear ordering induced from that of $\Delta$ and $\alpha_{0}$ will occur at one end; this means that $\alpha_{0}$ is orthogonal to all $\alpha \in \Delta_{P} \backslash \Delta_{P}^{\tilde{P}}$ except for one, say $\alpha_{1}$. Set $\tilde{\alpha}=\left.\alpha\right|_{A_{\tilde{P}}}$ for $\alpha \in \Delta_{P} \backslash \Delta_{P}^{\tilde{P}}$. One can show [61, Lemmas 22.5 and 23.1] that

$$
\left(\xi_{\tilde{V}}+\rho, \tilde{\alpha}\right) \begin{cases}=\left(\xi_{V}+\rho, \alpha\right) & \text { for } \alpha \neq \alpha_{1}, \\ >\left(\xi_{V}+\rho, \alpha_{1}\right) & \text { for } \alpha=\alpha_{1}\end{cases}
$$

(the inequality is since we are in case (c)). Thus if $\alpha_{1} \notin \Delta_{P}^{Q}$ (as in Figure 29), then $Q_{\tilde{V}} \leq T \leq Q_{\tilde{V}}^{\prime}$ and hence (73) implies $\tilde{V} \in \operatorname{SS}\left(\mathcal{M}_{1}\right)$.

If $\alpha_{1} \in \Delta_{P}^{Q}$, we want to apply to (73) the reverse of the reasoning that led to (72). Set $\tilde{R}$ to be the maximal $\mathbb{Q}$-parabolic subgroup with $\Delta_{P}^{\tilde{R}}=\Delta_{P} \backslash\left\{\alpha_{1}\right\}$ and set $\tilde{Q} \equiv \tilde{R} \cap T$ (see Figure 30). Then $\hat{\imath}_{T}^{!} \mathcal{M}_{1}=\hat{\imath}_{\tilde{Q}}^{!} \hat{\imath}_{\tilde{R}}^{*} \mathcal{M}_{1}$ and equation (74) implies that

$$
Q_{\tilde{V}}^{\tilde{R}} \leq \tilde{Q} \leq Q_{\tilde{V}}^{\prime \tilde{R}}
$$

Thus we have

$$
H^{j-\ell(w)}\left(i_{\tilde{P}}^{*} \hat{l}_{\tilde{Q}}^{!}\left(\hat{\imath}_{\tilde{R}}^{*} \mathcal{M}_{1}\right)\right)_{\tilde{V}} \neq 0
$$

and hence $\tilde{V} \in \operatorname{SS}\left(\hat{\imath}_{\tilde{R}}^{*} \mathcal{M}_{1}\right)$. In this case we may repeat the argument with $V$ replaced by $\tilde{V}$ to eventually obtain an element of $\operatorname{SS}\left(\mathcal{M}_{1}\right)$. 


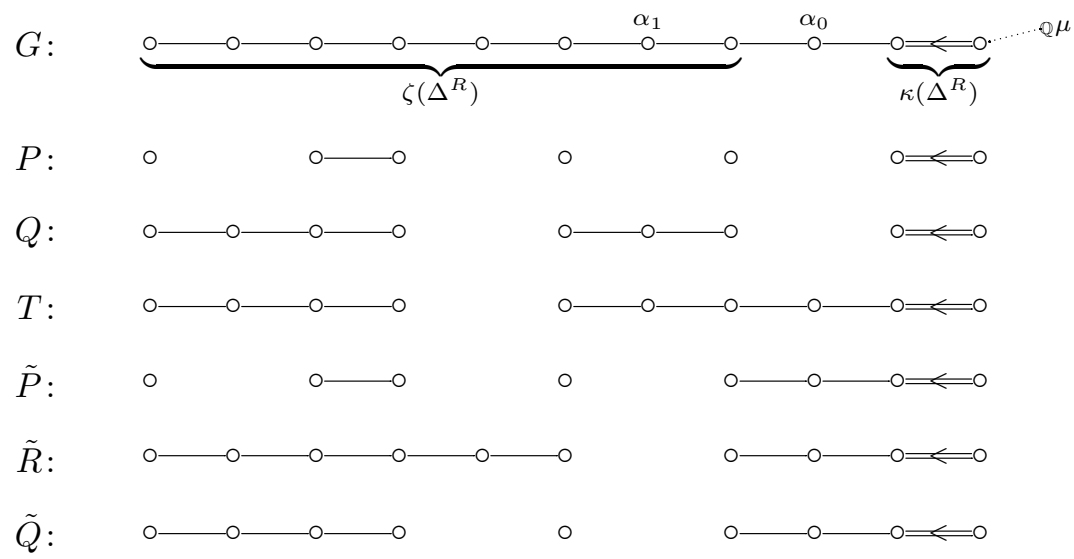

Figure 30. The case $\alpha_{1} \in \Delta_{P}^{Q}$

In conclusion, we see that for every $V \in \operatorname{SS}\left(\hat{\imath}_{R}^{*} \mathcal{M}_{1}\right)$, either

(i) $V \in \mathrm{SS}\left(\mathcal{M}_{1}\right)$, or

(ii) $V=H^{\ell(w)}\left(\mathfrak{n}_{P}^{\tilde{P}} ; \tilde{V}\right)_{w}$ for an $L_{\tilde{P}}$-module $\tilde{V} \in \operatorname{SS}\left(\mathcal{M}_{1}\right)$ satisfying

$$
\begin{gathered}
\tilde{P} \cap R=P, \\
\left(\xi_{V}+\rho, \alpha\right)>0 \text { for all } \alpha \in \Delta_{P}^{\tilde{P}}, \text { and } \\
d\left(V ; \hat{\imath}_{R}^{*} \mathcal{M}_{1}\right) \leq d\left(\tilde{V} ; \mathcal{M}_{1}\right)+\ell(w),
\end{gathered}
$$

or

(iii) $V$ is obtained from an element of $\operatorname{SS}\left(\mathcal{M}_{1}\right)$ by a sequence of steps similar to (ii).

19.4. The inclusion of a fiber. The set $\widehat{X}_{R} \cap W=\pi^{-1}\left(X_{R, h}\right)$ is a flat bundle over $X_{R, h}$ with typical fiber $\widehat{X}_{R, \ell}$. Our final map

$$
\hat{\imath}_{R, \ell}: \pi^{-1}(x) \cong \widehat{X}_{R, \ell} \longleftrightarrow \widehat{X}_{R} \cap W
$$

is the inclusion of such a fiber. Thus $\widehat{X}_{R, \ell}$ is not an admissible subspace of $\widehat{X}_{R} \cap W$ and the functors $\hat{\imath}_{R, \ell}^{*}$ and $\hat{\imath}_{R, \ell}^{!}$are defined differently from those in $\S 12.3$.

Instead, note that the spaces $\widehat{X}_{R, \ell}$ and $\widehat{X}_{R} \cap W$ have strata indexed by the same set: $P \in \mathcal{P}$ such that $P \leq R$ with $P^{\dagger}=R$. For such $P$, the corresponding stratum of $\widehat{X}_{R, \ell}$ is $X_{P, \ell}$ while the corresponding stratum of $\widehat{X}_{R} \cap W$ is $X_{P}$. Let $\mathcal{M}_{2} \equiv \hat{\imath}_{R}^{*} j^{*} \mathcal{M}=\left(E ., f\right.$.) be an $\mathcal{L}$-module on $\widehat{X}_{R} \cap W$ (whose micro-support we understand by the preceding two subsections). The $\mathcal{L}$-module $\hat{\imath}_{R, \ell}^{*} \mathcal{M}_{2}=\left(E_{.}^{\prime}, f_{.}^{\prime}\right)$ on $\widehat{X}_{R, \ell}$ is defined $[\mathbf{6 1}, \S 3.5]$ by

$$
\begin{aligned}
E_{P_{\ell}}^{\prime} & \equiv \operatorname{Res}_{L_{P, \ell}}^{L_{P}} E_{P}, \\
f_{P_{\ell} Q_{\ell}}^{\prime} & \equiv \operatorname{Res}_{L_{P, \ell}}^{L_{P}} f_{P Q} ;
\end{aligned}
$$

the $\mathcal{L}$-module $\hat{\imath}_{R, \ell}^{!} \mathcal{M}_{2}$ is defined similarly but with a degree shift of $-\operatorname{dim} D_{R, h}$. 
The effect of $\hat{\imath}_{R, \ell}^{*}$ (and likewise $\hat{\imath}_{R, \ell}^{\prime}$ ) on micro-support is simple [61, Proposition 2.8]:

$$
\operatorname{SS}\left(\hat{\imath}_{R, \ell}^{*} \mathcal{M}_{2}\right)=\left\{\operatorname{Res}_{L_{P, \ell}}^{L_{P}} V \mid V \in \operatorname{SS}\left(\mathcal{M}_{2}\right)\right\} .
$$

(This is an abuse of notation since $\operatorname{Res}_{L_{P, \ell}}^{L_{P}} V$ is not actually an irreducible $L_{P, \ell^{-}}$ module, merely isotypical. In the above formula we mean the unique irreducible $L_{P, \ell}$-module that occurs in $\operatorname{Res}_{L_{P, \ell}}^{L_{P}} V$.)

19.5. Sketch of the proof of Theorem 19.1. Since $k^{*} \mathcal{M}=\hat{\imath}_{R, \ell}^{*} \hat{\imath}_{R}^{*} j^{*} \mathcal{M}$ and we assume that $\mathrm{SS}_{\mathrm{ess}}(\mathcal{M})=\{E\}$ with $c(E ; \mathcal{M})=d(E ; \mathcal{M})=0$, Theorem 19.1 may be deduced from the 3 preceding subsections. The only issue is to estimate $\ell(w)$ which is accomplished by the following proposition. This is the point at which we need to assume that all real (as opposed to rational) boundary components are equal-rank.

Proposition 19.2. Let ${ }_{\mathbb{R}} D^{*}$ be a real equal-rank Satake compactification. Let $P$ be a parabolic $\mathbb{Q}$-subgroup and let $w \in W_{P}$. Let $V=H^{\ell(w)}\left(\mathfrak{n}_{P} ; E\right)_{w}$ be the corresponding irreducible $L_{P}$-module and assume that $\left(\left.V\right|_{M_{P}}\right)^{*} \cong \overline{\left.V\right|_{M_{P}}}$.

(i) If $\left(\xi_{V}+\rho, \alpha\right) \leq 0$ for all $\alpha \in \Delta_{P}$, then

$$
\ell(w) \geq\left(\operatorname{dim} \mathfrak{n}_{P}+\# \Delta_{P}+\operatorname{dim} D_{P, \ell}(V)\right) / 2 .
$$

(ii) If $\left(\xi_{V}+\rho, \alpha\right) \geq 0$ for all $\alpha \in \Delta_{P}$, then

$$
\ell(w) \leq\left(\operatorname{dim} \mathfrak{n}_{P}-\# \Delta_{P}-\operatorname{dim} D_{P, \ell}(V)\right) / 2 .
$$

The proposition follows from Lemma 15.2 and the estimate [61, Lemma 25.2]

$$
\operatorname{dim} \mathfrak{n}_{P}(V) \geq \# \Delta_{P}+\operatorname{dim} D_{P, \ell}(V) .
$$

A similar estimate was proved in $[\mathbf{1 1}, \S 5.5]$ which only requires that $D$ and $D_{R, h}$ are equal-rank and that a certain condition (B) holds which has to be verified case-by-case (it is satisfied for most real equal-rank Satake compactifications.) The proof of the estimate in [61] is free from case-by-case analysis but strongly uses the assumption that all real boundary components are equal-rank.

\section{Proof of the Rapoport/Goresky-MacPherson conjecture}

To prove Theorem 11.1 we need to show that $\pi_{*} \mathcal{I}_{p} \mathcal{C}(\widehat{X} ; \mathbb{E}) \cong \mathcal{I}_{p} \mathcal{C}\left(X^{*} ; \mathbb{E}\right)$. This is done by verifying that $\pi_{*} \mathcal{I}_{p} \mathcal{C}(\widehat{X} ; \mathbb{E})$ satisfies the local characterization of intersection cohomology on $X^{*}$, conditions (i)-(iii) from $\S 4.4$. Condition (i) is obvious. Let $i_{x}:\{x\} \hookrightarrow X^{*}$ denote the inclusion of a point $x \in X^{*}$. The local vanishing condition (ii) amounts to

$$
H^{j}\left(i_{x}^{*} \pi_{*} \mathcal{I}_{p} \mathcal{C}(\widehat{X} ; \mathbb{E})\right)=0 \quad \text { for } x \in X_{R, h}, j \geq \frac{1}{2} \operatorname{codim} X_{R, h}
$$

since $p(k)=k / 2-1$ for either middle perversity when $k$ is even. Given the other conditions and constructibility, the attaching condition (iii) is equivalent [15, V.4], [33] to a local covanishing condition:

$$
H^{j}\left(i_{x}^{!} \pi_{*} \mathcal{I}_{p} \mathcal{C}(\widehat{X} ; \mathbb{E})\right)=0 \quad \text { for } x \in X_{R, h}, j \leq \frac{1}{2} \operatorname{codim} X_{R, h} .
$$

Let $k: \pi^{-1}(x) \hookrightarrow \widehat{X}$ be the inclusion. We may re-express

$$
H^{j}\left(i_{x}^{*} \pi_{*} \mathcal{I}_{p} \mathcal{C}(\widehat{X} ; \mathbb{E})\right) \cong H^{j}\left(\pi^{-1}(x) ; k^{*} \mathcal{I}_{p} \mathcal{C}(\widehat{X} ; \mathbb{E})\right) ;
$$


since $\mathcal{I}_{p} \mathcal{C}(\widehat{X} ; \mathbb{E})=\mathcal{S}\left(\mathcal{I}_{p} \mathcal{C}(\widehat{X} ; E)\right)$, this is isomorphic to the $\mathcal{L}$-module cohomology $H^{j}\left(\widehat{X}_{R, \ell} ; k^{*} \mathcal{I}_{p} \mathcal{C}(\widehat{X} ; E)\right)$. Thus we can use Theorem 14.1 to see this vanishes for $j>d\left(k^{*} \mathcal{I}_{p} \mathcal{C}(\widehat{X} ; E)\right)$. By Theorem 18.1, we know that $\operatorname{SS}_{\text {ess }}\left(\mathcal{I}_{p} \mathcal{C}(\widehat{X} ; E)\right)=\{E\}$ and hence $d\left(k^{*} \mathcal{I}_{p} \mathcal{C}(\widehat{X} ; E)\right) \leq \frac{1}{2} \operatorname{codim} X_{R, h}-1$ by Theorem 19.1. This establishes (76); an analogous argument treats $(77)$.

\section{A generalization of Goresky-Harder-MacPherson's theorem}

The weighted cohomology $\mathcal{L}$-module $\mathcal{W}^{\eta} \mathcal{C}(\widehat{X} ; E)$ may be constructed $[\mathbf{6 1}, \S 6]$ by a weight truncation functor similar to the degree truncation functor that is used for $\mathcal{I}_{p} \mathcal{C}(\widehat{X} ; E)$. The following analogue of Theorem 18.1 is proved in [61, Theorem 16.3]. Note that there is no assumption on the $\mathbb{Q}$-root system. Unlike Theorem 18.1, this theorem is not difficult to prove; this is because there is an explicit non-inductive formula for the local weighted cohomology.

Theorem 21.1. Let $E$ be an irreducible $G$-module and let $\eta=\mu$ or $\nu$ be a middle-weight profile. If $\left(\left.E\right|_{o_{G}}\right)^{*} \cong \overline{\left.E\right|_{o_{G}}}$, then $\operatorname{SS}_{\mathrm{ess}}\left(\mathcal{W}^{\eta} \mathcal{C}(\widehat{X} ; E)\right)=\{E\}$.

Consequently, the argument in the preceding section also proves the following generalization of Goresky, Harder, and MacPherson's result [30], Theorem 10.1:

THeOREM 21.2. Let $X^{*}$ be a real equal-rank Satake compactification, let $p$ be a middle-perversity, and let $\eta$ be a middle-weight profile. There is a natural quasiisomorphism $\mathcal{I}_{p} \mathcal{C}\left(X^{*} ; \mathbb{E}\right) \cong \pi_{*} \mathcal{W}^{\eta} \mathcal{C}(\widehat{X} ; \mathbb{E})$ and hence an isomorphism $I_{p} H\left(X^{*} ; \mathbb{E}\right) \cong$ $W^{\eta} H(\widehat{X} ; \mathbb{E})$.

\section{References}

1. J. Arthur, A trace formula for reductive groups. I. Terms associated to classes in $G(\mathbf{Q})$, Duke Math. J. 45 (1978), 911-953.

2. — The $L^{2}$ Lefschetz numbers of Hecke operators, Inv. Math. 97 (1989), 257-290.

3. A. Ash, D. Mumford, M. Rapoport, and Y. Tai, Smooth compactification of locally symmetric varieties, Math Sci Press, Brookline, 1975.

4. W. Baily and A. Borel, Compactification of arithmetic quotients of bounded symmetric domains, Ann. of Math. 84 (1966), 442-528.

5. G. Barthel, J.-P. Brasselet, K.-H. Fieseler, and L. Kaup, Equivariant intersection cohomology of toric varieties, Algebraic geometry: Hirzebruch 70 (Warsaw, 1998), Contemp. Math., vol. 241, Amer. Math. Soc., Providence, RI, 1999, pp. 45-68.

6. _ Combinatorial intersection cohomology for fans, Tohoku Math. J. (2) 54 (2002), no. $1,1-41$.

7. A. Beilinson, J. Bernstein, and P. Deligne, Faisceaux pervers, Analyse et topologie sur les espaces singuliers (I), CIRM, 6-10 juillet 1981, Astérisque, vol. 100, 1982.

8. A. Borel, Ensembles fondamentaux pour les groupes arithmétiques, Colloque sur la Théorie des Groupes Algébriques, Tenu à Bruxelles du 5 au 7 juin 1962, Centre Belge de Recherches Mathématiques, 1962, pp. 23-40.

9. - Introduction aux groupes arithmétiques, Hermann, Paris, 1969.

10.,$L^{2}$-cohomology and intersection cohomology of certain arithmetic varieties, Emmy Noether in Bryn Mawr (Bryn Mawr, Pa., 1982), Springer, New York, 1983, pp. 119-131.

11. _ A vanishing theorem in relative Lie algebra cohomology, Algebraic Groups: Utrecht 1986 (Berlin) (A. M. Cohen, W. H. Hesselink, W. L. J. van der Kallen, and J. R. Strooker, eds.), Lecture Notes in Mathematics, vol. 1271, Springer-Verlag, 1987, pp. 1-16.

12. Linear algebraic groups, Springer-Verlag, Berlin, 1991.

13. A. Borel and W. Casselman, $L^{2}$-cohomology of locally symmetric manifolds of finite volume, Duke Math. J. 50 (1983), 625-647.

14. Cohomologie d'intersection et $L^{2}$-cohomologie de variétés arithmétiques de rang rationnel 2, C. R. Acad. Sci. Paris Sér. I Math. 301 (1985), no. 7, 369-373. 
15. A. Borel et al., Intersection cohomology, Birkhäuser, Boston, 1984.

16. A. Borel and J.-P. Serre, Corners and arithmetic groups, Comment. Math. Helv. 48 (1973), 436-491.

17. A. Borel and J. Tits, Groupes réductifs, I.H.E.S. Publ. Math. 27 (1965), 55-151.

18. A. Borel and N. Wallach, Continuous cohomology, discrete subgroups, and representation of reductive groups, Ann. of Math. Stud., vol. 94, Princeton University Press, Princeton, 1980.

19. T. Braden, Hyperbolic localization of intersection cohomology, preprint, arXiv:math.AG/0202251, 9 pages, to appear in Transform. Groups, 2002.

20. Kـ Koszul duality for toric varieties, preprint, 2003.

21. T. Braden and R. MacPherson, From moment graphs to intersection cohomology, Math. Ann. 321 (2001), no. 3, 533-551.

22. P. Bressler and V. A. Lunts, Toric varieties and minimal complexes, preprint, arXiv:alg-geom/9712007, 11 pages, 1997.

23. I Intersection cohomology on nonrational polytopes, Compositio Math. 135 (2003), no. 3, 245-278.

24. W. Casselman, $L^{2}$-cohomology for groups of real rank one, Representation Theory of Reductive Groups (P. Trombi, ed.), Birkhäuser, Boston, 1983, pp. 69-84.

25. - Geometric rationality of Satake compactifications, Algebraic groups and Lie groups, Austral. Math. Soc. Lect. Ser., 9, Cambridge Univ. Press, Cambridge, 1997, pp. 81-103.

26. J. Cheeger, On the Hodge theory of Riemannian pseudomanifolds, Geometry of the Laplace Operator (Providence), Proc. Symposia Pure Math., vol. 36, Amer. Math. Soc., 1980.

27. J. Cheeger, M. Goresky, and R. MacPherson, $L^{2}$-cohomology and intersection homology of singular algebraic varieties, Seminar on Differential Geometry, Ann. of Math. Stud., vol. 102, Princeton Univ. Press, Princeton, N.J., 1982, pp. 303-340.

28. G. de Rham, Variétés différentiables, Hermann, Paris, 1973.

29. J. Franke, Harmonic analysis in weighted $L_{2}$-spaces, Ann. Sci. École Norm. Sup. (4) 31 (1998), 181-279.

30. M. Goresky, G. Harder, and R. MacPherson, Weighted cohomology, Invent. Math. 116 (1994), 139-213.

31. M. Goresky, R. Kottwitz, and R. MacPherson, Discrete series characters and the Lefschetz formula for Hecke operators, Duke Math. J. 89 (1997), 477-554.

32. M. Goresky and R. MacPherson, Intersection homology theory, Topology 19 (1980), no. 2, $135-162$.

33. Intersection homology II, Invent. Math. 72 (1983), 77-129.

34. Weighted cohomology of Satake compactifications, Centre de recherches mathématiques, preprint \#1593, 1988.

35. _ Local contribution to the Lefschetz fixed point formula, Invent. Math. 111 (1993), no. $1,1-33$.

36. The topological trace formula, preprint, arXiv:math.RT/0105038, 74 pages, 2001.

37. N. Habegger and L. Saper, Intersection cohomology of cs-spaces and Zeeman's filtration, Invent. Math. 105 (1991), no. 2, 247-272.

38. Harish-Chandra, Representations of semisimple Lie groups VI: Integrable and squareintegrable representations, Amer. J. Math. 78 (1956), no. 3, 564-628.

39. F. Hirzebruch, Hilbert modular surfaces, Enseignement Math. (2) 19 (1973), 183-281.

40. J. Humphreys, Linear algebraic groups, Springer-Verlag, New York, 1975, Graduate Texts in Mathematics, No. 21.

41. M. Kashiwara, The Riemann-Hilbert problem for holonomic systems, Publ. Res. Inst. Math. Sci. 20 (1984), no. 2, 319-365.

42. M. Kashiwara and P. Schapira, Sheaves on manifolds, Springer-Verlag, Berlin, 1990.

43. H. King, Topological invariance of intersection homology without sheaves, Topology Appl. 20 (1985), no. 2, 149-160.

44. B. Kostant, On the conjugacy of real Cartan subalgebras. I., Proc. Nat. Acad. Sci. U. S. A. 41 (1955), 967-970.

45. L Lie algebra cohomology and the generalized Borel-Weil theorem, Ann. of Math. 74 (1961), 329-387.

46. S. Kumaresan, On the canonical $k$-types in the irreducible unitary $g$-modules with nonzero relative cohomology, Invent. Math. 59 (1980), no. 1, 1-11. 
47. R. P. Langlands and D. Ramakrishnan (eds.), The zeta functions of Picard modular surfaces, Les Publications CRM, Montréal, 1992.

48. J.-S. Li, A vanishing result for the cohomology of arithmetic groups, Talk at the Conference "Representation Theory of Lie Groups (July 2002 - January 2003)", National University of Singapore, August 2002, http://www.ims.nus.edu.sg/Programs/liegroups/abstracts.htm\#lijs2.

49. E. Looijenga, $L^{2}$-cohomology of locally symmetric varieties, Compositio Math. 67 (1988), $3-20$.

50. Y. Matsushima and S. Murakami, On vector bundle valued harmonic forms and automorphic forms on symmetric Riemannian manifolds, Ann. of Math. 78 (1963), 365-416.

51. D. Mauger, Algèbre de Hecke quasi-ordinaire universelle d'un groupe réductif, Thèse de doctorat, Université Paris-Nord, 2000.

52. M. McConnell, Intersection cohomology of toric varieties, preprint, http://www.math.okstate.edu/ mmcconn/toric2.ps, 23 pages, 1999.

53. A. Mokrane and J. Tilouine, Cohomology of Siegel varieties with p-adic integral coefficients and applications, Astérisque (2002), no. 280, 1-95, Cohomology of Siegel varieties.

54. A. Nair, Weighted cohomology of arithmetic groups, Ann. of Math. 150 (1999), 1-31.

55. F. Quinn, Intrinsic skeleta and intersection homology of weakly stratified sets, Geometry and topology (Athens, Ga., 1985), Lecture Notes in Pure and Appl. Math., vol. 105, Dekker, New York, 1987, pp. 233-249.

56. M. S. Raghunathan, Vanishing theorems for cohomology groups associated to discrete subgroups of semisimple Lie groups, Osaka J. Math. 3 (1966), 243-256.

57. _ Corrections to "Vanishing theorems ... ", Osaka J. Math. 16 (1979), 295-299.

58. M. Rapoport, 1986, letter to A. Borel.

59. - On the shape of the contribution of a fixed point on the boundary: The case of $\mathbb{Q}$ rank 1 (with an appendix by L. Saper and M. Stern), The Zeta functions of Picard modular surfaces (R. P. Langlands and D. Ramakrishnan, eds.), Les Publications CRM, Montréal, 1992, pp. 479-488.

60. L. Saper, Tilings and finite energy retractions of locally symmetric spaces, Comment. Math. Helv. 72 (1997), 167-202.

61. L L-modules and micro-support, submitted, arXiv:math.RT/0112251, v2, 88 pages, 2001.

62. L L-modules and the conjecture of Rapoport and Goresky-MacPherson, to appear in Proceedings of the Automorphic Semester at Institute Henri Poincaré, February - June 2000, arXiv:math.RT/0112250, v2, 16 pages, 2001.

63. September 27, 2002, letter to W. Casselman.

64. - Geometric rationality of equal-rank Satake compactifications, submitted, arXiv:math.RT/0211138, 14 pages, 2002.

65. L. Saper and M. Stern, $L_{2}$-cohomology of arithmetic varieties, Ann. of Math. 132 (1990), $1-69$.

66. I. Satake, On a generalization of the notion of a manifold, Proc. Nat. Acad. Sci. U. S. A. 42 (1956), 359-363.

67. On representations and compactifications of symmetric Riemannian spaces, Ann. of Math. 71 (1960), 77-110.

68. - On compactifications of the quotient spaces for arithmetically defined discontinuous groups, Ann. of Math. 72 (1960), 555-580.

69. J. Schwermer, Eisenstein series and cohomology of arithmetic groups: the generic case, Invent. Math. 116 (1994), 481-511.

70. A. Shepard, A cellular description of the derived category of a stratified space, Ph.D. dissertation, Brown University, 1985.

71. G. Shimura, Introduction to the arithmetic theory of automorphic functions, Publications of the Mathematical Society of Japan, vol. 11, Princeton University Press, Princeton, NJ, 1994, Reprint of the 1971 original, Kanô Memorial Lectures, 1.

72. M. Sugiura, Conjugate classes of Cartan subalgebras in real semisimple Lie algebras, J. Math. Soc. Japan 11 (1959), 374-434.

73. Correction to my paper: "Conjugate classes of Cartan subalgebras in real semisimple Lie algebras”, J. Math. Soc. Japan 23 (1971), 379-383. 
74. J. Tilouine and E. Urban, Several variable p-adic families of Siegel-Hilbert cusp eigensystems and their Galois representations, Ann. Sci. École Norm. Sup. (4) 32 (1999), 499-574.

75. J. Tits, Classification of algebraic semisimple groups, Algebraic Groups and Discontinuous Subgroups (Providence), Proc. Symposia Pure Math., vol. 9, Amer. Math. Soc., 1966, pp. 3362.

76. W. T. van Est, A generalization of the Cartan-Leray sequence, I, II, Indag. Math. 20 (1958), 399-413.

77. D. Vogan, Jr. and G. Zuckerman, Unitary representations with nonzero cohomology, Compositio Math. 53 (1984), no. 1, 51-90.

78. M. Vybornov, Constructible sheaves on simplicial complexes and Koszul duality, Math. Res. Lett. 5 (1998), no. 5, 675-683.

79. Sheaves on triangulated spaces and Koszul duality, preprint, arXiv:math.AT/9910150, v2, 41 pages, 2000.

80. J. Wildeshaus, Degeneration of automorphic sheaves, Talk at the Conference "Shimura Varieties and Automorphic Forms", Japan-U.S. Mathematics Institute, Johns Hopkins University, March 2001.

81. S. Zucker, $L_{2}$ cohomology of warped products and arithmetic groups, Invent. Math. 70 (1982), 169-218.

82. _ Satake compactifications, Comment. Math. Helv. 58 (1983), 312-343.

83. _ $L_{2}$-cohomology and intersection homology of locally symmetric varieties, II, Compositio Math. 59 (1986), 339-398.

Department of Mathematics, Duke University, Box 90320, Durham, NC 27708, U.S.A.

E-mail address: saper@math.duke.edu

$U R L:$ http://www.math.duke.edu/faculty/saper 